\title{
SINGULARITY SUBSCHEMES AND GENERIC PROJECTIONS
}

\author{
BY
}

\author{
JOEL ROBERTS $\left({ }^{1}\right)$
}

\begin{abstract}
Corresponding to a morphism $f: V \rightarrow W$ of algebraic varieties (such that $\operatorname{dim}(V) \leqslant \operatorname{dim}(W)$ ), we construct a family of subschemes $s_{1}^{(q)}(f) \subset V$. When $V$ and $W$ are nonsingular, the $S_{1}^{(q)}, q \geqslant 1$, induce a filtration of the set of closed points $x \in V$ such that the tangent space map $d f_{x}: T(V)_{x} \rightarrow T(W)_{f(x)}$ has rank $=\operatorname{dim}(V)-1$. We prove that if $V$ is a suitably embedded nonsingular projective variety and $\pi: V \rightarrow \mathrm{p}^{m}$ is a generic projection, then the $S_{1}^{(q)}(f)$ and certain fibre products of several of the $S_{1}^{(q)}(f)$ are either empty or smooth and of the smallest possible dimension, except in cases where $q+1$ is divisible by the characteristic of the ground field. We apply this result to describe explicitly the ring homomorphisms $\pi^{*}: \hat{0}_{\mathrm{p} m} \pi(x) \rightarrow \hat{0}_{V, x}$ and (when $m \geqslant r+1$ ) to study the local structure of the image $V^{\prime}=\pi(V) \subset \mathrm{P}^{m}$.
\end{abstract}

0 . Introduction. Let $k$ be an algebraically closed field, and let $V$ be a nonsingular projective variety over $k$. As in [11], we consider an embedding $V \subset \mathrm{P}^{n}$ (projective $n$-space) and a finite morphism $\pi: V \rightarrow \mathrm{P}^{m}$, where $\operatorname{dim}(V) \leqslant$ $m \leqslant 2 \cdot \operatorname{dim}(V)$, induced by projection from an $(n-m-1)$-subspace $L \subset \mathbf{P}^{n}$. We study certain properties of generic projections, i.e. properties which $\pi$ has when $L$ corresponds to a point of a suitable dense open subset of the Grassmann variety $G(n, n-m-1)$.

When $m>\operatorname{dim}(V)$, one objective is to describe the structure of the local rings $\hat{O}_{V^{\prime}, y}$, where $y \in V^{\prime}=\pi(V) \subset \mathrm{P}^{m}$. (If $(A, \mathrm{~m})$ is a local ring, then $\hat{A}$ is its completion in the m-adic topology.) Using our main results, which are stated in $\S 1$, this can be done in the case that the map of Zariski tangent spaces $d \pi_{x}: T_{V, x}$ $\rightarrow T_{\mathrm{p} m, y}$ has rank $\geqslant \operatorname{dim}(V)-1$ for all $x \in \pi^{-1}(y)$. Details of this are given in $\S 13$. If $m=\operatorname{dim}(V)$, the corresponding problem is to describe the local structure of the branch locus $\pi\left(S_{1}(\pi)\right) \subset \mathrm{P}^{m}$. $\left(S_{1}(\pi)\right.$ is defined in $\S 2$.) I have obtained some results concerning this problem which will appear in another paper. (See also [14].) The results of the present paper were announced in [13]. I would

Received by the editors July $25,1974$.

AMS (MOS) subject classifications (1970). Primary 14B05, 14E15, $14 \mathrm{NO5}$.

Key words and phrases. Projective algebraic variety, generic projection, Kähler differentials, algebra of principal parts, noetherian scheme, scheme-theoretic fibre, singularity subscheme.

$\left.{ }^{1}\right)$ Supported by NSF grants GP 20550 and GP 37689. 
like to thank the referee for suggesting several ways to improve this paper.

Our techniques involve studying the higher order singularity subschemes of the morphism $\pi: V \rightarrow \mathrm{P}^{m}$. Some properties of these subschemes are described in $\S 1$; the definition is given in $\S 3$.

All rings and algebras will be assumed to be commutative with identity; all schemes and morphisms of schemes will be assumed to be separated. The field $k$ may always be assumed to be algebraically closed, but of arbitrary characteristic. A variety is a reduced and irreducible scheme of finite type over $k$.

1. Statement of the main results. Let $f: X \rightarrow Y$ be a morphism of varieties (over the algebraically closed field $k$ ). In this section we will assume that $X$ is nonsingular. When $i \geqslant 1$, let $S_{i}(f) \subset X$ be the closed subset whose closed points are all $x \in X$ such that the map of Zariski tangent spaces $(d f)_{x}: T(X)_{x} \rightarrow$ $T(Y)_{f(x)}$ has $\operatorname{rank} \leqslant \operatorname{dim}(X)-i$.

If $\operatorname{dim}(X) \leqslant \operatorname{dim}(Y)$, one can study the points where $(d f)_{x}$ has rank $=$ $\operatorname{dim}(X)-1$. To do this, we define closed subschemes $S_{1}^{(q)}(f) \subset X-S_{2}(f)$, for $q \geqslant 0$, called higher order singularity subschemes. (See $\S 3$.) $S_{1}^{(q)}(f)$ is defined in terms of a certain Fitting ideal of the $0_{X}$-algebra $P_{X / Y}^{q}$ of relative principal parts. If $x$ is a closed point of $X-S_{2}(f)$ and $y=f(x)$, then $x \in S_{1}^{(q)}(f)$ if and only if $\operatorname{dim}_{k} O_{x} / \mathrm{m}_{y} \mathrm{O}_{x} \geqslant q+1$, where $\left(\mathrm{O}_{x}, \mathrm{~m}_{x}\right)=\mathrm{O}_{X, x}$ is an algebra over $\left(O_{y}, \mathrm{~m}_{y}\right)=\mathrm{O}_{Y, y}$ via the usual homomorphism $f^{*}: \mathrm{O}_{y} \rightarrow \mathrm{O}_{x}$. The $S_{1}^{(q)}(f)$ are closely related to the Thom-Boardman singularities $S_{1, \ldots, 1}$ ( $q$ indices) of a map of differentiable manifolds. (See [16], [17, §12], [5], [1], or [7] .) ThomBoardman singularities in algebraic geometry have been studied in [6] and [8].

With $f$ as above and $d \geqslant 1$, let $\Sigma_{d}(f) \subset X \times_{Y} \cdots \times_{Y} X$ (d-fold fibre product) be the complement of the union of all diagonals. If $q_{1}, \ldots, q_{d}$ are nonnegative integers, we regard $S_{1}^{\left(q_{1}\right)}(f) \times_{Y} \cdots \times_{Y} S_{1}^{\left(q_{d}\right)}(f)$ as a subscheme of $X \times_{Y} \cdots \times_{Y} X$.

DEFINITION 1.1. $\Sigma_{d}\left(f ; q_{1}, \ldots, q_{d}\right)=\Sigma_{d}(f) \cap\left(S_{1}^{\left(q_{1}\right)}(f) \times_{Y} \cdots \times \times_{Y}\right.$ $\left.S_{1}^{\left(q_{d}\right)}(f)\right)$.

One can regard $\Sigma_{d}\left(f ; q_{1}, \ldots, q_{d}\right)$ as consisting of all $d$-tuples $\left(x_{1}, \ldots, x_{d}\right)$ of distinct closed points of $V$ such that (a) $x_{j} \in S_{1}^{\left(q_{j}\right)}(f), j=1, \ldots, d$, and (b) $f\left(x_{1}\right)=\cdots=f\left(x_{d}\right)$.

THEOREM A. Let $V$ be a nonsingular projective variety over $k$, and let $r \leqslant m \leqslant 2 r$, where $r=\operatorname{dim}(V)$. Then there is an embedding $V \subset \mathbf{P}^{n}$, for some $n$, such that if $\pi: V \rightarrow \mathrm{P}^{m}$ is a generic projection, then the following statements hold for every $d \geqslant 1$ and every d-tuple $\left(q_{1}, \ldots, q_{d}\right)$ of nonnegative integers.

(i) $\Sigma_{d}\left(\pi ; q_{1}, \ldots, q_{d}\right)$ is either empty or of pure dimension $d r-(d-1) m$ $-\sum_{j=1}^{d} q_{j}(m-r+1)$.

(ii) If $\operatorname{char}(k) \nmid\left(q_{j}+1\right)$ for all $j$, then $\Sigma_{d}\left(\pi ; q_{1}, \ldots, q_{d}\right)$ is smooth (over $k$ ). 
(iii) In general, the dimension of the nonsmooth locus of $\Sigma_{d}\left(\pi ; q_{1}, \ldots, q_{d}\right)$ is $\leqslant \operatorname{card}(Q)-1$, where $Q=\left\{j \mid \operatorname{char}(k)\right.$ divides $\left.q_{j}+1\right\}$.

Theorem 10.5 and Corollary 10.6 give a more detailed version of (iii). Nonsmooth points do occur for some generic projections of varieties over fields of positive characteristic. The simplest example is a pinch point of a generic projection $\pi: V^{2} \rightarrow \mathrm{P}^{3}$ when $\operatorname{char}(k)=2$. The results of [12] imply that there exist surfaces whose generic projections actually exhibit pinch points.

Corollary 1.2. Let $\pi: V \rightarrow \mathrm{P}^{m}$ be as in Theorem A.

(i) For every $q \geqslant 0, S_{1}^{(q)}(\pi)$ is either empty or of pure codimension $q(m-r+1)$ in $V$.

(ii) If $\operatorname{char}(k) \nmid(q+1)$, then $S_{1}^{(q)}(\pi)$ is smooth over $k$.

(iii) If $\operatorname{char}(k) \mid(q+1)$, then $S_{1}^{(q)}(\pi)$ is smooth except at finitely many points of $S_{1}^{(q)}(\pi)-S_{1}^{(q+1)}(\pi)$.

When $d r-(d-1) m-\Sigma_{j=1}^{d} q_{j}(m-r+1)<0$, conclusion (i) of Theorem A implies that $\Sigma_{d}\left(\pi ; q_{1}, \ldots, q_{d}\right)=\varnothing$. When $m \geqslant r+1$, this implies that $\Sigma_{d}(\pi)=$ $\varnothing$ when $d>m /(m-r)$. If $m=r$, it also follows that only finitely many $\Sigma_{d}(\pi)$ are nonempty. Specifically, $\Sigma_{d}(\pi)=\varnothing$ when $d>\operatorname{degree}(V)$.

Corollary 1.3. Let $\pi: V \rightarrow \mathrm{P}^{m}$ be as in Theorem A. Then only finitely many of the subschemes $\Sigma_{d}\left(\pi ; q_{1}, \ldots, q_{d}\right)$ are nonempty. If either $\operatorname{char}(k)=0$ or char $(k)>(m+1) /(m-r+1)$, then all nonempty $\Sigma_{d}\left(\pi ; q_{1}, \ldots, q_{d}\right)$ are smooth. (Thus, for a given value of $r=\operatorname{dim}(V)$, nonsmooth $\Sigma_{d}\left(\pi ; q_{1}, \ldots, q_{d}\right)$ will occur only when char $(k)$ assumes finitely many values.)

Proof. We observe that $S_{1}^{(q)}(\pi)=\varnothing$ when $q>r /(m-r+1)$. Therefore, if $\operatorname{char}(k)>1+r /(m-r+1)=(m+1) /(m-r+1)$, then $S_{1}^{(q)}(\pi)=\varnothing$ for all $q$ such that $\operatorname{char}(k) \mid(q+1)$. Q.E.D.

Theorem $A$ is proved in $\S \S 10$ and 11 . Preliminary results needed in the proof are proved in $\S \S 5$ through 9. When $\operatorname{char}(k)=0$, the proof of Theorem $A$ is shorter and more conceptual than in the general case. This is because it is possible to apply Theorem 2.9, which is an algebraic version of Sard's Theorem. The proof of the characteristic 0 case of Theorem A uses nothing from $\S \S 7$ and 11.

THEOREM B. Let $f: X \rightarrow Y$ and $f^{\prime}: X^{\prime} \rightarrow Y^{\prime}$ be finite morphisms of nonsingular varieties over $k$, where $\operatorname{dim}\left(X^{\prime}\right)=\operatorname{dim}(X) \leqslant \operatorname{dim}(Y)=\operatorname{dim}\left(Y^{\prime}\right)$. Let $q_{1}, \ldots, q_{d}$ be nonnegative integers, and let $x_{1}, \ldots, x_{d}$ and $x_{1}^{\prime}, \ldots, x_{d}^{\prime}$ be distinct closed points of $X$ and $X^{\prime}$ respectively. Assume $\ldots, d$;

(a) $x_{j} \in S_{1}^{\left(q_{j}\right)}(f)-S_{1}^{\left(q_{j}+1\right)}(f)$ and $x_{j}^{\prime} \in S_{1}^{\left(q_{j}\right)}\left(f^{\prime}\right)-S_{1}^{\left(q_{j}+1\right)}\left(f^{\prime}\right), j=1$, 
(b) $S_{1}^{\left(q_{1}\right)}(f) \times_{Y} \cdots \times_{Y} S_{1}^{\left(q_{d}\right)}(f)$ and $S_{1}^{\left(q_{1}\right)}\left(f^{\prime}\right) \times_{Y^{\prime}} \cdots \times_{Y^{\prime}} S_{1}^{\left(q_{d}\right)}\left(f^{\prime}\right)$ are smooth and of pure dimension $d r-(d-1) m-\Sigma_{j=1}^{d} q_{j}(m-r+1)$ at $\left(x_{1}, \ldots, x_{d}\right)$ and $\left(x_{1}^{\prime}, \ldots, x_{d}^{\prime}\right)$ respectively.

Then there are isomorphisms $\psi: \hat{O}_{Y, y} \approx \hat{O}_{Y^{\prime}, y^{\prime}}$ and $\phi_{j}: \hat{O}_{X, x_{j}} \approx \hat{O}_{X^{\prime}, x_{j}^{\prime}}$, $j=1, \ldots, d$, such that $f_{j}^{*}=\phi_{j} \circ f_{j}^{*} \circ \psi^{-1}$ for $j=1, \ldots, d$, where $f_{j}^{*}: \hat{O}_{Y, y}$ $\rightarrow \hat{O}_{X, x_{j}}$ is induced by $f$ and $f_{j}^{\prime *}$ is similarly induced by $f^{\prime}$.

In the case that $\left\{x_{1}, \ldots, x_{d}\right\}=f^{-1}(y)$, one can consider the scheme theoretic fibre $\operatorname{Spec}\left(A_{y}\right)$, where $A_{y}=0_{X, f^{-1}(y)} / f^{*}\left(\mathrm{~m}_{y}\right) \cdot O_{X, f^{-1}(y)}$. (The homomorphism $f^{*}: O_{y} \rightarrow O_{X, f^{-1}(y)}$ is induced by $f$. Note that $A_{y}$ is an Artinian ring.) The conclusion of Theorem $B$ can be interpreted as saying that the homomorphism

$$
f^{*}: \hat{O}_{Y, y} \rightarrow \hat{O}_{X, f^{-1}(y)}=\prod_{j=1}^{d} \hat{O}_{X, x_{j}}
$$

depends only on $A_{y}$, up to automorphisms of the domain and range of $f^{*}$.

Theorem $B$ is proved in $\S 12$. Specifically, Theorem 12.1 shows how to identify $\hat{\theta}_{Y, y}$ and $\hat{\theta}_{X, x_{j}}, j=1, \ldots, d$, with formal power series rings in a way which makes it possible to give a very explicit description of $f^{*}$. We call this description a canonical form of $f^{*}$.

Theorem $B$ is relevant to the study of generic projections because a generic projection $\pi: V \rightarrow \mathrm{P}^{m}$ satisfies the hypotheses of Theorem B under the circumstances described by Theorem $A$.

REMARK 1.4. Theorem 3 of [13] contains a statement which would seem to imply that if $\pi: V^{r} \rightarrow \mathrm{P}^{m}$ is a generic projection and $d \geqslant 2$, then all nonempty $\Sigma_{d}\left(\pi ; q_{1}, \ldots, q_{d}\right)$ are smooth. However, this is not the case; counterexamples can be given with $r=m=2$ and $\operatorname{char}(k)=2$.

2. First-order singularity subschemes. In this section we define and study subschemes $S_{i}(f) \subset X$ corresponding to certain morphisms $f: X \rightarrow Y$. The definition is given in terms of Fitting ideals; we begin by recalling the definition of these ideals.

Let $A$ be a ring, $M$ a finitely presented $A$-module. Consider a presentation:

$$
A^{m} \stackrel{\psi}{\longrightarrow} A^{r} \rightarrow M \rightarrow 0,
$$

where $\psi\left(e_{j}\right)=\Sigma_{k=1}^{r} a_{j k} f_{k}$ relative to standard bases $\left\{e_{1}, \ldots, e_{m}\right\}$ and $\left\{f_{1}, \ldots, f_{r}\right\}$. The ith Fitting ideal $\Delta_{i}(M) \subset A$ is defined to be the ideal generated by the $(r-i) \times(r-i)$ minors of the matrix $\left(a_{j k}\right)$ [resp. the unit ideal] if $0 \leqslant i$ $<r$ [resp. $i \geqslant r$ ]. It is known (see [3]) that the $\Delta_{i}(M)$ are independent of the choice of the presentation $(*)$. We note that $\Delta_{i}(M) \subset \Delta_{i+1}(M)$ for all $i$. Verification of the following lemma is also straightforward. 
LEMMA 2.1. If $(A, \mathrm{~m})$ is local and a minimal generating set for $M$ contains $r$ elements, then $\Delta_{r-1}(M) \neq A$, and $\Delta_{i}(M) \subset \mathrm{m}^{r-i}$ when $0 \leqslant i \leqslant r-1$.

Since the Fitting ideals of a module are independent of the presentation used to compute them, we can define Fitting ideals of coherent sheaves on a Noetherian scheme.

Proposition 2.2. Let $X$ be a Noetherian scheme, $F$ a coherent sheaf of modules on $X$. For each $i \geqslant 0$ there is a unique sheaf of ideals $\Delta_{i}(F) \subset O_{X}$ such that $\Gamma\left(U, \Delta_{i}(F)\right)=\Delta_{i}(\Gamma(U, F))$ for every affine open $U \subset X$. If $x \in X$, then $\Delta_{i}(F)_{x}=\Delta_{i}\left(F_{x}\right)$.

We call $\Delta_{i}(F)$ the ith Fitting ideal of $F$.

We now fix a morphism $f: X \rightarrow Y$ of Noetherian schemes. It will be assumed that the sheaf $\Omega_{X / Y}^{1}$ of relative Kähler differentials is coherent.

Definition 2.3. Let $i \geqslant 1 . S_{i}(f) \subset X$ is the closed subscheme defined by $\Delta_{i-1}\left(\Omega_{X / Y}^{1}\right)$. We call the $S_{i}(f)$ first-order singularity subschemes.

We write $S_{i}$ for $S_{i}(f)$ when the context is clear. By using properties of Fitting ideals, one sees that $S_{i} \supset S_{i+1}$.

Proposition 2.4. If $x \in X$, then $x \in S_{i}$ if and only if $\operatorname{dim}_{k(x)} \Omega_{X / Y}^{1}(x) \geqslant$ $i$. Equality holds when $x \in S_{i}-S_{i+1}$. If $X$ and $Y$ are varieties over an algebraically closed field $k$, and $x$ is a closed point, then $x \in S_{i}$ if and only if the map of Zariski tangent spaces $d f_{x}: T_{X, x} \rightarrow T_{Y, f(x)}$ has rank $\leqslant r-i$, where $r=$ $\operatorname{dim}_{k}\left(T_{X, x}\right)$.

Proposition 2.5. $S_{i+1} \subset \operatorname{Sing}\left(S_{i}\right)$.

Proof. To establish either result, we apply Lemma 2.1 with $(A, \mathrm{~m})=0_{X, x}$ and $M=\left(\Omega_{X / Y}^{1}\right)_{x}$. In particular, if $j>i$ and $x \in S_{j}-S_{j+1}$, then $\Delta_{i-1}(M) \subset$ $\mathbf{m}^{j-i+1}$. By considering the exact sequence of vector spaces

$$
\left(f^{*} \Omega_{Y / k}^{1}\right)(x) \rightarrow \Omega_{X / k}^{1}(x) \rightarrow \Omega_{X / Y}^{1}(x) \rightarrow 0,
$$

one proves the last statement of Proposition 2.4. Q.E.D.

Proposition 2.6. Let $g: X \rightarrow Z$ and $h: Y \rightarrow Z$ be morphisms of Noetherian schemes such that $g=h \circ f$. Let $f^{\prime}: X^{\prime} \rightarrow Y^{\prime}$ be obtained by base extension, where $X^{\prime}=X \times{ }_{Z} Z^{\prime}$ and $Y^{\prime}=Y \times{ }_{Z} Z^{\prime}$. ( $Z^{\prime}$ is a scheme over $Z$.) Then $S_{i}\left(f^{\prime}\right)=S_{i}(f) \times{ }_{Z} Z^{\prime}$ for all $i$.

Proof. It is well known that $\Omega_{X^{\prime} / Y^{\prime}}^{1}=v^{*}\left(\Omega_{X / Y}^{1}\right)$, where $v: X^{\prime} \rightarrow X$ is projection to the first factor. We now obtain the conclusion by applying the following lemma.

Lemma 2.7. Let $v: X^{\prime} \rightarrow X$ be a morphism of Noetherian schemes, and 
let $E$ be a coherent sheaf on $X$. Then $\Delta_{i}\left(v^{*} E\right)=v^{*}\left(\Delta_{i}(E)\right)$ for all $i$.

Proof. After reducing to the case where $X=\operatorname{Spec}(A), X^{\prime}=\operatorname{Spec}(B)$ and $E=\widetilde{M}$, we apply $\bigotimes_{A} B$ to the exact sequence (*). Q.E.D.

Proposition 2.8. Let $x \in X, y=f(x) \in Y$. Let $g, h$ be as in Proposition 2.6, and assume that $\left(\Omega_{X / Z}^{1}\right)_{x}$ and $\left(\Omega_{Y / Z}^{1}\right)_{y}$ are free modules of rank $r$ and $m$ respectively. If either $m \geqslant r$ or $r-i \leqslant m \leqslant r$ holds, then every component of $S_{i}(f)$ which contains $x$ has codimension (in $\left.X\right) \leqslant i(m-r+i)$.

Proof. Let $z=g(x)=h(y) \in Z$ and set $A=O_{Z, z}, B=O_{Y, y}$, and $C=$ $O_{X, x}$. We have an exact sequence of $C$-modules:

$$
\Omega_{B / A}^{1} \otimes_{B} C \rightarrow \Omega_{C / A}^{1} \rightarrow \Omega_{C / B}^{1} \rightarrow 0 .
$$

The first two terms are free of rank $m$ and $r$ respectively, so that $\Delta_{i-1}\left(\Omega_{X / Y}^{1}\right)_{x}=$ $\Delta_{i-1}\left(\Omega_{C / B}^{1}\right)$ is generated by the $(r-i+1) \times(r-i+1)$ minors of an $m \times r$ matrix. By Theorem 3 of Eagon and Northcott [2], every minimal prime ideal of $\Delta_{i-1}\left(\Omega_{C / B}^{1}\right)$ has height $\leqslant i(m-r+i)$. Q.E.D.

The following result is probably well known, but I have included the proof because it is a direct application of the concepts introduced above.

THEOREM 2.9. Let $f: X^{r} \rightarrow Y^{m}$ be a morphism of nonsingular varieties over an algebraically closed field of characteristic 0 . If $r \geqslant m$, then either:

$(\alpha) f(X)$ is contained in a proper closed subset of $Y$, or

$(\beta)$ there is a dense open subset of $Y$ consisting of closed points $y$ such that $f^{-1}(y)$ is smooth, nonempty, and of pure dimension $r-m$.

Proof. If $x$ is a closed point of $X$, then every component of $f^{-1}(f(x))$ has dimension $\geqslant r-m$, and the Zariski tangent space $T_{f^{-1}(f(x)), x}$ is isomorphic to the kernel of the linear map $d f_{x}: T_{X, x} \rightarrow T_{Y, f(x)}$. Therefore, $f^{-1}(f(x))$ is smooth and of dimension $r-m$ at $x$ if and only if $x \notin S_{r-m+1}(f)$.

Let $Z$ be an irreducible component of $S_{r-m+1}(f)$. It must be shown that $\operatorname{dim} \overline{(f(Z))}<\operatorname{dim}(Y)$. Assume that this is false, so that $g=\left.f\right|_{Z}$ is dominating. $T_{Z, x} \subset T_{X, x}$ for all closed points $x \in X$. Therefore, $d g_{x}: T_{Z, x} \rightarrow T_{Y, g(x)}$ has rank $\leqslant m-1$ for every closed point $x \in X$, so that $\operatorname{dim}_{k(x)} \Omega_{Z / Y}^{1}(x) \geqslant n-m+1$ for every point $x$ (closed or not) of $Z$, where $n=\operatorname{dim}(Z)$. Taking $x$ to be the generic point of $Z$, we obtain

$$
\operatorname{dim}_{k(Z)} \Omega_{k(Z) / k(Y)}^{1}>\operatorname{dim}(Z)-\operatorname{dim}(Y)=\operatorname{tr} \operatorname{deg}_{k(Y)} k(Z) .
$$

(Note that $k(Y) \subset k(Z)$ since $g: Z \rightarrow Y$ is dominating.) We have thus obtained a contradiction. (Since $\operatorname{char}(k)=0$, the field extension must be separable.) Q.E.D.

EXAMPLE 2.10. Let char $(k)=2$, and let $f: \mathbf{A}^{2} \rightarrow \mathbf{A}^{1}$ send $\left(a_{1}, a_{2}\right) \rightarrow$ 
$a_{1}^{2}+a_{2}^{3}$. For each $a \in k, f^{-1}(a)$ is the curve $\left(X_{1}+\alpha\right)^{2}+X_{2}^{3}=0$, where $\alpha^{2}=$ a. Each of these curves has a cusp, so that the conclusion of Theorem 2.9 fails. We note that $S_{2}(f)$ is the line $X_{2}=0$. Therefore, $g=\left.f\right|_{S_{2}(f)}: S_{2}(f) \rightarrow \mathrm{A}^{1}$ is surjective and induces a purely inseparable field extension of degree 2.

3. Higher order singularity subschemes. We again fix a morphism $f: X \rightarrow$ $Y$ of Noetherian schemes such that $\Omega_{X / Y}^{1}$ is coherent. Let $q \geqslant 0$, and let $P_{X / Y}^{q}$ be the $O_{X}$-algebra of $q$ th order relative principal parts corresponding to $f$. We recall that $P_{X / Y}^{q}=p_{1 *}\left(O_{S} / J^{q+1}\right)$, where $S=X \times_{Y} X, J \subset O_{S}$ is the sheaf of ideals defining the diagonal, and $p_{1}: S \rightarrow X$ is the projection to the first factor. The $O_{X}$-algebra structure $O_{X} \rightarrow P_{X / Y}^{q}$ is deduced from $p_{1}$. As an $O_{X}$-module, $P_{X / Y}^{q}$ has a finite filtration with factors $p_{1 *}\left(J^{i} / J^{i+1}\right)$. Since $p_{1 *}\left(J / J^{2}\right)=\Omega_{X / Y}^{1}$, $P_{X / Y}^{q}$ is coherent.

DEFINITION 3.1. Let $q \geqslant 0$. We define the qth order singularity subscheme $S_{1}^{(q)}(f) \subset X$ to be the closed subscheme of $X-S_{2}(f)$ corresponding to the sheaf of ideals $\Delta_{q}\left(P_{X / Y}^{q}\right) \subset O_{X}$.

Since $P_{X / Y}^{0}=0_{X}$ and $P_{X / Y}^{1} \cong 0_{X} \oplus \Omega_{X / Y}^{1}$, we have $S_{1}^{(0)}(f)=X-S_{2}(f)$ and $S_{1}^{(1)}(f)=S_{1}(f)-S_{2}(f)$. The geometric significance of the remaining $S_{1}^{(q)}(f)$ will be explained by Proposition 3.5 below.

Proposition 3.2. Assume that $x \in X-S_{2}(f)$. Then $x \in S_{1}^{(q)}(f)$ if and only if $\operatorname{dim}_{k(x)} P_{X / Y}^{q}(x) \geqslant q+1$. In particular $S_{1}^{(q)}(f) \supset S_{1}^{(q+1)}(f)$.

Proof. Apply Lemma 2.1 with $A=0_{X, x}$ and $M=\left(P_{X / Y}^{q}\right)_{x}$. The last statement follows because there is a surjection $P_{X / Y}^{q+1} \rightarrow P_{X / Y}^{q}$. Q.E.D.

Proposition 3.3. Let $g: X \rightarrow Z$ and $h: Y \rightarrow Z$ be morphisms of Noetherian schemes such that $g=h \circ f$. Let $u: Z^{\prime} \rightarrow Z$ be a morphism, and let $f^{\prime}: X^{\prime}$ $\rightarrow Y^{\prime}$ be obtained by base extension, where $X^{\prime}=X \times_{Z} Z^{\prime}$ and $Y^{\prime}=Y \times_{Z} Z^{\prime}$. Then $S_{1}^{(q)}\left(f^{\prime}\right)=S_{1}^{(q)}(f) \times{ }_{Z} Z^{\prime}$ for all $q$.

Proof. By Proposition 16.4.5 of [4], $P_{X^{\prime} / Y^{\prime}}^{q}=v^{*}\left(P_{X / Y}^{q}\right)$, where $v: X^{\prime}=$ $X \times{ }_{Z} Z^{\prime} \rightarrow X$ is projection to the first factor. One completes the proof by applying Lemma 2.7 . Q.E.D.

If $A$ is a ring and $B$ is an $A$-algebra, then $P_{\operatorname{Spec}(B) / \operatorname{Spec}(A)}^{q}$ is the sheaf on $\operatorname{Spec}(B)$ corresponding to the $B$-algebra $P_{B / A}^{q}=\left(B \otimes_{A} B\right) / I^{q+1}$, where $I$ is the kernel of the multiplication map $\mu: B \otimes_{A} B \rightarrow B$. The $B$-algebra structure is given by $j: B \rightarrow P_{B / A}^{q}$, where $j(b)=\overline{b \otimes 1}$. Another interesting homomorphism is $d^{q}=d_{B / A}^{q}: B \rightarrow P_{B / A}^{q}$, where $d^{q}(b)=\overline{1 \otimes b}$.

LEMMA 3.4. Let $k$ be a field, $\left(A, \mathrm{~m}_{A}\right)$ and $\left(B, \mathrm{~m}_{B}\right)$ local rings with residue fields $\cong k$, and $g: A \rightarrow B$ a local homomorphism which induces an isomorphism of residue fields. There there is a k-algebra isomorphism $\phi: k \otimes_{B} P_{B / A}^{q} \stackrel{\approx}{\longrightarrow}$ 
$B /\left(g\left(\mathrm{~m}_{A}\right) B+\mathrm{m}_{B}^{q+1}\right)$ such that $\phi\left(1 \otimes d^{q} b\right)=\bar{b}$ (residue class of $\left.b\right)$ for all $b \in B$.

Proof. Associativity of the tensor product implies that $k \otimes_{B}\left(B \otimes_{A} B\right) \cong$ $B / g\left(\mathrm{~m}_{A}\right) B$. (Observe that $k \otimes_{A} B=B / g\left(\mathrm{~m}_{A}\right) B$.) Thus, the residue class map $B / g\left(\mathrm{~m}_{A}\right) B \rightarrow k$ is identified with id $\log \otimes: k \otimes_{B}\left(B \otimes_{A} B\right) \rightarrow k \otimes_{B} B=k$. Therefore,

$$
\left[k \otimes_{B}\left(B \otimes_{A} B\right)\right] /\left(\operatorname{Ker}\left(\mathrm{id}_{k} \otimes \mu\right)\right)^{q+1} \cong B /\left(g\left(\mathrm{~m}_{A}\right) B+\mathrm{m}_{B}^{q+1}\right) .
$$

It is easily shown that this implies the desired conclusion. Q.E.D.

Proposition 3.5. Let $q \geqslant 1$, and assume that $X$ and $Y$ are varieties over the algebraically closed field $k$. Let $x$ be a closed point of $X$. Then $x \in S_{1}^{(q)}(f)$ if and only if (i) $\operatorname{dim}_{k} \mathrm{~m}_{x} /\left(f^{*}\left(\mathrm{~m}_{y}\right) \mathrm{O}_{x}+\mathrm{m}_{x}^{2}\right)=1$ and (ii) $\operatorname{dim}_{k} O_{x} / f^{*}\left(\mathrm{~m}_{y}\right) O_{x} \geqslant$ $q+1$, where $\left(0_{x}, \mathrm{~m}_{x}\right)=0_{X, x}, y=f(x),\left(0_{y}, \mathrm{~m}_{y}\right)=0_{Y, y}$, and $f^{*}: O_{y} \rightarrow O_{x}$ is induced by $f$.

Proof. By Proposition 2.4, (i) holds if and only if $x \in S_{1}-S_{2}=S_{1}^{(1)}$, so we assume that (i) holds. Thus, the maximal ideal of $O_{x} / f^{*}\left(\mathrm{~m}_{y}\right) \mathrm{O}_{x}$ is principal, so that (ii) holds if and only if $\operatorname{dim}_{k} O_{x} /\left(f^{*}\left(\mathrm{~m}_{y}\right) O_{x}+\mathrm{m}_{x}^{q+1}\right) \geqslant q+1$. By Lemma 3.4 and Proposition 3.2, this holds if and only if $x \in S_{1}^{(q)}(f)$. (Note that $\left(P q_{X / Y}\right)_{x}=P_{Q_{x} / O_{y}}$; see [4, Corollaire 16.4.16] or [15, Proposition A 1.2].)

REMARK 3.6. If $X$ and $Y$ are varieties over $k$, then the higher order singularity subschemes are interesting only in the case that $\operatorname{dim}(X) \leqslant \operatorname{dim}(Y)$. Specifically, if $\operatorname{dim}(Y) \leqslant \operatorname{dim}(X)-2$, then $X-S_{2}(f)=\varnothing$. If $\operatorname{dim}(Y)=\operatorname{dim}(X)$ -1 , then (i) and (ii) hold at every closed point $x \in X-S_{2}(f)$ for every $q \geqslant 0$.

If $B$ is a $k$-algebra, then a $k$-linear map $D: B \rightarrow B$ is a differential operator if it is of the form $D=\phi^{\circ} d_{B / k}^{q}$ for some $q \geqslant 0$, where $\phi: p_{B / k}^{q} \rightarrow B$ is a $B$ module homomorphism. The smallest $q$ for which such a factorization exists is the order of $D$.

LEMMA 3.7. Let $X$ be a nonsingular variety over $k, x \in X$ a closed point, and $\left(O_{x}, \mathrm{~m}_{x}\right)=O_{X, x}$. Let $\left\{t_{1}, \ldots, t_{r}\right\} \subset \mathrm{m}_{x}$ be a minimal generating set. Then

(i) $P_{X / \operatorname{Spec}(k)}^{q}$ is a locally free sheaf;

(ii) $\left(P_{X / \operatorname{Spec}(k)}^{q}\right)_{x}=P q_{x} / k$ is freely generated by the monomials of degree $\leqslant q$ in $\zeta_{1}, \ldots, \zeta_{r}$, where $\zeta_{i}=d q_{x} / k t_{i}-t_{i}$;

(iii) corresponding to the r-tuples $\left(i_{1}, \ldots, i_{r}\right)$ of nonnegative integers such that $0 \leqslant i_{1}+\cdots+i_{r} \leqslant q$ there are differential operators $D_{i_{1} \cdots i_{r}}: O_{x} \rightarrow 0_{x}$ of order $\leqslant q$ such that

$$
\begin{gathered}
d g_{x} / k=\sum_{i_{1}, \cdots, i_{r}}\left(D_{i_{1} \cdots i_{r}} b\right) \zeta_{1}^{i_{1}} \cdots \zeta_{r}^{i_{r}} \text { for all } b \in 0_{x}, \\
D_{i_{1} \cdots i_{r}}\left(t_{1}^{n_{1}} \cdots t_{r}^{n_{r}}\right)=\left(\begin{array}{c}
n_{1} \\
i_{1}
\end{array}\right) \cdots\left(\begin{array}{l}
n_{r} \\
i_{r}
\end{array}\right) t_{1}^{n_{1}-i_{1}} \cdots t_{r}^{n_{r}-i_{r}},
\end{gathered}
$$


whenever $n_{\nu} \geqslant 0$ for all $\nu$, subject to the convention that $\left(\begin{array}{l}n \\ i\end{array}\right)=0$ when $n<i$;

(c) more generally, if $N \geqslant q$ and $b \equiv t_{1}^{n_{1}} \cdots t_{r}^{n_{r}}\left(\bmod \mathrm{m}_{x}^{N}\right)$, then

$$
D_{i_{1} \cdots i_{r}} b \equiv\left(\begin{array}{l}
n_{1} \\
i_{1}
\end{array}\right) \cdots\left(\begin{array}{l}
n_{r} \\
i_{r}
\end{array}\right) t_{1}^{n_{1}-i_{1}} \cdots t_{r}^{n_{r}-i_{r}}\left(\bmod \mathrm{m}_{x}^{N-q}\right) .
$$

(We identify $b \in O_{x}$ with its image under $d^{q} o_{x} / k: O_{x} \rightarrow P^{q_{O_{x} / k}}$; thus $d^{q} 0_{x} / k b-b$ is represented by $1 \otimes b-b \otimes 1 \in 0_{x} \otimes_{k} O_{x}$.) Proposition 17.12.4 of [4, Chapitre IV] implies that $P_{X / \operatorname{Spec}(k)}^{q}$ is locally free. (More generally, $P_{X / Z}^{q}$ is locally free if $f: X \rightarrow Z$ is a smooth morphism.) Alternately, Lemma 3.4 can be used to show that $\operatorname{dim}_{k(x)} P_{X / \operatorname{Spec}(k)}^{q}(x)$ is constant. Statements (ii), (iii)(a) and (iii)(b) are immediate consequences of (i), Lemma 3.4, and Nakayama's lemma. (See also [4, Chapitre IV, Théorème 16.11.2].) To establish (iii)(c) we observe that if $D: B \rightarrow B$ has order $\leqslant q$ and $I$ is an ideal in $B$, then $D I^{n} \subset I^{n-q}$ for all $n \geqslant q$. (See [4, Section 16.8] or [15, Proposition A 1.1].)

LEMMA 3.8. Let $f: X^{r} \rightarrow Y^{m} \quad(r \leqslant m)$ be a morphism of nonsingular varieties over $k$, and let the remaining hypotheses and notation be as in Proposition 3.5. Let $\left\{s_{1}, \ldots, s_{m}\right\} \subset \mathrm{m}_{y}$ and $\left\{t_{1}, \ldots, t_{r}\right\} \subset \mathrm{m}_{x}$ be minimal generating sets such that $f^{*}\left(s_{i}\right)=t_{i}$ for $i=1, \ldots, r-1$. Then the ideal $\Delta_{q}\left(P_{X / Y}^{q}\right)_{x}=$ $\Delta_{q}\left(P^{q} O_{x} O_{y}\right) \subset O_{x}$ is generated by the $q(m-r+1)$ elements $D^{(i)} f^{*}\left(s_{l}\right), 1 \leqslant i \leqslant$ $q$ and $r \leqslant l \leqslant m$, where $D^{(i)}: O_{x} \rightarrow O_{x}$ is the differential operator $D_{0} \cdots 0 i$ described in Lemma 3.7.

Using this result and the Krull altitude theorem [9, Theorem 9.3], we obtain

COROLlaRY 3.9. If $f: X^{r} \rightarrow Y^{m}$ is as above, then every irreducible component of $S_{1}^{(q)}(f)$ has dimension $\geqslant r-q(m-r+1)$, or else $S_{1}^{(q)}(f)=\varnothing$.

Proof of Lemma 3.8. By Proposition 16.4.18 of [4], there is an $O_{x}$ algebra homomorphism $w: P q_{x} / k \rightarrow P q_{x} / 0_{y}$ such that the ideal $\operatorname{Ker}(w) \subset P q_{x} / k$ is generated by $\left\{d^{q} f^{*}(a)-f^{*}(a) \mid a \in O_{y}\right\}$, where $d^{q}=d^{q} 0_{x} / k$. If $\zeta_{1}, \ldots, \zeta_{r}$ are as in Lemma 3.7, then $P q_{x} / k=E \oplus F$, where $F$ is freely generated by $1, \zeta_{r}$, $\ldots, \zeta_{r}^{q}$, and $E$ is freely generated by the monomials in $\zeta_{1}, \ldots, \zeta_{r}$ which are not powers of $\zeta_{r}$. Since $\zeta_{i}=d^{q} f^{*}\left(s_{i}\right)-f^{*}\left(s_{i}\right)$ for $i=1, \ldots, r-1$, it follows that $E \subset \operatorname{Ker}(w)$, and $w$ induces a surjection $\bar{w}: F \rightarrow P_{O_{x} / O_{y}}$ of $O_{x}$-modules. Suppose now that $\eta_{1}, \ldots, \eta_{N}$ generate $\operatorname{Ker}(\bar{w})$, where $\eta_{j}=\Sigma_{i=0}^{q} \beta_{j i} \zeta_{r}^{i}$. Then $\left\{\beta_{j i}\right\}$ will generate $\Delta_{q}\left(P q_{x} / 0_{y}\right)$. (Observe that $\operatorname{rank}(F)=\operatorname{dim}_{k}\left(k \otimes_{0_{x}} P q_{x} / 0_{y}\right)$.) Therefore, we need to find a nice set of generators for $\operatorname{Ker}(\bar{w})$.

By Lemma 3.7, $\operatorname{Ker}(w)$ is generated as $O_{x}$-module by the elements $\left(d^{q} f^{*}(a)-f^{*}(a)\right) \zeta_{1}^{i_{1}} \cdots \zeta_{r}^{i_{r}}$, where $a \in 0_{y}$ and $0 \leqslant i_{1}+\cdots+i_{r} \leqslant q$. It is not hard to see that we only need to let $a$ run through the monomials of degree $\leqslant q$ in $s_{1}, \ldots, s_{m}$. To calculate $\Delta_{q}\left(P^{q} q_{x} / 0_{y}\right)$, we must therefore expand the 
elements $d^{q} f^{*}(a)-f^{*}(a)$ as polynomials in $\zeta_{1}, \ldots, \zeta_{r}$ and extract the coefficients of $1, \zeta_{r}, \ldots, \zeta_{r}^{q}$. Since $d^{q}$ is a ring homomorphism, monomials in $s_{1}, \ldots, s_{m}$ of degree $\geqslant 2$ will give rise to coefficients which are polynomials in the coefficients occurring in the expansions of $d^{q} f^{*}\left(s_{l}\right)-f^{*}\left(s_{l}\right), l=r, \ldots, m$. Now

$$
d^{q} f^{*}\left(s_{l}\right)-f^{*}\left(s_{l}\right)=\sum_{i=1}^{q} D^{(i)} f^{*}\left(s_{l}\right) \zeta_{r}^{i}+(\text { other terms of degree } \geqslant 1) .
$$

We conclude that $\left\{D^{(i)} f^{*}\left(s_{l}\right) \mid 1 \leqslant i \leqslant q\right.$ and $\left.r \leqslant l \leqslant m\right\}$ generates $\Delta_{q}\left(P q_{x} / 0_{y}\right)$. Q.E.D.

We now consider certain morphisms of product varieties. Specifically, let $X, Y$, and $Z$ be nonsingular varieties over $k$, and let $f: X \times_{k} Z \rightarrow Y \times_{k} Z$ be a morphism such that $g=h \circ f$, where $g$ and $h$ are the projections of $X \times{ }_{k} Z$ and $Y \times{ }_{k} Z$ to $Z$. Let $(x, z)$ be a closed point of $X \times_{k} Z$, and let $(y, z)=f(x, z)$. Let $\left(R, \mathrm{~m}_{R}\right),\left(A, \mathrm{~m}_{A}\right)$ and $\left(B, \mathrm{~m}_{B}\right)$ be the local rings $O_{Z, z}, O_{Y \times Z,(y, z)}$ and $O_{X \times Z,(x, z)}$ respectively. Then $f^{*}: A \rightarrow B$ is an $R$-algebra homomorphism. In the following proposition, we regard $O_{y}$ and $O_{x}$ as subrings of $A$ and $B$ respectively.

Proposition 3.10. With the notation and assumptions as above, let $\left\{s_{1}, \ldots, s_{m}\right\} \subset \mathrm{m}_{y} A$ and $\left\{t_{1}, \ldots, t_{r}\right\} \subset \mathrm{m}_{x} B$ be minimal generating sets such that $f^{*}\left(s_{j}\right)=t_{j}$ for $j=1, \ldots, r-1$. If $q \geqslant 0$, then there exist differential operators $D^{(l)}: B \rightarrow B, l=1, \ldots, q$, which are also $R$-module homomorphisms, such that

(i) if $b \equiv t_{1}^{i_{1}} \cdots t_{r}^{i_{r}}\left(\bmod \mathrm{m}_{x}^{q+1} B\right)$, then $D^{(l)} b \equiv\left({ }_{l}^{i_{r}}\right) t_{1}^{i_{1}} \cdots t_{r}^{i_{r}-l}$ $\left(\bmod \mathrm{m}_{x}^{q+1-l} B\right)$;

(ii) $\Delta^{q}\left(P_{B / A}^{q}\right)$ is generated by the $q(m-r+1)$ elements $D^{(l)} f^{*}\left(s_{j}\right), \quad 1 \leqslant l$ $\leqslant q$ and $r \leqslant j \leqslant m$.

Proof. Since $P_{B / R}^{q}=\left(P_{X \times Z / Z}^{q}\right)_{(x, z)}, P_{B / R}^{q}$ is a free $B$-module. (The projection to the second factor is a smooth morphism.) We proceed as in the proof of Lemma 3.7 to establish the existence of the differential operators $D^{(l)}: B \rightarrow B$ which satisfy (i) and are also $R$-linear. (In fact, $d_{B / R}^{q}: B \rightarrow P_{B / R}^{q}$ is an $R$-algebra homomorphism.)

In order to show that $\Delta^{q}\left(P_{B / A}^{q}\right)$ has the desired generating set, we represent $P_{B / A}^{q}$ as a homomorphic image of $P_{B / R}^{q}$ and proceed as in the proof of Lemma 3.8. Q.E.D.

4. Consequences of smoothness. The main result of this section is the following theorem, which can be shown to be equivalent to the case $d=1$ of Theorem B.

THEOREM 4.1. Let $f: V^{r} \rightarrow W^{m}$ be a finite morphism of nonsingular varieties over $k$, where $m \geqslant r$. Let $x \in S_{1}^{(q)}(f)-S_{1}^{(q+1)}(f)$, and assume that $S_{1}^{(q)}(f)$ is smooth at $x$ and locally of codimension $q(m-r+1)$ in $V$. Then 
there are isomorphisms $\phi: \hat{0}_{V, x} \stackrel{\approx}{\longrightarrow} B=k\left[\left[t_{1}, \ldots, t_{r}\right]\right]$ and $\psi: \hat{O}_{W, f(x)} \approx$ $A=k\left[\left[s_{1}, \ldots, s_{m}\right]\right]$ such that $g=\phi \circ f^{*} \circ \psi^{-1}: A \rightarrow B$ satisfies

(i) $g\left(s_{i}\right)=t_{i}, i=1, \ldots, r-1$;

(ii)(a) $g\left(s_{r}\right)=t_{r}^{q+1}+\Sigma_{j=1}^{q-1} t_{q(m-r)+j} t_{r}^{j}$ if $\operatorname{char}(k) \nmid(q+1)$;

(ii)(b) $g\left(s_{r}\right)=t_{r}^{q+1}+\Sigma_{j=1}^{q} t_{q(m-r)+t_{r}^{t}}^{t}$ if $\operatorname{char}(k) \mid(q+1)$, and $q(m-r+1)$

$<r$ in this case;

(iii) $g\left(s_{r+i}\right)=\Sigma_{j=1}^{q} t_{q(i-1)+j} t_{r}^{j}, i=1, \ldots, m-r$.

Before giving the proof, we will discuss certain algebras of principal parts.

Lemma 4.2 [4, Proposition 16.4.20]. Let $A$ be a ring, $R$ an A-algebra (commutative with identity), and let $B=R / I$, where $I$ is an ideal in $R$. Then there is a surjective B-algebra homomorphism $v: B \otimes_{R} P_{R / A}^{q} \rightarrow P_{B / A}^{q}$ such that $\operatorname{Ker}(v)$ is generated (as an ideal) by $\left\{1 \otimes d^{q} x \mid x \in I\right\}$, where $d^{q}=d_{R / A}^{q}: R \rightarrow$ $P_{R / A}^{q}$.

Lemma 4.3. Let $\left(A, \mathrm{~m}_{A}\right)$ be a Noetherian local ring, $R$ an A-algebra which is finitely generated as $A$-module, and $\left(B, \mathrm{~m}_{B}\right)=R_{\mathrm{m}}$, where $\mathrm{m}$ is a maximal ideal in $R$. Then $P_{\hat{B} / \hat{A}}^{q}=\hat{B} \otimes_{B} P_{B / A}^{q}$.

Proof. $B$ is a localization of $R$, so that $P_{B / A}^{q}=B \otimes_{R} P_{R / A}^{q}$. Therefore, it will suffice to show that $P_{\hat{B} / \hat{A}}^{q}=\hat{B} \otimes_{R} P_{R / A}^{q}$. Let $R$ have the $\mathrm{m}_{A}$-adic topology. Since $\hat{R}=\hat{A} \otimes_{A} R, P_{\hat{R} / \hat{A}}^{q}=\hat{R} \otimes_{R} P_{R / A}^{q}$; hence it is enough to prove that $P_{\hat{B} / \hat{A}}=\hat{B} \otimes_{\hat{R}} P_{\hat{R} / \hat{A}}^{q}$. Since $R$ is semilocal, $\hat{B}=\hat{R} / I$, where $I$ is generated by idempotent elements. By Lemma 4.2, it will suffice to show that $1 \otimes d^{q} x=$ 0 for every idempotent element $x \in I$, where $d^{q}=d_{\hat{R} / \hat{A}}^{q}$. For such an $x$, $\left(d^{q} x-x\right)^{q+1}=0$ [notation as in Lemma 3.7] and $x=x^{q+1}$, so that $d^{q} x=$ $d^{q} x^{q+1}=\sum_{i=1}^{q+1} n_{i} x^{i} d^{q} x^{q+1-i}$ for suitable $n_{i} \in \mathbf{Z}$. Therefore $1 \otimes d^{q} x=0$. Q.E.D.

Corollary 4.4. $\Delta_{q}\left(P_{\hat{B} / \hat{A}}^{q}\right)=\Delta_{q}\left(P_{B / A}^{q}\right) \cdot \hat{B}$. (The notation is as above.)

The corollary is an immediate consequence of Lemma 4.3 and the affine case of Lemma 2.7. In the case that $B$ is regular and $\Delta_{q}\left(P_{B / A}^{q}\right)$ is generated by a subset of a regular system of parameters, we conclude that $\Delta_{q}\left(P_{\hat{B} / \hat{A}}^{q}\right)$ is generated by a subset of a regular system of parameters. Thus, Theorem 4.1 is a direct consequence of the following lemma.

Lemma 4.5. Let $k$ be a field; let $\left(A, \mathrm{~m}_{A}\right)$ and $\left(B, \mathrm{~m}_{B}\right)$ be formal power series rings over $k$ in $m$ and $r$ variables respectively, where $m \geqslant r$. Let $g: A \rightarrow B$ be a local k-algebra homomorphism which makes $B$ a finite A-module, and assume that

(a) $\operatorname{dim}_{k} \mathrm{~m}_{B} /\left(g\left(\mathrm{~m}_{A}\right) B+\mathrm{m}_{B}^{2}\right)=1$,

(b) $\operatorname{dim}_{k} B / g\left(\mathrm{~m}_{A}\right) B=q+1$, 
(c) $\Delta_{q}\left(P_{B / A}^{q}\right)$ is generated by $q(m-r+1)$ elements of a regular system of parameters in $B$.

Then there exist minimal generating sets $\left\{s_{1}, \ldots, s_{m}\right\} \subset \mathrm{m}_{A}$ and $\left\{t_{1}, \ldots, t_{r}\right\} \subset \mathrm{m}_{B}$ such that $g: A \rightarrow B$ satisfies (i), (ii), and (iii) of Theorem 4.1.

The proof is given below, following some preliminary material.

If $M$ is a $B$-module, let $\bar{M}=M / \bigcap_{n=1}^{\infty} \mathrm{m}_{B}^{n} M$. We have $M=\bar{M}$ if and only if $M$ is separated in its $\mathrm{m}_{B}$-adic topology. In general, $\bar{M}$ is separated in its $\mathrm{m}_{B}$-adic topology; we call it the separated module associated to $M$. If $f: M \rightarrow N$ is a homomorphism of $B$-modules, then there is a homomorphism $\bar{f}: \bar{M} \rightarrow \bar{N}$.

LEmma 4.6. Consider an exact sequence of $B$-modules $M \rightarrow N \rightarrow P \rightarrow 0$, where $P$ is separated. Then the corresponding sequence of separated modules $\bar{M} \rightarrow \bar{N} \rightarrow P \rightarrow 0$ is also exact. (In particular, the hypotheses are satisfied if $\boldsymbol{P}$ is finitely generated.)

Let $\bar{d}^{q}=\nu \circ d_{B / k}^{q}: B \rightarrow \bar{P}_{B / k}^{q}$, where $\nu: P_{B / k}^{q} \rightarrow \bar{P}_{B / k}^{q}$ is the canonical map onto the associated separated module. Then every differential operator $D$ : $B \rightarrow B$ of order $\leqslant q$ is of the form $D=\phi^{\circ} \bar{d}^{q}$ for some $B$-module homomorphism $\phi: \bar{P}_{B / k}^{q} \rightarrow B$. (Note that $B$ is separated in the $\mathrm{m}_{B}$-adic topology.) $\bar{P}_{B / k}^{q}$ is a $B$-algebra because $\bigcap_{n=1}^{\infty} \mathrm{m}_{B}^{n} P_{B / k}^{q}$ is an ideal in $P_{B / k}^{q}$. Finally, $k \otimes_{B} \bar{P}_{B / k}^{q} \cong$ $B / \mathrm{m}_{B}^{q+1}$. (See Lemma 3.4.) Theorem 30.6 of [9] therefore implies that $\bar{P}_{B / k}^{q}$ is a finitely generated $B$-module.

LEMmA 4.7. Let $t_{1}, \ldots, t_{r}$ generate $\mathrm{m}_{B}$, so that $B=k\left[\left[t_{1}, \ldots, t_{r}\right]\right]$.

(i) If $i_{1}+\cdots+i_{r} \leqslant q$, then there is a unique differential operator $D=$ $D_{i_{1}} \cdots i_{r}: B \rightarrow B$ such that

$$
D\left(t_{1}^{d} \cdots t_{r}^{d_{r}}\right)=\left(\begin{array}{l}
d_{1} \\
i_{1}
\end{array}\right) \cdots\left(\begin{array}{l}
d_{r} \\
i_{r}
\end{array}\right) t_{1}^{d_{1}-i_{1}} \cdots t_{r}^{d_{r}-i_{r}}
$$

(ii) $\bar{P}_{B / k}^{q}$ is a free $B$-module, of rank $=\operatorname{dim}_{k} B / \mathrm{m}_{B}^{q+1}$.

Proof. We define a map $D: B \rightarrow B$ on monomials by the above formula and extend it linearly to arbitrary power series. One shows that if $b_{1}, \ldots, b_{q+1}$, $t$ are arbitrary elements of $B$, then

$$
\sum_{H}(-1)^{\operatorname{card}(H)}\left(\prod_{i \in H} b_{i}\right) D\left(\left(\prod_{i \notin H} b_{i}\right) t\right)=0 .
$$

( $H$ runs through all subsets of $\{1, \ldots, q+1\}$.) This identity implies that $D$ is a differential operator. Hence, we deduce a $B$-module homomorphism $\lambda: \bar{P}_{B / k}^{q} \rightarrow B$ such that $D=\lambda \circ \bar{d}^{q}$.

Let $\zeta_{i}=\bar{d}^{q} t_{i}-t_{i}, \quad i=1, \ldots, r$. Then $\bar{P}_{B / k}^{q}$ is generated as $B$-module by the monomials of degree $\leqslant q$ in $\zeta_{1}, \ldots, \zeta_{r}$. If $\lambda$ is the map constructed above, then 


$$
\begin{aligned}
& \lambda\left(\zeta_{1}^{i_{1}} \cdots \zeta_{r}^{i_{r}}\right)=1, \\
& \lambda\left(\zeta_{1}^{j_{1}} \cdots \zeta_{r}^{j_{r}}\right) \in \mathrm{m}_{B} \quad \text { if } j_{\nu} \neq i_{\nu} \text { for some } \nu .
\end{aligned}
$$

This shows that the monomials of degree $\leqslant q$ in $\zeta_{1}, \ldots, \zeta_{r}$ satisfy no $B$-linear relations. Q.E.D.

Proof of Lemma 4.5. We start with (minimal) generating sets $\left\{s_{1}, \ldots\right.$, $\left.s_{m}\right\} \subset \mathrm{m}_{A}$ and $\left\{t_{1}, \ldots, t_{r}\right\} \subset \mathrm{m}_{B}$, which will be modified as the proof proceeds. By (a), we may assume that $g\left(s_{i}\right)=t_{i}$ for $i=1, \ldots, r-1$ and that $t_{r} \notin g\left(\mathrm{~m}_{A}\right) B$. By (b), we may assume that the monomial $t_{r}^{q+1}$ occurs with nonzero coefficient in the power series expansion of $g\left(s_{r}\right)$ and does not occur in the power series expansions of $g\left(s_{r+1}\right), \ldots, g\left(s_{m}\right)$. Finally, we may assume that $g\left(s_{i}\right) \in \mathrm{m}_{B}^{2}$, $i=r, \ldots, m$. [If this is not the case, add $A$-linear combinations of $s_{1}, \ldots, s_{r-1}$ to $s_{i}$.] We then obtain

$$
g\left(s_{i}\right)=\sum_{j=1}^{\infty} b_{i j} t_{r}^{j}, \quad i=r, \ldots, m ;
$$

the $b_{i j}$ are elements of $k\left[\left[t_{1}, \ldots, t_{r-1}\right]\right]$ such that $b_{i j}$ is a nonunit if $j \leqslant q$, and $b_{i, q+1}$ is a unit if and only if $i=r$. We will assume that $b_{r, q+1}$ has constant term $=1$.

We now observe that there is a surjective $B$-algebra homomorphism $w: \bar{P}_{B / k}^{q}$ $\rightarrow P_{B / A}^{q}$ such that $\operatorname{Ker}(w)$ is generated by $\left\{\bar{d}^{q}(g(a)) \mid a \in A\right\}$. (See [4, Proposition 16.4.18] and Lemma 4.6.) Exactly as in the proof of Lemma 3.8 one shows that $\Delta_{q}\left(P_{B / A}^{q}\right)$ is generated by the elements $D^{(\lambda)}\left(g\left(s_{i}\right)\right)$, where $1 \leqslant \lambda \leqslant q, \quad r \leqslant i \leqslant m$, and $D^{(\lambda)}=D_{0} \ldots 0 \lambda: B \rightarrow B$ is defined as in Lemma 4.7. From (1) we obtain the congruences:

$$
\begin{array}{ll}
D^{(q)}\left(g\left(s_{r}\right)\right) \equiv(q+1) t_{r}+b_{r q}\left(\bmod \mathrm{m}_{B}^{2}\right), \\
D^{(\lambda)}\left(g\left(s_{i}\right)\right) \equiv b_{i \lambda} \quad\left(\bmod \mathrm{m}_{B}^{2}\right) \quad \text { if }(\lambda, i) \neq(q, r) .
\end{array}
$$

Now, (c) implies that $\left\{D^{(\lambda)}\left(g\left(s_{i}\right)\right) \mid 1 \leqslant \lambda \leqslant q\right.$ and $\left.r \leqslant i \leqslant m\right\}$ is a subset of a system of parameters in $B$. If $\operatorname{char}(k) \mid(q+1)$, then $(q+1) t_{r}=0$ and we conclude that $\left\{b_{i \lambda} \mid 1 \leqslant \lambda \leqslant q\right.$ and $\left.r \leqslant i \leqslant m\right\}$ is a subset of a system of parameters in $k\left[\left[t_{1}, \ldots, t_{r-1}\right]\right]$. If $\operatorname{char}(k) \nmid(q+1)$, we draw the "weaker" conclusion that $\left\{b_{i \lambda} \mid(i, \lambda) \neq(r, q)\right\}$ is a subset of a system of parameters.

Let $\left(S, \mathrm{~m}_{S}\right)=k\left[\left[s_{1}, \ldots, s_{r}\right]\right] \subset A$. Then $B / g\left(\mathrm{~m}_{S}\right) B$ has a basis consisting of the residues of $1, t_{r}, \ldots, t_{r}^{q}$. By Theorem 30.6 of [9], we conclude that $B$ is generated as $S$-module by $1, t_{r}, \ldots, t_{r}^{q}$. After a possible change of variables, we obtain

$$
g\left(s_{r}\right)=t_{r}^{q+1}+\sum_{j=1}^{q} g\left(a_{r j}\right) t_{r}^{j}
$$


Moreover $a_{r j} \in \mathrm{m}_{S}, j=1, \ldots, q$. We may also assume

$$
a_{r q}=0 \text { if } \operatorname{char}(k) \nmid(q+1) \text {. }
$$

[If this does not hold, replace $t_{r}$ with $t_{r}-(q+1)^{-1} g\left(a_{r q}\right)$ in the minimal generating set of $\mathrm{m}_{B}$.] Finally, we may assume

$$
g\left(s_{i}\right)=\sum_{j=1}^{q} g\left(a_{i j}\right) t_{r}^{j}, i=r+1, \ldots, m, \text { where all } a_{i j} \in \mathrm{m}_{S} .
$$

Let each of the nonunits $g\left(a_{i j}\right) \in B$ be expanded as power series in $t_{1}, \ldots$, $t_{r}$. In this way, (3) and (5) give power series expansions of $g\left(s_{r}\right), \ldots, g\left(s_{m}\right)$. If we compare these expansions with (1), then we obtain

$$
g\left(a_{i j}\right) \equiv b_{i j}\left(\bmod \mathrm{m}_{B}^{2}\right) \quad \text { when } r \leqslant i \leqslant m \text { and } 1 \leqslant j \leqslant q .
$$

Since $\left\{b_{i j}\right\}$ (possibly excluding $(i, j)=(r, q)$ ) is a subset of a system of parameters in $k\left[\left[t_{1}, \ldots, t_{r-1}\right]\right]$, it follows that we may change variables and assume that $\left\{a_{i j}\right\}$ (possibly excluding $(i, j)=(r, q)$ ) is a subset of $\left\{s_{1}, \ldots, s_{r-1}\right\}$. This implies that (i), (ii), and (iii) hold. Q.E.D.

REMARK 4.8. If $q(m-r+1)=r$ and $\operatorname{char}(k) \mid(q+1)$, then there are no points which satisfy the hypotheses of Theorem 4.1. This is because there are not enough parameters to serve as "coefficients" in conclusions (ii) and (iii). In the following example, we will demonstrate the nonexistence of smooth points more directly in the case $r=2, m=3, q=1$, and $\operatorname{char}(k)=2$.

EXAMPLE 4.9. Let $\operatorname{char}(k)=2$, and consider the $k$-algebra homomorphism $g: A \rightarrow B$, where $A=k\left[\left[s_{1}, s_{2}, s_{3}\right]\right], B=k\left[\left[t_{1}, t_{2}\right]\right]$, and

(a) $g\left(s_{1}\right)=t_{1}$,

(b) $g\left(s_{i}\right)=\Sigma_{j=1}^{\infty} b_{i j} t_{2}^{j}, i=2,3$, where $b_{i j} \in k\left[\left[t_{1}\right]\right]$.

Assume that $b_{21}$ and $b_{31}$ are nonunits but that some $b_{i j}$ is a unit, so that $2 \leqslant$ $\operatorname{dim}_{k} B / g\left(\mathrm{~m}_{A}\right) B<\infty$. Then $\Delta_{1}\left(P_{B / A}^{1}\right)=\Delta_{0}\left(\Omega_{B / A}^{1}\right)$ is generated by $\partial g\left(s_{2}\right) / \partial t_{2}$ and $\partial g\left(s_{3}\right) / \partial t_{2} ;$ moreover $\partial g\left(s_{i}\right) / \partial t_{2}=b_{i 1}+b_{i 3} t_{2}^{2}+\cdots$. Thus, $\Delta_{1}\left(P_{B / A}^{1}\right)$ cannot be generated by two elements of a regular system of parameters.

The following proposition gives an explicit description of the cotangent space of $S_{1}^{(q)}(f)$ in Theorem 4.1. It also shows that the converse of Lemma 4.5 is true.

Proposition 4.10. Let $A=k\left[\left[s_{1}, \ldots, s_{m}\right]\right], B=k\left[\left[t_{1}, \ldots, t_{r}\right]\right]$, and let $g: A \rightarrow B$ satisfy (i), (ii), and (iii) of Theorem 4.1. Then $\left(\Delta_{q}\left(P_{B / A}^{q}\right)+\mathrm{m}_{B}^{2}\right) / \mathrm{m}_{B}^{2}$ is the subspace of $\mathrm{m}_{B} / \mathrm{m}_{B}^{2}$ having the basis:

$$
\begin{aligned}
& \left\{\bar{t}_{i} \mid 1 \leqslant i \leqslant q(m-r+1)-1\right\} \cup\left\{\bar{t}_{r}\right\} \quad \text { if } \operatorname{char}(k) \nmid(q+1), \\
& \left\{\bar{t}_{i} \mid 1 \leqslant i \leqslant q(m-r+1)\right\} \quad \text { if } \operatorname{char}(k) \mid(q+1) .
\end{aligned}
$$

(The bars denote residue class modulo $\mathrm{m}_{B}^{2}$.) 
Proof. We use the notation of the proof of Lemma 4.5. Thus, $\Delta_{q}\left(P_{B / A}^{q}\right)$ is generated by $\left\{D^{(\lambda)} g\left(s_{i}\right) \mid 1 \leqslant \lambda \leqslant q\right.$ and $\left.r \leqslant i \leqslant m\right\}$. If $1 \leqslant \nu \leqslant r-1$, then $D^{(\lambda)} t_{\nu} t_{r}^{j}=\left({ }_{\lambda}^{j}\right) t_{\nu} t_{r}^{j-\lambda}$ (where $\left(_{\lambda}^{j}\right)=0$ if $\lambda>j$ ). Furthermore, $D^{(q)} t_{r}^{q+1}=(q+1) t_{r}$. Therefore, the set of initial forms of the elements $D^{(\lambda)} g\left(s_{i}\right)$ is

$$
\begin{aligned}
& \left\{t_{i} \mid 1 \leqslant i \leqslant q(m-r+1)-1\right\} \cup\left\{(q+1) t_{r}\right\} \quad \text { if } \operatorname{char}(k) \nmid(q+1), \\
& \left\{t_{i} \mid 1 \leqslant i \leqslant q(m-r+1)\right\} \quad \text { if } \operatorname{char}(k) \mid(q+1) .
\end{aligned}
$$

This implies that the desired conclusions hold. Q.E.D.

5. Some properties of fibre products. In this section we study some properties of fibre products of the singularity subschemes of a morphism of varieties. All of these are consequences of elementary properties of fibre products of schemes of finite type over the algebraically closed field $k$.

Proposition 5.1. Let $f_{j}: W_{j} \rightarrow Y, j=1, \ldots, d$, be morphisms over $k$, where $Y$ is a nonsingular variety and $W_{1}, \ldots, W_{d}$ are of finite type over $k$ and of pure dimension. If $W_{1} \times_{Y} \cdots \times_{Y} W_{d} \neq \varnothing$ and $Z$ is an irreducible component of $W_{1} \times_{Y} \cdots \times_{Y} W_{d}$, then

$$
\operatorname{dim}(Z) \geqslant \sum_{j=1}^{d} \operatorname{dim}\left(W_{j}\right)-(d-1) \cdot \operatorname{dim}(Y)
$$

Proof. Consider the morphism $f_{1} \times \cdots \times f_{d}: W_{1} \times \cdots \times W_{d} \rightarrow Y \times$ $\cdots \times Y$ of $d$-fold products over $\operatorname{Spec}(k)$, and let $\Delta \subset Y \times \cdots \times Y$ be the $d$-fold diagonal. Then

$$
W_{1} \times_{Y} \cdots \times_{Y} W_{d}=\left(f_{1} \times \cdots \times f_{d}\right)^{-1}(\Delta) \text {. }
$$

Since $Y$ is nonsingular, the sheaf of ideals defining $\Delta$ as a closed subscheme of $Y \times \cdots \times Y$ is generated locally by $(d-1) \cdot \operatorname{dim}(Y)$ elements at every point. Hence $\left(f_{1} \times \cdots \times f_{d}\right)^{-1}(\Delta)$ has the same property as a closed subscheme of $W_{1} \times \cdots \times W_{d}$. By Theorem 9.3 of [9], the codimension is $\leqslant(d-1) \cdot \operatorname{dim}(Y)$. Q.E.D.

Let $f: X \rightarrow Y$ be a morphism of nonsingular varieties. As in $\S 1$, we form fibre products $S_{1}^{\left(q_{1}\right)}(f) \times_{Y} \cdots \times_{Y} S_{1}^{\left(q_{d}\right)}(f)$ by using the restrictions of $f$, which send $S^{\left(q_{j}\right)}(f) \rightarrow Y, j=1, \ldots, d$. We recall that $\Sigma_{d}(f)$ is the complement in $X \times_{Y} \cdots \times_{Y} X$ of the union of all diagonals. The closed points of $\Sigma_{d}(f)$ will be called $d$-fold points of $f$. We also recall that

$$
\Sigma_{d}\left(f ; q_{1}, \ldots, q_{d}\right)=\Sigma_{d}(f) \cap\left(S_{1}^{\left(q_{1}\right)}(f) \times_{Y} \cdots \times_{Y} S_{1}^{\left(q_{d}\right)}(f)\right) .
$$

(In particular, $\Sigma_{d}\left(f ; q_{1}, \ldots, q_{d}\right)$ is an open subset of $S_{1}^{\left(q_{1}\right)}(f) \times_{Y} \cdots \times_{Y}$ $S_{1}^{\left(q_{d}\right)}(f)$.) 
CoROllaRY 5.2. If $Z$ is an irreducible component of $\Sigma_{d}\left(f ; q_{1}, \ldots, q_{d}\right)$ and $\Sigma_{d}\left(f ; q_{1}, \ldots, q_{d}\right) \neq \varnothing$, then $\operatorname{dim}(Z) \geqslant d r-(d-1) m-\Sigma_{j=1}^{d} q_{j}(m-r+1)$, where $r=\operatorname{dim}(X)$ and $m=\operatorname{dim}(Y)$. In particular, every irreducible component of $\Sigma_{d}(f)$ has dimension $\geqslant d r-(d-1) m$.

Proof. The conclusion follows from Proposition 5.1, because every irreducible component of $S_{1}^{\left(q_{j}\right)}(f)$ has dimension $\geqslant r-q_{j}(m+r+1)$. (See Corollary 3.9.) Q.E.D.

REMARK 5.3. The result of Corollary 5.2 is, of course, interesting only when $m \geqslant r$. We observe that this result could be considered the "easy half" of conclusion (i) of Theorem A.

Proposition 5.4. With the assumptions of Proposition 5.1, assume further that $W_{1}, \ldots, W_{d}$, and $Y$ are smooth over $k$. Let $x_{1}, \ldots, x_{d}$ be closed points of $W_{1}, \ldots, W_{d}$ respectively, and assume that $f_{1}\left(x_{1}\right)=\cdots=f_{d}\left(x_{d}\right)=y \in Y$. Let $\left(O_{y}, \mathrm{~m}_{y}\right)=O_{Y, y},\left(O_{x_{j}}, \mathrm{~m}_{x_{j}}\right)=O_{W_{j}, x_{j}}$, and let $\rho_{j}: \mathrm{m}_{y} / \mathrm{m}_{y}^{2} \rightarrow \mathrm{m}_{x_{j}} / \mathrm{m}_{x_{j}}^{2}$ be induced by $f_{j}, j=1, \ldots, d$. Then $W_{1} \times_{Y} \cdots \times_{Y} W_{d}$ is smooth and of the smallest possible dimension at $\left(x_{1}, \ldots, x_{d}\right)$ if and only if $\operatorname{dim}_{k}\left(N_{1}+\cdots+N_{d}\right)$ $=\operatorname{dim}_{k}\left(N_{1}\right)+\cdots+\operatorname{dim}_{k}\left(N_{d}\right)$, where $N_{j}=\operatorname{Ker}\left(\rho_{j}\right) \subset \mathrm{m}_{y} / \mathrm{m}_{y}^{2}, j=1, \ldots, d$.

Proof. Let $\xi=\left(x_{1}, \ldots, x_{d}\right) \in W_{1} \times \cdots \times w_{d}$ and $\eta=(y, \ldots, y) \in$ $Y \times \cdots \times Y$. (These $d$-fold products are taken over $\operatorname{Spec}(k)$.) Then $\mathrm{m}_{\eta} / \mathrm{m}_{\eta}^{2} \cong$ $\Sigma_{j=1}^{d} \bigoplus \mathrm{m}_{y} / \mathrm{m}_{y}^{2}$, and if $J \subset O_{\eta}$ defines the $d$-fold diagonal $\Delta$, then

$$
\left(J+\mathrm{m}_{\eta}^{2}\right) / \mathrm{m}_{\eta}^{2} \cong\left\{\left(s_{1}, \ldots, s_{d}\right) \in \sum_{j=1}^{d} \bigoplus \mathrm{m}_{y} / \mathrm{m}_{y}^{2} \mid s_{1}+\cdots+s_{d}=0\right\} .
$$

On the other hand, $f_{1} \times \cdots \times f_{d}$ induces the linear map $\rho: \mathrm{m}_{\eta} / \mathrm{m}_{\eta}^{2} \rightarrow \mathrm{m}_{\xi} / \mathrm{m}_{\xi}^{2}=$ $\Sigma_{j=1}^{d} \bigoplus \mathrm{m}_{x_{j}} / \mathrm{m}_{x_{j}}^{2}$, where $\rho\left(s_{1}, \ldots, s_{d}\right)=\left(\rho_{1}\left(s_{1}\right), \ldots, \rho_{d}\left(s_{d}\right)\right)$.

$\left(f_{1} \times \cdots \times f_{d}\right)^{-1}(\Delta)$ is smooth at $\left(x_{1}, \ldots, x_{d}\right)$ if and only if

$$
\operatorname{Ker}(\rho) \cap\left[\left(J+\mathrm{m}_{\eta}^{2}\right) / \mathrm{m}_{\eta}^{2}\right]=(0) .
$$

This holds if and only if $\operatorname{dim}_{k}\left(N_{1}+\cdots+N_{d}\right)=\operatorname{dim}_{k}\left(N_{1}\right)+\cdots+$ $\operatorname{dim}_{k}\left(N_{d}\right)$. Q.E.D.

COROLlARY 5.5. Let $f: X^{r} \rightarrow Y^{m}$ be a morphism of nonsingular varieties, and let $x_{1}, \ldots, x_{d}$ be distinct closed points of $X$ such that $f\left(x_{1}\right)=\cdots=f\left(x_{d}\right)$ $=y \in Y$. Then $\Sigma_{d}(f)$ is smooth and of dimension $d r-(d-1) m$ at $\left(x_{1}, \ldots, x_{d}\right)$ if and only if

$$
\operatorname{dim}_{k}\left(\bigcap_{j=1}^{d} \operatorname{Im}\left((d f)_{x_{j}}\right)\right)=\sum_{j=1}^{d} \operatorname{dim}_{k}\left(\operatorname{Im}\left((d f)_{x_{j}}\right)\right)-(d-1) m,
$$

where $(d f)_{x_{j}}: T(X)_{x_{j}} \rightarrow T(Y)_{y}$ is the map of Zariski tangent spaces induced by $f$. 
COROLlARY 5.6. With the assumptions and notation of Corollary 5.5, assume also that $x_{j} \in S^{\left(q_{j}\right)}(f), j=1, \ldots, d$. Let $I_{j} \subset O_{x_{j}}$ be the ideal $\Delta_{q_{j}}\left(P_{X / Y}^{q_{j}}\right)_{x_{i}}$. Let $f$ induce $\lambda: \mathrm{m}_{y} / \mathrm{m}_{y}^{2} \rightarrow \mathrm{m}_{x_{1}} / \mathrm{m}_{x_{1}}^{2}$ and $\rho_{j}: \mathrm{m}_{y} / \mathrm{m}_{y}^{2} \rightarrow$ $\mathbf{m}_{x_{j}} /\left(\mathbf{m}_{x_{j}}^{2}+I_{j}\right), j=1, \ldots, d$. Let $N_{j}=\operatorname{Ker}\left(\rho_{j}\right), j=1, \ldots, d$. If $\Sigma_{d}\left(f ; 0, q_{2}, \ldots, q_{d}\right)$ is smooth and of the smallest possible dimension at $\left(x_{1}, \ldots, x_{d}\right)$, then $\Sigma_{d}\left(f ; q_{1}, \ldots, q_{d}\right)$ is smooth and of the smallest possible dimension at $\left(x_{1}, \ldots, x_{d}\right)$ if and only if there exist elements $t_{1}, \ldots$, $t_{q_{1}(m-r+1)}$ of $I_{1}$ such that $\left\{t_{i} \mid 1 \leqslant i \leqslant q_{1}(m-r+1)\right\} \subset \mathrm{m}_{x_{1}} / \mathrm{m}_{x_{1}}^{2}$ is linearly independent modulo the subspace $\lambda\left(N_{2}+\cdots+N_{d}\right) \subset \mathrm{m}_{x_{1}} / \mathrm{m}_{x_{1}}^{2}$.

Proof. Each of the statements which must be shown to be equivalent implies that $S_{1}^{\left(q_{1}\right)}(f)$ is smooth at $x_{1}$. Assume that this is true. Since $\Sigma_{d}\left(f ; 0, q_{2}, \ldots, q_{d}\right)$ is smooth at $\left(x_{1}, \ldots, x_{d}\right)$, Proposition 5.4 implies that $\operatorname{Ker}(\lambda) \cap$ $\left(N_{2}+\cdots+N_{d}\right)=(0)$. Therefore, we must prove that $\Sigma_{d}\left(f ; q_{1}, \ldots, q_{d}\right)$ is smooth and of the smallest possible dimension at $\left(x_{1}, \ldots, x_{d}\right)$ if and only if $\operatorname{Ker}(\nu) \cap \lambda\left(N_{2}+\cdots+N_{d}\right)=(0)$, where $\nu: \mathrm{m}_{x_{1}} / \mathrm{m}_{x_{1}}^{2} \rightarrow \mathrm{m}_{x_{1}} /\left(\mathrm{m}_{x_{1}}^{2}+I_{1}\right)$ is the canonical surjection. The equivalence of these two statements follows from Proposition 5.4 because $\rho_{1}=\nu \circ \lambda$.

6. A lemma about projective embeddings. In this section we prove the existence of projective embeddings having certain properties.

Definition 6.1. Let $V$ be a closed subvariety of $\mathrm{P}^{N}=\operatorname{Proj} k\left[T_{0}, \ldots, T_{N}\right]$, and let $H \subset \mathrm{P}^{N}$ be a hyperplane. Let $\mathrm{P}^{N}-H$ be identified with $\operatorname{Spec} k\left[T_{1}, \ldots, T_{n}\right]$ (after a linear change of variables). A linear coordinate function on $U=V-V \cap$ $H$ is an element of $\Gamma\left(U, O_{V}\right)$ induced by a linear combination of the elements 1 , $T_{1}, \ldots, T_{n}$.

Consider an affine variety $U\left(\right.$ over $k$ ) and points $x_{1}, \ldots, x_{d} \in U$. Let $b_{1}, \ldots, b_{d}$ be positive integers. For $j=1, \ldots, d$ we have ring homomorphisms $\phi_{j}: \Gamma\left(U, O_{U}\right) \rightarrow O_{x_{j}} / \mathrm{m}_{x_{j}}^{b_{j}}$, where $\left(O_{x_{j}}, \mathrm{~m}_{x_{j}}\right)$ is the local ring $O_{U, x_{j}}$. Therefore, we have a ring homomorphism $\phi: \Gamma\left(U, O_{U}\right) \rightarrow A$, where $A$ is the Artinian ring $\Pi_{j=1}^{d} O_{x_{j}} / \mathrm{m}_{x_{j}}^{b_{j}}$. It is well known that $\phi$ is surjective.

Definition 6.2. Let $U$ and $\phi: \Gamma\left(U, O_{U}\right) \rightarrow A$ be as above. Let $E$ be a subset of $\Gamma\left(U, O_{U}\right)$. We say that $A$ is spanned by $E$ if $A$ is spanned (as $k$-vector space) by $\phi(E)$.

LEMMA 6.3. Let $b_{1}, \ldots, b_{d}$ be positive integers and let $V$ be a projective variety over $k$. There exists a projective embedding $V \subset \mathrm{P}^{N}$ (for some $N$ ) such that if $x_{1}, \ldots, x_{d}$ are closed points of $V$ and $H \subset \mathrm{p}^{N}$ is a hyperplane such that $x_{j} \in U=V-V \cap H$ for $j=1, \ldots, d$, then $\Pi_{j=1}^{d} O_{x_{j}} / \mathrm{m}_{x_{j}}^{b_{j}}$ is spanned by the linear coordinate functions on $U$, where $\left(0_{x_{j}}, \mathrm{~m}_{x_{j}}\right)=0_{V, x_{j}}, j=1, \ldots, d$.

Proof. Let an embedding $V \subset \mathrm{P}^{n}$ be given. We reduce to the case $V=$ 
$\mathrm{P}^{n}$ by considering the surjections $\mathrm{O}_{\mathrm{P} n, x_{j}} \rightarrow \mathrm{O}_{V, x_{j}}$. For each $g>0$, let $\sigma_{g}: \mathrm{P}^{n}$ $\hookrightarrow \mathrm{P}^{N}$ be the $g$-tuple embedding (defined by global sections of the sheaf $O(g)$ on $\left.\mathbf{P}^{n}\right)$. Lemma 6.3 is a consequence of the following result.

LEMMA 6.4. If $g \geqslant\left(\sum_{j=1}^{d} b_{j}\right)-1$, then the conclusion of Lemma 6.3 holds for the embedding $\sigma_{g}: \mathrm{P}^{n} \hookrightarrow \mathrm{P}^{N}$.

Proof. Given a hyperplane $H \subset \mathrm{P}^{N}$ such that $\sigma_{g}\left(x_{j}\right) \notin H$ for $j=1, \ldots, d$, we change $H$ so that $\sigma_{g}^{-1}(H)$ is a hyperplane in $\mathrm{P}^{n}$ counted $g$ times. Such a change multiplies the linear coordinate functions by an element which is a unit in each $O_{x_{j}}$

We now choose a basis $\left\{T_{0}, \ldots, T_{n}\right\}$ for $\Gamma\left(P^{n}, O(1)\right)$ so that $\sigma_{g}^{-1}(H)$ is the hypersurface $T_{0}^{g}=0$; we identify $\mathrm{P}^{n}-\sigma_{g}^{-1}(H)$ with $\operatorname{Spec}(B)$, where $B=$ $k\left[T_{1}, \ldots, T_{n}\right]$. Let $m_{j} \subset B$ be the maximal ideal corresponding to $x_{j}$, so that $\Pi_{j=1}^{d} O_{x_{j}} / \mathrm{m}_{x_{j}}^{b_{j}} \cong \Pi_{j=1}^{d} B / \mathrm{m}_{j}^{b_{j}}$.

We choose an $(n-2)$-subspace $\Lambda$ of the hyperplane $T_{0}=0$ which does not meet any of the lines $\overline{x_{i} x_{j}}$, and we change the coordinates so that this subspace has the equations $T_{0}=T_{1}=0$. Thus, if $x_{j}$ has homogeneous coordinates $\left(1, c_{j 1}\right.$, $\ldots, c_{j n}$ ), it follows that $c_{11}, \ldots, c_{d 1}$ are distinct. (Check this by projecting from $\Lambda$.) For each $i$, we set $h_{j}\left(T_{1}\right)=\prod_{i \neq j}\left(T_{1}-c_{i 1}\right)^{b_{i}}$. Then $h_{j} \notin \mathrm{m}_{j}$, but $h_{j} \in$ $\mathrm{m}_{i}^{b_{i}}$ if $i \neq j$. Multiplying $h_{j}$ by the monomials of degree $\leqslant b_{j}-1$ in $T_{1}-c_{j 1}$, $\ldots, T_{n}-c_{j n}$, we obtain polynomials of degree $\leqslant g$ lying in $m_{i}^{b_{i}}$ for $i \neq j$ whose residue classes span $B / \mathrm{m}_{j}^{b_{j}}$. This completes the proof. Q.E.D.

Corollary 6.5. Let $x_{1}, \ldots, x_{d}$ be distinct closed points of $\mathrm{P}^{n}$. Assume that $g \geqslant 2 d-1$. Then the tangent spaces $t_{\sigma_{g}(\mathrm{P} n), \sigma_{g}\left(x_{j}\right)}, j=1, \ldots, d$, span a linear subspace of $\mathrm{P}^{N}$ of the largest possible dimension, namely $d(n+1)-1$.

REMARK 6.6. This corollary is a stronger version of Corollary 1 of [11, $\S 2]$.

7. The $d$-fold points of a generic projection. Let $V^{r}$ be a nonsingular quasiprojective variety over $k$. When a projective embedding $V^{r} \subset \mathrm{P}^{n}$ and a closed point $x \in V$ are given, $t_{V, x}$ will denote the r-subspace of $\mathrm{P}^{n}$ tangent to $V$ at $x$. If $L \subset \mathrm{P}^{n}$ is an $(n-m-1)$-subspace with $L \cap \bar{V}=\varnothing$ [so that $m \geqslant r$ ], we will denote by $\pi_{L}: \mathrm{P}^{n}-L \rightarrow \mathrm{P}^{m}$ the projection from $L$ and by $\pi: V \rightarrow \mathrm{P}^{m}$ the restriction of $\pi_{L}$ to $V$. We will denote by $\pi_{L}\left(t_{V, x}\right)$ the linear subspace $\pi_{L}\left(t_{V, x}-L\right)$ $\subset \mathbf{P}^{\boldsymbol{m}}$.

Proposition 7.1. Let $V^{r}$ be a nonsingular subvariety of $\mathrm{P}^{n}, L \subset \mathrm{P}^{n}$ an $(n-m-1)$-subspace with $L \cap \bar{V}=\varnothing$. Let $x \in V$ be a closed point, set $y=$ $\pi(x) \in \mathbf{P}^{m}$, and let $\rho: \mathrm{m}_{y} / \mathrm{m}_{y}^{2} \rightarrow \mathrm{m}_{x} / \mathrm{m}_{x}^{2}$ be induced by $\pi^{*}: \mathrm{O}_{\mathrm{p} m, y} \rightarrow \mathrm{O}_{V, x}$. The following statements are equivalent (where $i$ is any positive integer): 
(a) $\operatorname{dim}_{k}(\operatorname{Ker}(\rho)) \geqslant m-r+i$.

(b) $x \in S_{i}(\pi)$.

(c) $\operatorname{dim}\left(L \cap t_{V, x}\right) \geqslant i-1$.

(d) $\operatorname{dim}\left(\pi_{L}\left(t_{V, x}\right)\right) \leqslant r-i$.

Proof. (a) $\Leftrightarrow$ (b) is a consequence of Proposition 2.4. To prove (c) $\Leftrightarrow(d)$, observe that the closure (in $\mathrm{P}^{n}$ ) of $\pi_{L}^{-1}\left(\pi_{L}(x)\right.$ ) is the subspace spanned by $L$ and $x$, so that the closure of $\pi_{L}^{-1}\left(\pi_{L}(x)\right) \cap t_{V, x}$ is the subspace spanned by $x$ and $L \cap t_{V, x}$. Since $\pi_{L}^{-1}\left(\pi_{L}(x)\right) \cap t_{V, x}$ is a typical fibre of $\pi_{L} \mid\left(t_{V, x}-L\right)$, the equivalence of (c) and (d) is now easily established.

In proving (c) $\Leftrightarrow(a)$, we assume that equality holds in (c), and we select homogeneous coordinates $T_{0}, \ldots, T_{n}$ on $\mathrm{P}^{n}$ such that:

(i) $L$ is the zero locus of $\left\{T_{0}, \ldots, T_{m}\right\}$,

(ii) $x=(1,0, \ldots, 0)$,

(iii) $t_{V, x}$ is the zero locus of $\left\{T_{r+1-i}, \ldots, T_{n-i}\right\}$.

We set $t_{0}=1$ and work with affine coordinates $t_{1}, \ldots, t_{n}$ in an affine open neighborhood of $x \in V$. By (i), (ii), and (iii), it follows that $\operatorname{coker}(\rho) \cong$ $\mathrm{m}_{x} /\left(\mathrm{m}_{x}^{2}+\pi^{*}\left(\mathrm{~m}_{y}\right) \cdot O_{x}\right)$ is spanned by the residues of $t_{n-i+1}, \ldots, t_{n}$ and that these elements are linearly independent. Hence, equality holds in (a). Q.E.D.

Proposition 7.2. Let $V \subset \mathrm{P}^{n}, L \subset \mathrm{P}^{n}$, and $\pi: V \rightarrow \mathrm{P}^{m}$ be as before; let $x_{1}, \ldots, x_{d}$ be distinct closed points of $V$ such that $\pi\left(x_{1}\right)=\cdots=\pi\left(x_{d}\right)=$ $y \in \mathrm{P}^{m}$ so that $\left(x_{1}, \ldots, x_{d}\right) \in \Sigma_{d}(\pi)$. Then $\Sigma_{d}(\pi)$ is smooth (over $k$ ) at $\left(x_{1}, \ldots, x_{d}\right)$ and locally of dimension $d r-(d-1) m$ if and only if

$$
\operatorname{dim}\left(\bigcap_{j=1}^{d} \pi_{L}\left(t_{V, x_{j}}\right)\right)=\sum_{j=1}^{d} \operatorname{dim}\left(\pi_{L}\left(t_{V, x_{j}}\right)\right)-(d-1) m .
$$

PROOF. It is well known that there is a bijective map

$\alpha:$ Vector subspaces of $\left.T\left(\mathbf{P}^{m}\right)_{y}\right\} \rightarrow$ \{Subspaces of $\mathbf{P}^{m}$ containing $y$ \} which preserves dimension and intersections. By choosing coordinates as in the preceding proof, one shows that $\alpha$ sends $(d \pi)_{x_{j}}\left(T(V)_{x_{j}}\right)$ to $\pi_{L}\left(t_{V, x_{j}}\right)$. Therefore, the desired conclusion follows from Corollary 5.5. Q.E.D.

We set $d_{*}=[m /(m-r)]+1$ in the case $m>r$ and $d_{*}=r+1$ in the case $m=r$. Consider the following property of a projective embedding $V \subset \mathbf{P}^{n}$.

( $\alpha)$ If $d \leqslant d_{*}$, and $x_{1}, \ldots, x_{d}$ are distinct closed points of $V$, then the tangent spaces $t_{V, x_{1}}, \ldots, t_{V, x_{d}}$ span a subspace of $\mathrm{P}^{n}$ of the largest possible dimension, viz. $d(r+1)-1$.

LEMMA 7.3. Every nonsingular quasi-projective variety has a projective embedding which satisfies $(\alpha)$. 
The proof uses the techniques of $\S 6$ or of $[11, \S 2]$.

In the statement of the following proposition, we identify $\Sigma_{d}(\pi)$ with a subscheme of $V \times \cdots \times V(d$-fold product over $\operatorname{Spec}(k))$.

Proposition 7.4. Let $V^{r}$ be a nonsingular subvariety of $\mathrm{P}^{n}$, and assume that $(\alpha)$ holds. If $\pi: V \rightarrow \mathrm{P}^{m}$ is induced by projection from a generic $(n-m-1)$ subspace $L \subset \mathrm{P}^{n}$, then for all sequences $i_{1}, \ldots, i_{d}$ of nonnegative integers, $\Sigma_{d}(\pi)$ $\cap\left(S_{i_{1}}(\pi) \times \cdots \times S_{i_{d}}(\pi)\right)$ is either empty or of pure dimension $d r-(d-1) m-$ $\Sigma_{j=1}^{d} i_{j}\left(m-r+i_{j}\right)$. (We set $S_{0}=V$.) In particular, if this integer is negative, then $\Sigma_{d} \cap\left(S_{i_{1}} \times \cdots \times S_{i_{d}}\right)=\varnothing$.

COROLlARY 7.5. If $\pi: V^{r} \rightarrow \mathrm{P}^{m}$ is a generic projection, then for every $i \geqslant 0, S_{i}(\pi)$ is either empty or of pure codimension $i(m-r+i)$ in $V$.

Proof of Proposition 7.4. I claim that it will suffice to show that the conclusion holds for the (finitely many) $d$-tuples $\left(i_{1}, \ldots, i_{d}\right)$ such that $d \leqslant d_{*}$ and $i_{j} \leqslant r$ for $j=1, \ldots, d$. The restriction $i_{j} \leqslant r$ involves no loss of generality because $S_{i}(f)=\varnothing$ when $i>r$ for any morphism $f: V \rightarrow \mathrm{P}^{m}$. If $m>r$, then this special case of the conclusion (more precisely the case $d=d_{*}$ and $i_{1}=\cdots=i_{d}$ $=0)$ implies that $\operatorname{card}\left(\pi^{-1}(\pi(x))\right) \leqslant d_{*}-1$ for all $x \in V$. This will imply that $\Sigma_{d}(\pi)=\varnothing$ whenever $d \geqslant d_{*}$. If $m=r$, it is only necessary to prove the result when $i_{j}>0$ for $j=1, \ldots, d$. [This is because $\pi$ may be assumed to be finite. Thus, if $d \leqslant d^{\prime}$ and $\Sigma_{d^{\prime}}(\pi) \rightarrow \Sigma_{d}(\pi)$ sends $\left(x_{1}, \ldots, x_{d^{\prime}}\right) \rightarrow\left(x_{1}, \ldots, x_{d}\right)$, the fibres are finite. If $i_{d+1}=\cdots=i_{d^{\prime}}=0$, then the result for $\left(i_{1}, \ldots, i_{d}\right.$, $0, \ldots, 0)$ follows from the result for $\left(i_{1}, \ldots, i_{d}\right)$.] Therefore, if $m=r$ and we have proved the result for the case $d \leqslant d_{*}=r+1$, we conclude that $\Sigma_{r+1}(\pi)$ $\cap\left(S_{1}(\pi) \times \cdots \times S_{1}(\pi)\right)=\varnothing$. This implies that the result holds for all $d$-tuples $\left(i_{1}, \ldots, i_{d}\right)$.

Therefore, we may fix $d \leqslant d_{*}$ and $\left(i_{1}, \ldots, i_{d}\right)$ and then show that there is a dense open subset of the Grassmann variety $G(n, n-m-1)$ whose points correspond to linear subspaces $L \subset \mathrm{P}^{n}$ such that $\pi=\pi_{L} \mid V$ satisfies the conclusions of the proposition relative to this particular $d$-tuple. (Observe that the intersection of finitely many dense open subsets is still a dense open subset.) We recall that $\operatorname{dim} G(n, n-m-1)=(m+1)(n-m)$. Let $G_{0} \subset G$ consist of all $(n-m-1)$ subspaces $L \subset \mathrm{P}^{n}$ such that $L \cap \bar{V}=\varnothing$.

Let $U_{d} \subset V \times \cdots \times V(d$ copies $)$ consist of all $d$-tuples of distinct points, and let $W \subset U_{d} \times G_{0}$ consist of all $\left(x_{1}, \ldots, x_{d}, L\right)$ such that

(i) $\operatorname{dim}\left(L \cap M\left(x_{1}, \ldots, x_{d}\right)\right) \geqslant d-2$, where $M\left(x_{1}, \ldots, x_{d}\right)$ is the $(d-1)$ subspace of $\mathbf{P}^{n}$ spanned by $x_{1}, \ldots, x_{d}$;

(ii) $\operatorname{dim}\left(L \cap t_{V, x_{j}}\right) \geqslant i_{j}-1$ for $j=1, \ldots, d$.

As in [11], we see that (i) is equivalent to saying that $\pi\left(x_{1}\right)=\cdots=\pi\left(x_{d}\right)$, or equivalently that $\left(x_{1}, \ldots, x_{d}\right) \in \Sigma_{d}(\pi)$. Proposition 7.1 implies that (ii) is 
equivalent to saying that $x \in S_{i j}(\pi)$ for $j=1, \ldots, d$. By looking at suitable incidence correspondences, one shows that $W$ is closed in $U \times G_{0}$.

Let $p: W \rightarrow U_{d}$ send $\left(x_{1}, \ldots, x_{d}, L\right) \rightarrow\left(x_{1}, \ldots, x_{d}\right)$. By Lemma 7.9 below (applied in the case that $q=n-m-1$ and $r_{1}=\cdots=r_{d}=r$ ):

$$
\operatorname{dim}\left(p^{-1}\left(x_{1}, \ldots, x_{d}\right)\right)=(m+1)(n-m)-\sum_{j=1}^{d} i_{j}\left(m-r+i_{j}\right)-(d-1) m,
$$

so that $\operatorname{dim}(W)=\operatorname{dim}(G(n, n-m-1))+d r-(d-1) m-\Sigma_{j=1}^{d} i_{j}\left(m-r+i_{j}\right)$. Thus, if $L$ is chosen to lie in a dense open subset of $G_{0}$, the dimension of the fibre of $W$ above $L$ will be $\operatorname{dim}(W)-\operatorname{dim}\left(G_{0}\right)$, or else the fibre will be empty. In other words,

$$
\operatorname{dim}\left(\Sigma_{d} \cap\left(S_{i_{1}} \times \cdots \times S_{i_{d}}\right)\right)=d r-(d-1) m-\sum_{j=1}^{d} i_{j}\left(m-r+i_{j}\right) \text {. Q.E.D. }
$$

THEOREM 7.6. Let $V^{r} \subset \mathrm{P}^{n}$ be as in Proposition 7.4. If $\pi: V^{r} \rightarrow \mathrm{P}^{m}$ is a generic projection (where $r \leqslant m \leqslant 2 r$ ), then for every $d \geqslant 0, \Sigma_{d}(\pi)$ is either empty or smooth (over $k$ ) and of pure dimension $d r-(d-1) m$.

REMARK 7.7. Statement (iii) of Theorem 1 of [11] is equivalent to smoothness of $\Sigma_{d}(\pi)$ at points $\left(x_{1}, \ldots, x_{d}\right)$ such that $x_{j} \notin S_{1}(\pi)$ for $j=1, \ldots, d$. The proof of Theorem 7.6 is similar to the proof of Lemma 7 of [11]; the essential difference is that Corollary 7.10 must now be used to calculate the dimension of the fibres of $F_{\sigma} \rightarrow G_{\sigma}$.

REMARK 7.8. When $m=r$ it is necessary to prove smoothness only at points $\left(x_{1}, \ldots, x_{d}\right)$ such that $x_{j} \in S_{1}(\pi)$ for all $j$. In fact, if $x_{d} \notin S_{1}(\pi)$, a straightforward application of Proposition 7.2 shows that $\left(x_{1}, \ldots, x_{d}\right)$ is a smooth point of $\Sigma_{d}(\pi)$ if and only if $\left(x_{1}, \ldots, x_{d-1}\right)$ is a smooth point of $\Sigma_{d-1}(\pi)$. (The essential observation is that $\pi_{L}\left(t_{V, x_{d}}\right)=\mathrm{P}^{r}$ because $x_{d} \notin S_{1}(\pi)$.)

Proof of TheOREM 7.6. We must show that if $\pi: V \rightarrow \mathrm{P}^{m}$ is a generic projection, then for all $d$-tuples $\left(i_{1}, \ldots, i_{d}\right)$ of nonnegative integers, there do not exist points $\left(x_{1}, \ldots, x_{d}\right)$ such that (i) $\left(x_{1}, \ldots, x_{d}\right)$ is a nonsmooth point of $\Sigma_{d}(\pi)$ and (ii) $x_{j} \in S_{i_{j}}(\pi)-S_{i_{j}+1}(\pi)$. As in the proof of Proposition 7.4, we observe that we may fix $\left(i_{1}, \ldots, i_{d}\right)$ [with $d \leqslant d_{*}$ ] and prove that there is a dense open subset of $G(n, n-m-1)$ whose points correspond to $(n-m-1)$ subspaces $L \subset \mathrm{P}^{n}$ such that $\pi=\pi_{L} \mid V$ has the desired properties relative to the given $d$-tuple $\left(i_{1}, \ldots, i_{d}\right)$.

We define $G_{0} \subset G(n, n-m-1)$ and $U_{d} \subset V \times \cdots \times V$ as before. We consider a point $\left(z_{1}, \ldots, z_{d}\right) \in U_{d}$ and $L \in G_{0}$ such that $\pi\left(z_{1}\right)=\cdots=\pi\left(z_{d}\right)$ and $z_{j} \in S_{i_{j}}(\pi)-S_{i_{j}+1}(\pi)$ for $j=1, \ldots, d$. By Propositions 7.1 and $7.2, \Sigma_{d}(\pi)$ fails to be smooth at $\left(z_{1}, \ldots, z_{d}\right)$ if and only if there exists a linear subspace $\Lambda^{\prime} \subset \bigcap_{j=1}^{d} \pi_{L}\left(t_{V, z_{j}}\right)$ such that 


$$
\operatorname{dim}\left(\Lambda^{\prime}\right)=m-d(m-r)-\sum_{j=1}^{d} i_{j}+1 .
$$

Setting $\Lambda=\pi_{L}^{-1}\left(\Lambda^{\prime}\right)$, we have

$$
\begin{aligned}
& \operatorname{dim}(\Lambda)=\operatorname{dim}\left(\Lambda^{\prime}\right)+n-m=n-d(m-r)-\sum_{j=1}^{d} i_{j}+1, \\
& \operatorname{dim}\left(\Lambda \cap t_{V, z_{j}}\right) \geqslant \operatorname{dim}\left(\Lambda^{\prime}\right)+i_{j} \quad \text { for } j=1, \ldots, d .
\end{aligned}
$$

For any integer $\sigma \geqslant 0$, let $E_{\sigma} \subset U_{d} \times G(n, q)$, where $q=n-m-1$, be the subset consisting of all closed points $\left(x_{1}, \ldots, x_{d}, L\right)$ such that $L \cap \bar{V}=\varnothing$ and

(a) $\operatorname{dim}(L \cap M) \geqslant d-2$, where $M$ is spanned by $x_{1}, \ldots, x_{d}$;

(b) $\operatorname{dim}\left(L \cap t_{V, x_{j}}\right) \geqslant i_{j}-1$ for $j=1, \ldots, d$;

(c) there is a subspace $\Lambda \subset \mathrm{P}^{n}$ with $\operatorname{dim}(\Lambda)=q+\sigma+1, L \subset \Lambda, x_{j} \in \Lambda$, and $\operatorname{dim}\left(t_{V, x_{j}} \cap \Lambda\right) \geqslant \sigma+i_{j}$ for $j=1, \ldots, d$.

Since we want to show that $\Sigma_{d}(\pi)$ is smooth if $L$ is chosen to lie in some dense open subset of $G(n, q)$, the proof will be finished if we can show that $E_{\sigma}$ is a constructible subset of $U_{d} \times G(n, q)$ with $\operatorname{dim}\left(E_{\sigma}\right)<\operatorname{dim}(G(n, q))$ in the case that $\sigma=m-d(m-r)-\Sigma_{j=1}^{d} i_{j}+1$.

We will estimate $\operatorname{dim}\left(E_{\sigma}\right)$ by studying $F_{\sigma}$, which is defined to be the subset of $U_{d} \times G(n, \nu) \times G(n, q)$, where $\nu=q+\sigma+1$, consisting of all $\left(x_{1}, \ldots, x_{d}\right.$, $\Lambda, L)$ such that $L \cap \bar{V}=\varnothing,($ a) and (b) above are satisfied, and

(c') $L \subset \Lambda, x_{j} \in \Lambda$ and $\operatorname{dim}\left(\Lambda \cap t_{V, x_{j}}\right) \geqslant \sigma+i_{j}$ for $j=1, \ldots, d$.

By Lemma 8 of [11], $F_{\sigma}$ is locally closed in $U_{d} \times G(n, v) \times G(n, q)$. Let $p_{13}: U_{d} \times G(n, \nu) \times G(n, q) \rightarrow U_{d} \times G(n, q)$ be the projection; then $E_{\sigma}=$ $p_{13}\left(F_{\sigma}\right)$. We conclude that $E_{\sigma}$ is constructible, and $\operatorname{dim}\left(E_{\sigma}\right) \leqslant \operatorname{dim}\left(F_{\sigma}\right)$.

Let $G_{\sigma} \subset U_{d} \times G(n, v)$ be the closed subset consisting of all $\left(x_{1}, \ldots, x_{d}, \Lambda\right)$ with $x_{j} \in \Lambda$ and $\operatorname{dim}\left(\Lambda \cap t_{V, x_{j}}\right) \geqslant \sigma+i_{j}$ for $j=1, \ldots, d$. Using Lemma 7.11 below, we see that the dimension of $G_{\sigma}$ is

$$
\begin{aligned}
d r+(\nu+1)(n-\nu) & -d \sigma((n-\nu)-(r-\sigma))-d(n-\nu) \\
& -((n-\nu)-(r-2 \sigma)) \sum_{j=1}^{d} i_{j}-\sum_{j=1}^{d} i_{j}^{2} .
\end{aligned}
$$

Recalling that $\nu=n-m+\sigma$, so that $n-\nu=m-\sigma$, we find

$$
\begin{aligned}
\operatorname{dim}\left(G_{\sigma}\right)= & d r+(n-m+\sigma+1)(m-\sigma)-d \sigma(m-r)-d(m-\sigma) \\
& -(m-r+\sigma) \sum_{j=1}^{d} i_{j}-\sum_{j=1}^{d} i_{j}^{2} .
\end{aligned}
$$

The projection $p_{12}: U_{d} \times G(n, \nu) \times G(n, q) \rightarrow U_{d} \times G(n, \nu)$ induces a surjection $\phi: F_{\sigma} \rightarrow G_{\sigma}$. If $\left(x_{1}, \ldots, x_{d}, \Lambda\right)$ is a point of $G_{\sigma}$, then the fibre over this point 
is isomorphic to the locally closed subvariety of $G(\nu, q)$ which parameterizes all $L \subset \Lambda$ which satisfy conditions (a) and (b) and also $L \cap \bar{V}=\varnothing$. By Corollary 7.10 below [applied with $\nu$ replacing $n$ ], the dimension of any fibre of $\phi$ is $(q+1)(\nu-q)-(d-1)(\nu-q-1)$. Recalling that $\nu=q+\sigma+1$ and $q=n-$ $m-1$, we obtain

$$
\begin{aligned}
\operatorname{dim}\left(F_{\sigma}\right)= & \operatorname{dim}\left(G_{\sigma}\right)+(n-m)(\sigma+1)-(d-1) \sigma \\
= & (m+1)(n-m)+m-d(m-r)-(m-r) \sum_{j=1}^{d} i_{j} \\
& -\sum_{j=1}^{d} i_{j}^{2}+\sigma\left(m-d(m-r)-\sum_{j=1}^{d} i_{j}\right)-\sigma^{2} .
\end{aligned}
$$

In the case $\sigma=m-d(m-r)-\Sigma_{j=1}^{d} i_{j}+1$, this becomes

$$
\operatorname{dim}\left(F_{\sigma}\right)=\operatorname{dim}(G(n, n-m-1))-(m-r) \sum_{j=1}^{d} i_{j}+\sum_{j=1}^{d}\left(i_{j}-i_{j}^{2}\right)-1 .
$$

Therefore, $\operatorname{dim}\left(F_{\sigma}\right) \leqslant \operatorname{dim}(G(n, n-m-1))-1$; in particular, $\operatorname{dim}\left(E_{\sigma}\right)<$ $\operatorname{dim}(G(n, n-m-1))$. Q.E.D.

LEMMA 7.9. Let $L_{1}, \ldots, L_{d}$ be subspaces of $\mathbf{P}^{n}$, and let $x_{j} \in L_{j}$ for $j=$ $1, \ldots, d$. Let $r_{j}=\operatorname{dim}\left(L_{j}\right)$, and assume that $L_{1}, \ldots, L_{d}$ span a subspace of $\mathrm{p}^{n}$ of the largest possible dimension, viz. $\Sigma_{j=1}^{d}\left(r_{j}+1\right)-1$. Let $M \subset \mathrm{p}^{n}$ be the $(d-1)$-subspace spanned by $x_{1}, \ldots, x_{d}$, let $i_{1}, \ldots, i_{d}$ be nonnegative integers with $i_{j} \leqslant r_{j}$, and let $q$ satisfy $n>q \geqslant \Sigma_{j=1}^{d} i_{j}+d-2$. Finally, let $Z \subset G(n, q)$ be the locally closed subset consisting of all q-subspaces $L \subset \mathrm{P}^{n}$ such that

(0) $x_{j} \notin L$ for $j=1, \ldots, d$,

(1) $\operatorname{dim}\left(L \cap L_{j}\right) \geqslant i_{j}-1$ for $j=1, \ldots, d$,

(2) $\operatorname{dim}(L \cap M) \geqslant d-2$.

Then, $\operatorname{dim}(Z) \leqslant(q+1)(n-q)-(d-1)(n-q-1)-\Sigma_{j=1}^{d} i_{j}\left(n-q-1-r_{j}+i_{j}\right)$, and equality holds if and only if $q \leqslant n-r_{j}+i_{j}-1$ for all $j$.

We note that condition (1) is vacuous for those values of $j$ with $i_{j}=0$.

Corollary 7.10. Let the notation be as in Lemma 7.9. Assume that there is an integer $\sigma \geqslant 0$ such that $n=q+\sigma+1$ and $r_{j}=\sigma+i_{j}$ for $j=1$, $\ldots, d$. Then $\operatorname{dim}(Z)=(q+1)(n-q)-(d-1)(n-q-1)$.

Proof of Lemma 7.9. Let $G_{0} \subset G(d-1, d-2)$ parameterize $(d-2)$ subspaces $M^{\prime} \subset M$ with $x_{j} \notin M^{\prime}$ for $j=1, \ldots, d$. When $1 \leqslant j \leqslant d$, let $G_{j} \subset$ $G\left(r_{j}, i_{j}-1\right)$ be the dense open subset which parameterizes $\left(i_{j}-1\right)$-subspaces $M_{j} \subset L_{j}$ with $x_{j} \notin M_{j}$. (If $i_{j}=0$, we take $G_{j}=\operatorname{Spec}(k)$ ). Let $Y=G_{0} \times \cdots \times$ $G_{d}$, and let $T \subset G(n, q) \times Y$ consist of all $\left(L, M_{0}, \ldots, M_{d}\right)$ with $M_{j} \subset L$ for $j=0, \ldots, d$. Let $p_{1}$ and $p_{2}$ be the projections of $G(n, q) \times Y$ to the first and 
second factors, and let $q_{2}: T \rightarrow Y$ be the restriction of $p_{2}$. Then $Z=p_{1}(T)$, so that $\operatorname{dim}(Z) \leqslant \operatorname{dim}(T)$; equality holds if and only if there exists a $q$-subspace $L \subset \mathbb{P}^{n}$ such that equality holds in (1) and (2). On the other hand, let $y=$ $\left(M_{0}, \ldots, M_{d}\right) \in Y$. Then $\left(L, M_{0}, \ldots, M_{d}\right) \in T$ if and only if $M_{*} \subset L$, where $M_{*}$ is spanned by $M_{0}, \ldots, M_{d}$. Hence, $q_{2}^{-1}(y)$ is irreducible and of the same dimension as $G(n-e-1, q-e-1)$, where $e=\operatorname{dim}\left(M_{*}\right)=\Sigma_{j=1}^{d} i_{j}+d-2$. Therefore

$$
\begin{aligned}
\operatorname{dim}(T) & =\operatorname{dim}(Y)+\operatorname{dim}(G(n-e-1, q-e-1)) \\
& =d-1+\sum_{j=1}^{d} i_{j}\left(r-i_{j}+1\right)+(q-e)(n-q) \\
& =(q+1)(n-q)-(d-1)(n-q-1)-\sum_{j=1}^{d} i_{j}\left(n-q-1-r+i_{j}\right) . \quad \text { Q.E.D. }
\end{aligned}
$$

Lemma 7.11. Let $L_{1}, \ldots, L_{d}$ be $r$-subspaces of $\mathrm{P}^{n}$, and let $x_{j} \in L_{j}$ be a closed point, for $j=1, \ldots, d$. Assume that $\operatorname{dim}(L)=(d+1)(r+1)-1$, where $L$ is spanned by $L_{1}, \ldots, L_{d}$. Let $\nu, \sigma, i_{1}, \ldots, i_{d}$ be nonnegative integers such that $\sigma+i_{j} \leqslant r$ for $j=1, \ldots, d$, and

$$
d(\sigma+1)+\sum_{j=1}^{d} i_{j}-1 \leqslant \nu \leqslant n-1 .
$$

Let $H \subset G(n, v)$ consist of all $\nu$-subspaces $\Lambda \subset \mathrm{P}^{n}$ such that $x_{j} \in \Lambda$ and $\operatorname{dim}\left(\Lambda \cap L_{j}\right) \geqslant \sigma+i_{j}$ for $j=1, \ldots, d$. Then $H$ is irreducible, and

$$
\begin{aligned}
\operatorname{dim}(H) \leqslant & (\nu+1)(n-\nu)-d \sigma((n-\nu)-(r-\sigma))-d(n-\nu) \\
& -((n-\nu)-(r-2 \sigma)) \sum_{j=1}^{d} i_{j}-\sum_{j=1}^{d} i_{j}^{2} .
\end{aligned}
$$

Equality holds if and only if $\nu \leqslant n-r+\sigma+i_{j}$ for all $j$.

The proof of this lemma is similar to the proof of Lemma 9 of [11]. We omit the details.

8. The morphism $\Phi$. Let $V^{+}$be a nonsingular closed subvariety of $\mathrm{P}^{n}=$ Proj $k\left[X_{0}, \ldots, X_{n}\right]$ and let $m$ be an integer such that $n>m \geqslant \operatorname{dim}(V)$. We construct a morphism which parameterizes projections of $V$ to $\mathrm{P}^{m}$ (see Definition 8.1) and study its behavior under certain coordinate changes.

Let $N=(m+1)(n+1)-1$ and $\mathbf{P}^{N}=\operatorname{Proj} k\left[\tau_{i j} \mid 0 \leqslant i \leqslant m ; 0 \leqslant j \leqslant n\right]$. The homogeneous coordinates of a closed point can be regarded as entries of an $(m+1) \times(n+1)$ matrix. Let $U \subset \mathrm{P}^{N}$ consist of all points where this matrix has rank $(m+1)$. If $z=\left(\beta_{i j}\right) \in U$, let $L_{z} \subset \mathrm{P}^{n}$ be the $(n-m-1)$-subspace 
given by the vanishing of the linear forms $\Sigma_{j=0}^{n} \beta_{i j} T_{j}, i=0, \ldots, m$. Since $m \geqslant$ $\operatorname{dim}(V)$, there is a dense open subset $Z \subset U$ such that $L_{z} \cap V=\varnothing$ for all $z \in Z$.

Definition 8.1. Let $z=\left(\beta_{i j}\right) \in Z \subset \mathrm{P}^{N}$. We define $\Phi_{z}: V \rightarrow \mathrm{P}^{m}$ to be the unique morphism such that if $x=\left(a_{0}, \ldots, a_{n}\right) \in V \subset \mathrm{P}^{n}$, then $\Phi_{z}(x)=$ $\left(c_{0}, \ldots, c_{m}\right) \in \mathrm{P}^{m}=\operatorname{Proj} k\left[Y_{0}, \ldots, Y_{m}\right]$, where $c_{i}=\Sigma_{j=0}^{n} \beta_{i j} a_{j}$. [Equivalently, we can specify that $\Phi_{z}^{*}: \Gamma\left(\mathbf{P}^{m}, O(1)\right) \rightarrow \Gamma\left(\mathbf{P}^{n}, O(1)\right)$ satisfies $\Phi_{z}^{*}\left(Y_{i}\right)=\Sigma_{j=0}^{n} \beta_{i j} X_{j}$, $i=0, \ldots, m$.] Finally, we define $\Phi: V \times Z \rightarrow \mathrm{P}^{m} \times Z$ to be the unique morphism which satisfies $\Phi(x, z)=\left(\Phi_{z}(x), z\right)$. (The product sign " $x$ " without a subscript will henceforth denote the product $x_{k}$ (taken over $\left.\operatorname{Spec}(k)\right)$.)

LEMMA 8.2. With the above notation, $\Phi_{z}: V \rightarrow \mathrm{P}^{m}$ is induced by projection from $L_{z}$. If $z$ and $z^{\prime}$ are points of $Z$ such that $L_{z}=L_{z^{\prime}}$, then $\Phi_{z}$ and $\Phi_{z^{\prime}}$ differ by an automorphism of $\mathbf{P}^{m}$.

LEMMA 8.3. Let $\left\{S_{0}, \ldots, S_{m}\right\}$ and $\left\{T_{0}, \ldots, T_{n}\right\}$ be bases of $\Gamma\left(\mathrm{P}^{m}, O(1)\right)$ and $\Gamma\left(\mathrm{P}^{n}, O(1)\right)$ respectively. Let $z_{0} \in Z$. Assume that $L_{z_{0}} \subset \mathrm{P}^{n}$ is given by the vanishing of $T_{0}, \ldots, T_{m}$ and that $\Phi_{z_{0}}^{*}\left(S_{i}\right)=T_{i}, i=0, \ldots, m$. Then there is a basis $\left\{\sigma_{i j} \mid 0 \leqslant i \leqslant m\right.$ and $\left.0 \leqslant j \leqslant n\right\}$ of $\Gamma\left(\mathbf{P}^{N}, O(1)\right)$ such that (i) if $z \in Z \subset \mathrm{P}^{N}$ has homogeneous coordinates $\left(b_{i j}\right)$ relative to $\left\{\sigma_{i j}\right\}$, then $\Phi_{z}^{*}\left(S_{i}\right)=\Sigma_{j=0}^{n} b_{i j} T_{j}$, for $i=0, \ldots, m$;

(ii) $z_{0}$ has homogeneous coordinates $\left(\delta_{i j}\right)$ relative to $\left\{\sigma_{i j}\right\}$, where $\delta_{i j}=1$ (resp. 0) if $i=j$ (resp. $i \neq j)$.

PROOF. $X_{\nu}=\Sigma_{j=0}^{n} \alpha_{\nu j} T_{j}, \nu=0, \ldots, n$, and $S_{i}=\Sigma_{\lambda=0}^{m} \gamma_{i \lambda} Y_{\lambda}, i=0$, $\ldots, m$, for suitable $\alpha_{\nu j}$ and $\gamma_{i \lambda} \in k$. We set

$$
\sigma_{i j}=\sum_{\lambda=0}^{m} \sum_{\nu=0}^{n} \gamma_{i \lambda} \alpha_{\nu j} \tau_{\lambda \nu} .
$$

The matrix of coefficients which expresses $\left\{\sigma_{i j}\right\}$ in terms of $\left\{\tau_{\lambda \nu}\right\}$ is invertible because it is the Kronecker product of two invertible matrices. If $z \in Z$ has homogeneous coordinates $\left(b_{i j}\right)$ relative to $\left\{\sigma_{i j}\right\}$, then

$$
b_{i j}=\sum_{\lambda=0}^{m} \sum_{\nu=0}^{n} \gamma_{i \lambda} \alpha_{\nu j} \beta_{\lambda \nu},
$$

where $\left\{\beta_{\lambda \nu}\right\}$ is a set of homogeneous coordinates of $z$ relative to $\left\{\tau_{\lambda \nu}\right\}$. Therefore

$$
\begin{aligned}
\Phi_{z}^{*}\left(S_{i}\right) & =\sum_{\lambda=0}^{m} \gamma_{i \lambda} \Phi_{z}^{*}\left(Y_{\lambda}\right) \\
& =\sum_{\lambda=0}^{m} \sum_{\nu=0}^{n} \sum_{j=0}^{n} \gamma_{i \lambda} \beta_{\lambda \nu} \alpha_{\nu j} T_{j}=\sum_{j=0}^{n} b_{i j} T_{j} .
\end{aligned}
$$

This proves (i), and (ii) is clear. Q.E.D. 
9. The singularity subschemes of $\Phi$. We again fix a nonsingular projective variety $V$ and an integer $m \geqslant r=\operatorname{dim}(V)$. Let $d_{*}=[m /(m-r)]+1$ (respectively $d_{*}=r+1$ ) if $m \geqslant r+1$ (respectively $m=r$ ). We set $q_{*}=[r /(m-r+1)]+1$ in either case. (The brackets denote integer part.) We also assume that the embedding $V \subset \mathrm{P}^{n}$ satisfies:

The conclusion of Lemma 6.3 holds with

$$
d=d_{*} \text { and } b_{1}=\cdots=b_{d}=q_{*}+1 \text {. }
$$

(See also Lemma 6.4.) We set $N=(m+1)(n+1)-1$ and consider the dense open subset $Z \subset \mathrm{P}^{N}$ and the morphism $\Phi: V \times Z \longrightarrow \mathrm{P}^{m} \times Z$ as in Definition 8.1.

Theorem 9.1. Let $V \subset \mathrm{P}^{n}$ be as above. Assume that $d \leqslant d_{*}$ and $0 \leqslant q_{j}$ $\leqslant q_{*}, j=1, \ldots, d$. Then $\Sigma_{d}\left(\Phi ; q_{1}, \ldots, q_{d}\right)$ is smooth over $k$ and has pure dimension $d r-(d-1) m-\Sigma_{j=1}^{d} q_{j}(m-r+1)+\operatorname{dim}(Z)$. In particular, $S_{1}^{(q)}(\Phi)$ has pure codimension $q(m-r+1)$ in $V \times Z$ whenever $0 \leqslant q \leqslant q_{*}$.

Proof. Corollary 5.2 implies that every irreducible component of $\Sigma_{d}\left(\Phi ; q_{1}, \ldots, q_{d}\right)$ has dimension $\geqslant d r-(d-1) m-\Sigma_{j=1}^{d} q_{j}(m-r+1)+$ $\operatorname{dim}(Z)$. In proving the opposite inequality we will regard $\Sigma_{d}\left(\Phi ; q_{1}, \ldots, q_{d}\right)$ as a subscheme of $V \times \cdots \times V \times Z$. Therefore,

$$
\left(x_{1}, \ldots, x_{d}, z\right) \in \Sigma_{d}\left(\Phi ; q_{1}, \ldots, q_{d}\right)
$$

if and only if (a) $\Phi_{z}\left(x_{1}\right)=\cdots=\Phi_{z}\left(x_{d}\right)$ and (b) $\left(x_{j}, z\right) \in S_{1}^{\left(q_{j}\right)}(\Phi), j=1$, $\ldots, d$. If $\operatorname{Spec}(k) \rightarrow Z$ corresponds to the closed point $z \in Z$, then $S_{1}^{\left(q_{j}\right)}\left(\Phi_{z}\right) \cong$ $\operatorname{Spec}(k) \times{ }_{Z} S_{1}^{\left(q_{j}\right)}(\Phi)$. Thus (b) holds if and only if $x_{j} \in S_{1}^{\left(q_{j}\right)}\left(\Phi_{z}\right), j=1, \ldots, d$.

Let $\left(x_{1}, \ldots, x_{d}, z_{0}\right)$ be a closed point of $W=\Sigma_{d}\left(\Phi ; q_{1}, \ldots, q_{d}\right)$. We will show that there is a subspace $\Lambda \subset \mathrm{P}^{N}$ such that

(i) $z_{0} \in \Lambda$;

(ii) $\operatorname{dim}(\Lambda)=(d-1) m+\Sigma_{j=1}^{d} q_{j}(m-r+1)$;

(iii) if $S=\left(x_{1}, \ldots, x_{d}\right) \times \Lambda$, then $S \cap W$ is the point $\left(x_{1}, \ldots, x_{d}, z_{0}\right)$ with reduced structure.

In other words, $S \cap W$ is nonsingular and of pure codimension $(d-1) m+$ $\Sigma_{j=1}^{d} q_{j}(m-r+1)$ in $S$. Since $\left(x_{1}, \ldots, x_{d}, z_{0}\right)$ is an arbitrary closed point of $W$, we will obtain the desired conclusion.

In constructing $\Lambda$, we fix homogeneous coordinate systems $\left\{T_{0}, \ldots, T_{n}\right\}$ on $\mathrm{P}^{n}$ and $\left\{S_{0}, \ldots, S_{m}\right\}$ on $\mathrm{P}^{m}$ which satisfy (1) through (6) below.

(1) $x_{1}=(1,0, \ldots, 0)$, and $x_{j}=(1,0, \ldots, 0,1,0, \ldots, 0), j=2, \ldots$, $d$. (The 0 th and $(n-j+2)$ th coordinates of $x_{j}$ are nonzero.)

(2) $L_{z_{0}} \subset \mathrm{P}^{n}$ is the subspace $T_{0}=\cdots=T_{m}=0$, and $\Phi_{z_{0}}^{*}: \Gamma\left(\mathrm{P}^{m}, O(1)\right)$ $\rightarrow \Gamma\left(\mathbb{P}^{n}, O(1)\right)$ satisfies $\Phi_{z_{0}}^{*}\left(S_{i}\right)=T_{i}, i=0, \ldots, m$. (In particular, $\Phi_{z_{0}}\left(x_{1}\right)=$ $\cdots=\Phi_{z_{0}}\left(x_{d}\right)=y=(1,0, \ldots, 0) \in \mathrm{P}^{m}$.) 
(3) For $j=1, \ldots, d$, the $(n-r)$-subspace $T_{0}=\cdots=T_{r-1}=0$ meets the tangent space $t_{V, x_{j}} \subset \mathrm{P}^{n}$ in exactly one point.

This is possible by Proposition 7.1. (Note that $\left(x_{j}, z_{0}\right) \notin S_{2}(\Phi)$, so that $x_{j} \notin S_{2}\left(\Phi_{z_{0}}\right)$.)

We now work with affine coordinate functions $t_{1}, \ldots, t_{n}$ on $V$ (we set $\left.t_{0}=1\right)$. By $(*)$ we can modify $t_{n-d+2}, \ldots, t_{n}$ to obtain the following stronger version of (1), where $\left(O_{x_{j}}, m_{x_{j}}\right)=O_{V, x_{j}}$.

(4) If $2 \leqslant \nu \leqslant d$ and $1 \leqslant j \leqslant d$, then

$$
\begin{aligned}
& t_{n-\nu+2} \equiv 1\left(\bmod \mathrm{m}_{x_{j}}^{q_{*}+1}\right) \text { if } \nu=j, \\
& t_{n-\nu+2} \equiv 0\left(\bmod \mathrm{m}_{x_{j}}^{q_{*}+1}\right) \text { if } \nu \neq j .
\end{aligned}
$$

By (3), the elements $t_{i}=\Phi_{z_{0}}^{*}\left(s_{i}\right), i=1, \ldots, r-1$, are linearly independent modulo $\mathrm{m}_{x_{j}}^{2}$ for all $j$, where $s_{1}, \ldots, s_{m}$ are affine coordinates on $\mathbf{P}^{m}$ in a neighborhood of $y$. By $(*)$ we may also assume

(5) If $q_{j}>0$, then $t_{1}, \ldots, t_{r-1}, t_{m+j}$ generate $\mathrm{m}_{x_{j}}$, while $t_{m+j^{\prime}} \in \mathrm{m}_{x_{j}}^{q *+2}$ if $q_{j^{\prime}}>0$ and $j^{\prime} \neq j$.

(6) If $q_{j}>0$ and $1 \leqslant l \leqslant q_{*}$, then $t_{\nu(j, l)} \equiv t_{m+j}^{l}\left(\bmod \mathrm{m}_{x_{j}}^{q_{*}^{+1}}\right)$, where $v(j, l)=m+(l-1) d+j$, while if $q_{j^{\prime}}>0$ and $j^{\prime} \neq j$, then $t_{\nu\left(j^{\prime}, l\right)} \equiv 0$ $\left(\bmod \mathrm{m}_{x_{j}}^{q * 1}\right)$.

Having good coordinate systems on $\mathbf{P}^{m}$ and $\mathbf{P}^{n}$, we now fix a homogeneous coordinate system $\left\{\sigma_{i \nu} \mid 0 \leqslant i \leqslant m\right.$ and $\left.0 \leqslant \nu \leqslant n\right\}$ on $\mathrm{P}^{N}$ which satisfies the conclusions of Lemma 8.3. We take $\Lambda \subset \mathrm{P}^{N}$ to be the linear subspace given by the vanishing of all $\sigma_{i \nu}-\delta_{i \nu} \sigma_{00}$ except those which satisfy either

(7a) $n-d+2 \leqslant \nu \leqslant n$ and $1 \leqslant i \leqslant m$, or

(7b) $r \leqslant i \leqslant m$ and $\nu=\nu(j, l)$ for some $j, l$ such that $q_{j}>0$ and $1 \leqslant l \leqslant q_{j}$.

I claim that (i), (ii) and (iii) hold for this $\Lambda$. Of course, (i) and (ii) are clear. To establish (iii), we get $Z^{\prime}=Z \cap \Lambda$ and consider the morphism $\Phi^{\prime}=$ $\left.\Phi\right|_{V \times Z^{\prime}}: V \times Z^{\prime} \rightarrow \mathrm{P}^{m} \times Z^{\prime}$. Thus if $\Phi$ is regarded as a morphism of schemes over $Z$, then $\Phi^{\prime}$ is obtained by the base extension $Z^{\prime} \rightarrow Z$. Therefore $S_{1}^{(q)}\left(\Phi^{\prime}\right)=$ $S_{1}^{(q)}(\Phi) \times{ }_{Z} Z^{\prime}$ for all $q \geqslant 0$, by Proposition 3.3. Using elementary properties of fibre products one shows $\Sigma_{d}\left(\Phi^{\prime}\right)=\Sigma_{d}(\Phi) \times{ }_{Z} Z^{\prime}$. In other words $S_{1}^{(q)}\left(\Phi^{\prime}\right)=$ $S_{1}^{(q)}(\Phi) \cap\left(V \times Z^{\prime}\right)$ in $V \times Z$, and $\Sigma_{d}\left(\Phi^{\prime}\right)=\Sigma_{d}(\Phi) \cap\left(V \times \cdots \times V \times Z^{\prime}\right)$ in $V \times \cdots \times V \times Z$. Moreover,

$$
\Sigma_{d}\left(\Phi ; q_{1}, \ldots, q_{d}\right)=\Sigma_{d}(\Phi) \cap p_{1}^{-1}\left(S_{1}^{\left(q_{1}\right)}(\Phi)\right) \cap \cdots \cap p_{d}^{-1}\left(S_{1}^{\left(q_{d}\right)}(\Phi)\right)
$$

where $p_{j}: V \times \cdots \times V \times Z \rightarrow V \times Z$ sends $\left(x_{1}, \ldots, x_{d}, z\right) \rightarrow\left(x_{j}, z\right)$. Therefore, (iii) is a consequence of the following two lemmas.

LEMMA 9.2. If $q_{j}>0$, then $S_{1}^{\left(q_{j}\right)}\left(\Phi^{\prime}\right) \cap\left(\left\{x_{j}\right\} \times Z^{\prime}\right)=\left\{x_{j}\right\} \times\left(\Lambda_{j} \cap Z^{\prime}\right)$, where 
$\Lambda_{j}$ is the subspace of $\Lambda$ given by the vanishing of $\left\{\sigma_{i \nu} \mid r \leqslant i \leqslant m\right.$ and $\nu=\nu(j, l)$ for some $\left.l \in\left\{1, \ldots, q_{j}\right\}\right\}$.

LEMMA 9.3. $\Sigma_{d}\left(\Phi^{\prime}\right) \cap\left(\left(x_{1}, \ldots, x_{d}\right) \times Z^{\prime}\right)=\left(x_{1}, \ldots, x_{d}\right) \times\left(\Lambda_{0} \cap Z^{\prime}\right)$, where $\Lambda_{0} \subset \Lambda$ is the subspace of $\Lambda$ given by the vanishing of $\left\{\sigma_{i \nu} \mid 1 \leqslant i \leqslant m\right.$ and $n-$ $d+2 \leqslant \nu \leqslant n\}$.

Proof of Lemma 9.2. Consider the statement about $S_{1}^{\left(q_{1}\right)}\left(\Phi^{\prime}\right) \cap\left(\left\{x_{1}\right\} \times Z^{\prime}\right)$. To simplify notation we set $x=x_{1}$ and $q=q_{1}$. Let $\left(A, \mathrm{~m}_{A}\right)$ and $\left(B, \mathrm{~m}_{B}\right)$ be the local rings on $\mathrm{P}^{m} \times Z^{\prime}$ at $\left(y, z_{0}\right)$ and on $V \times Z^{\prime}$ at $\left(x, z_{0}\right)$ respectively, and let $\Phi^{\prime *}: A \rightarrow$ $B$ be induced by $\Phi^{\prime}$. In particular, $A$ and $B$ are localizations of $O_{y} \otimes_{k} R$ and $O_{x} \otimes_{k}$ $R$, where $\left(O_{x}, \mathrm{~m}_{x}\right)=\mathcal{O}_{V, x},\left(\mathcal{O}_{y}, \mathrm{~m}_{y}\right)=\mathcal{O}_{\mathrm{p} m, y}$, and $\left(R, \mathrm{~m}_{R}\right)=\mathcal{O}_{Z^{\prime} z_{0}}$. Moreover, $\Phi^{\prime *}$ is an $R$-algebra homomorphism. As minimal generating set of $\mathrm{m}_{R}$ we take $\left\{\sigma_{i \nu}\right\}$ where $(i, v)$ runs through all pairs such that either $(7 \mathrm{a})$ or $(7 \mathrm{~b})$ holds. Then

(8a) $\Phi^{\prime} *\left(\sigma_{i \nu}\right)=\sigma_{i \nu}$ for all $(i, \nu)$;

(8b) $\Phi^{\prime *}\left(s_{i}\right)=u_{i}$ for $i=1, \ldots, r-1$;

(8c) $\Phi^{\prime *}\left(s_{i}\right)=u_{i}+\Sigma_{(j, l)} \sigma_{i, \nu(j, l)} t_{\nu(j, l)}$ for $i=r, \ldots, m$, where $u_{i}=t_{i}+$ $\sum_{\nu=n-d+2}^{n} \sigma_{i v} t_{\nu}, i=1, \ldots, m$. $(j, l)$ runs through all pairs such that $q_{j}>0$ and $1 \leqslant$ $l \leqslant q_{j}$. (We have written $\sigma_{i v}, s_{i}$ and $t_{\nu}$ to denote the elements $1 \otimes \sigma_{i \nu}, s_{i} \otimes 1$ and $t_{\nu} \otimes 1$, respectively.)

Since $\left\{u_{1}, \ldots, u_{r-1}, t_{m+1}\right\}$ is a minimal generating set of $m_{x} B$, Proposition 3.10 implies that there are $R$-linear differential operators $D^{(\mu)}: B \rightarrow B, \mu=1, \ldots, q$, such that

(9) if $b \equiv\left(\Pi_{\nu=1}^{r-1} u_{\nu}^{i}\right) t_{m+1}^{l}\left(\bmod \mathrm{m}_{x}^{q+1} B\right)$, then

$$
D^{(\mu)} b \equiv\left(\begin{array}{l}
l \\
\mu
\end{array}\right)\left(\prod_{\nu=1}^{r-1} u_{\nu}^{i}\right) t_{m+1}^{l-\mu}\left(\bmod \mathrm{m}_{x}^{q+1-\mu} B\right) ;
$$

(10) $\Delta_{q}\left(P_{B / A}^{q}\right)$ is generated by the elements $D^{(\mu)} \Phi^{\prime *}\left(s_{i}\right)$, where $1 \leqslant \mu \leqslant q$ and $r \leqslant i \leqslant m$.

By (5), (6), and (9), we see that if $1 \leqslant l \leqslant q$, then

$$
\begin{aligned}
D^{(\mu)} t_{\nu(1, l)} & \equiv 1\left(\bmod \mathrm{m}_{x} B\right) \quad \text { if } \mu=l, \\
& \equiv 0\left(\bmod \mathrm{m}_{x} B\right) \text { if } \mu \neq l .
\end{aligned}
$$

Using this, together with (8c) and the $R$-linearity of $D^{(\mu)}$, and observing that (4) implies $D^{(\mu)} u_{i} \in \mathrm{m}_{x} B, i=r, \ldots, m$, we conclude that $D^{(\mu)} \Phi^{\prime *}\left(s_{i}\right) \equiv \sigma_{i, \nu(1, \mu)}\left(\bmod \mathrm{m}_{x} B\right)$ whenever $1 \leqslant \mu \leqslant q$ and $r \leqslant i \leqslant m$. By (10), this implies that $\mathrm{m}_{x} B+\Delta_{q}\left(P_{B / A}^{q}\right)$ $=\mathrm{m}_{x} B+J$, where $J \subset B$ is generated by $\left\{\sigma_{i, \nu(1, l)} \mid r \leqslant i \leqslant m\right.$ and $\left.1 \leqslant l \leqslant q\right\}$. Since $\Delta_{q}\left(P_{B / A}^{q}\right) \subset B$ corresponds to the subscheme $S_{1}^{(q)}\left(\Phi^{\prime}\right)$, the proof of the case $j=1$ is complete.

The proof is entirely similar in the case $2 \leqslant j \leqslant d$. The idea is that equations like (8b) and (8c) hold with $s_{i}$ and $u_{i}$ replaced by $s_{i}-\sigma_{i, n-j+2}$ and $u_{i}-\sigma_{i, n-j+2}$ 
respectively. With this change, Proposition 3.10 can be applied as in the case considered above. Q.E.D.

The proof of Lemma 9.3 is straightforward and will be omitted. Let us simply observe that if $z=\left(b_{i v}\right)$ is a point of $Z^{\prime}$, then $\Phi_{z}\left(x_{1}\right)=\cdots=\Phi_{z}\left(x_{d}\right)$ if and only if $b_{i \nu}=0$ for all $i \in\{1, \ldots, m\}$ and $\nu \in\{n-d+2, \ldots, n\}$. Thus, it is clear that our description of $\Sigma_{d}\left(\Phi^{\prime}\right) \cap\left(\left(x_{1}, \ldots, x_{d}\right) \times Z^{\prime}\right)$ is set-theoretically correct.

10. Proof of Theorem A. We fix an integer $m \geqslant r=\operatorname{dim}(V)$ and an embedding $V \subset \mathrm{P}^{n}$ which satisfies assumption (*) of $\S 9$. Let $Z \subset \mathrm{P}^{N}$ and $\Phi: V \times$ $Z \rightarrow \mathrm{P}^{m} \times Z$ be as in Definition 8.1. Using Plücker coordinates, we obtain a dominant morphism $g: Z \rightarrow G(n, n-m-1)$. Therefore, Theorem A will follow if we can show that $\pi=\Phi_{z}$ satisfies the conclusions of Theorem A when $z$ lies in a suitable dense open subset of $Z$.

Let $\operatorname{Spec}(k) \rightarrow Z$ correspond to the closed point $z \in Z$. Then $\Sigma_{d}\left(\Phi_{z} ; q_{1}, \ldots, q_{d}\right) \cong \Sigma_{d}\left(\Phi ; q_{1}, \ldots, q_{d}\right) \times_{Z} \operatorname{Spec}(k)$. If we regard $\Sigma_{d}\left(\Phi ; q_{1}, \ldots, q_{d}\right)$ as a closed subscheme of $V \times \cdots \times V \times Z$ (product taken over $k$, as usual), this is equivalent to

$$
\Sigma_{d}\left(\Phi_{z} ; q_{1}, \ldots, q_{d}\right) \times\{z\}=\Sigma_{d}\left(\Phi ; q_{1}, \ldots, q_{d}\right) \cap(V \times \cdots \times V \times\{z\}) .
$$

By Theorem 9.1, $\operatorname{dim}\left(S_{1}^{\left(q_{*}\right)}(\Phi)\right)=r-q_{*}(m-r+1)+\operatorname{dim}(Z)<\operatorname{dim}(Z)$. Therefore $(V \times\{z\}) \cap S_{1}^{\left(q^{*}\right)}(\Phi)=\varnothing$ if $z$ lies in a suitable dense open subset of $Z$. Equivalently, we may replace $Z$ by a dense open subset and assume

(I) $S_{1}^{(q)}(\Phi)=\varnothing$ when $q \geqslant q_{*}$.

A similar argument shows that if $Z$ is replaced by a still smaller dense open subset, we may assume

(IIa) if $m>r$, then $\Sigma_{d}(\Phi)=\varnothing$ when $d \geqslant d_{*}$;

(IIb) if $m=r$, then $\Sigma_{d}(\Phi ; 1, \ldots, 1)=\varnothing$ when $d \geqslant d_{*}$.

In the case $m \geqslant r+1$ it follows that only finitely many $\Sigma_{d}\left(\Phi ; q_{1}, \ldots, q_{d}\right)$ are nonempty; if $m=r$ we conclude that only finitely many of the $\Sigma_{d}\left(\Phi ; q_{1}, \ldots, q_{d}\right)$ with $q_{j}>0$ for all $j$ are nonempty. Since the intersection of finitely many dense open subsets of $Z$ is dense, we may therefore fix $q_{1}, \ldots, q_{d}$ and consider the morphism $p: \Sigma_{d}\left(\Phi ; q_{1}, \ldots, q_{d}\right) \rightarrow Z$ induced by $V \times \cdots \times V \times Z \rightarrow Z$. Specifically, we must show that if $z$ lies in some dense open subset of $Z$, then

(i') $p^{-1}(z)$ has pure dimension $d r-(d-1) m-\Sigma_{j=1}^{d} q_{j}(m-r+1)$;

(ii') if $\operatorname{char}(k) \nmid\left(q_{j}+1\right)$ for all $j$, then $p^{-1}(z)$ is smooth over $k$;

(iii') in general, the dimension of the nonsmooth locus of $p^{-1}(z)$ is $\leqslant \operatorname{card}(Q)$ - 1, where $Q=\left\{j \mid\right.$ char $(k)$ divides $\left.q_{j}+1\right\}$.

Since $\operatorname{dim}\left(\Sigma_{d}\left(\Phi ; q_{1}, \ldots, q_{d}\right)\right)-\operatorname{dim}(Z)=d r-(d-1) m-$ $\Sigma_{j=1}^{d} q_{j}(m-r+1),\left(i^{\prime}\right)$ follows from well-known properties of the fibres of a morphism, regardless of the characteristic. If $\operatorname{char}(k)=0$, then (ii') follows 
from Theorem 2.9, and (iii') is meaningless. If $\operatorname{char}(k)>0$, then (ii') and (iii') follow from Lemma 10.3 below. Q.E.D. for Theorem A.

REMARK 10.1. Let $m=r$; suppose that $d \geqslant d_{*}$, and $\left(x_{1}, \ldots, x_{d}\right) \in$ $\Sigma_{d}\left(\Phi_{z} ; q_{1}, \ldots, q_{d}\right)$. By (IIb), we may assume that $q_{d_{*}}=\cdots=q_{d}=0$ and $x_{j}$ $\notin S_{1}\left(\Phi_{z}\right), j=d_{*}, \ldots, d$. By Proposition 5.4, smoothness of $\Sigma_{d}\left(\Phi_{z}, q_{1}, \ldots, q_{d}\right)$ at $\left(x_{1}, \ldots, x_{d}\right)$ follows from smoothness of $\Sigma_{d_{*}-1}\left(\Phi_{2} ; q_{1}, \ldots, q_{d_{*}-1}\right)$ at $\left(x_{1}, \ldots, x_{d_{*}-1}\right)$. [With $W_{j}=S_{1}^{\left(q_{j}\right)}\left(\Phi_{z}\right)$, we have $\operatorname{Ker}\left(\rho_{j}\right)=0, j=d_{*}, \ldots, d$.] This explains why it suffices to consider the values $d=1, \ldots, d_{*}-1$ when proving the case $m=r$ of Theorem A.

Definition 10.2. Let $T$ be a subscheme of $V \times \cdots \times V \times Z$ which is smooth over $k$ and of pure dimension, with $\operatorname{dim}(T) \geqslant \operatorname{dim}(Z)$. Let $p: T \rightarrow Z$ be induced by the projection $V \times \cdots \times V \times Z \rightarrow Z$. We define the nonsmooth locus of $T$ over $Z$, denoted $N S(T / Z)$, to be $S_{\nu}(p)$, where $\nu=\operatorname{dim}(T)-\operatorname{dim}(Z)+1$.

As in the proof of Theorem 2.9, we observe that $s \in T$ is a closed point of $N S(T / Z)$ if and only if either

(a) $p^{-1}(p(s)$ ) is not smooth (over $k$ ) at $s$, or

(b) $s$ is contained in an irreducible component of $p^{-1}(p(s))$ which has $\operatorname{dimension}>\operatorname{dim}(T)-\operatorname{dim}(Z)$.

LEMMA 10.3. Let $0 \leqslant d \leqslant d_{*}$ and $0 \leqslant q_{j} \leqslant q_{*}-1, j=1, \ldots, d$. Let $W=\Sigma_{d}\left(\Phi ; q_{1}, \ldots, q_{d}\right)$ be identified with a subscheme of $V \times \cdots \times V \times Z$. Then $\operatorname{dim}(N S(W / Z)) \leqslant \operatorname{dim}(Z)+\operatorname{card}(Q)-1$, where $Q=\{j \mid \operatorname{char}(k)$ divides $\left.q_{j}+1\right\}$.

The proof of Lemma 10.3 is given in the next section. Similar methods can also be used to prove the following more detailed result, of which Theorem 10.5 is an immediate consequence.

Lemma 10.4. Let $1 \leqslant e \leqslant d \leqslant d_{*}$, and let $q_{1}, \ldots, q_{d}, q_{1}^{\prime}, \ldots, q_{e}^{\prime}$ be integers such that $0 \leqslant q_{j} \leqslant q_{*}-1, j=1, \ldots, d$, and $0 \leqslant q_{j}^{\prime} \leqslant q_{j}, j=1, \ldots, e$. Let $W=\Sigma_{d}\left(\Phi ; q_{1}, \ldots, q_{d}\right), W^{\prime}=\Sigma_{e}\left(\Phi ; q_{1}^{\prime}, \ldots, q_{e}^{\prime}\right)$, and $Y=W \cap\left(N S\left(W^{\prime} / Z\right)\right.$ $\times V \times \cdots \times V)$. Then $\operatorname{dim}(Y) \leqslant \operatorname{dim}(W)-\delta-1$, where

$$
\begin{aligned}
& \delta=r-(e-1)(m-r)-\sum_{j=1}^{e} q_{j}^{\prime}(m-r+1)-\operatorname{card}(Q)-\operatorname{card}\left(Q^{\prime}\right), \\
& Q=\left\{j \mid 1 \leqslant j \leqslant e, q_{j}^{\prime}=q_{j}, \text { and } \operatorname{char}(k) \text { divides } q_{j}+1\right\}, \text { and } \\
& Q^{\prime}=\left\{j \mid 1 \leqslant j \leqslant e \text { and } q_{j}^{\prime}<q_{j}\right\} .
\end{aligned}
$$

In other words, $\left(x_{1}, \ldots, x_{d}, z\right) \in Y$ if and only if $\left(x_{1}, \ldots, x_{d}, z\right) \in W$ and $\left(x_{1}, \ldots, x_{e}, z\right) \in N S\left(W^{\prime} / Z\right)$. We obtain Lemma 10.3 by taking $e=d$ and $q_{j}^{\prime}=q_{j}, j=1, \ldots, d$. 
THEOREM 10.5. Let $d, e, q_{1}, \ldots, q_{d}$, and $q_{1}^{\prime}, \ldots, q_{e}^{\prime}$ be as above. Let $\pi: V \rightarrow \mathrm{P}^{m}$ be a generic projection, and let $\Theta \subset V \times \cdots \times V(d$-fold product $)$ consist of all $\left(x_{1}, \ldots, x_{d}\right)$ such that

(a) $\left(x_{1}, \ldots, x_{d}\right) \in \Sigma_{d}\left(\pi ; q_{1}, \ldots, q_{d}\right)$,

(b) $\left(x_{1}, \ldots, x_{e}\right)$ is a nonsmooth point of $\Sigma_{e}\left(q_{1}, \ldots, q_{e}\right)$.

Then $\operatorname{dim}(\Theta) \leqslant \operatorname{dim}\left(\Sigma_{d}\left(\pi ; q_{1}, \ldots, q_{d}\right)\right)-\delta-1$, where $\delta$ is defined as above.

Corollary 10.6. Assume, as always, that the projective embedding satisfies (*). If $\pi: V \rightarrow \mathrm{P}^{m}$ is a generic projection and $x$ is a nonsmooth point of $S_{1}^{(q)}(\pi)$, then

(i) $x \notin S_{1}^{(q+1)}(\pi)$;

(iia) $\pi^{-1}(\pi(x))=\{x\}$ if $m>r$;

(iib) $\pi^{-1}(\pi(x)) \cap S_{1}(\pi)=\{x\}$ if $m=r$.

REMARK 10.7. With the hypotheses of Theorem 10.5 , let $\left(x_{1}, \ldots, x_{d}\right) \in$ $\Sigma_{d}\left(\pi ; q_{1}, \ldots, q_{d}\right)$ and set $y=\pi\left(x_{1}\right)=\cdots=\pi\left(x_{d}\right) \in \mathrm{P}^{m}$. Set $\left(0_{y}, \mathrm{~m}_{y}\right)=$ $O_{p m}^{m}, y\left(O_{x_{j}}, m_{x_{j}}\right)=O_{V, x_{j}}$, and let $I_{j} \subset O_{x_{j}}$ be the ideal defining the subscheme $S_{1}^{\left(q_{j}^{\prime}\right)}(\pi) \subset V$ locally, $j=1, \ldots$, e. Let $N_{j}=\operatorname{Ker}\left(\rho_{j}\right) \subset \mathrm{m}_{y} / \mathrm{m}_{y}^{2}$, where $\rho_{j}: \mathrm{m}_{y} / \mathrm{m}_{y}^{2}$ $\rightarrow \mathrm{m}_{x_{j}} /\left(I_{j}+\mathrm{m}_{x_{j}}^{2}\right)$ is induced by $\pi_{j}^{*}: \mathrm{O}_{y} \rightarrow \mathrm{O}_{x_{j}}$. For simplicity, assume that $x_{j}$ $\notin S_{1}^{\left(q_{j}+1\right)}(\pi)$ if $1 \leqslant j \leqslant e$ and $q_{j}=q_{j}^{\prime}$. Lemma 11.2 below implies that $\delta=m-$ $\Sigma_{j=1}^{e} \operatorname{dim}_{k}\left(N_{j}\right)$. By Proposition 5.4 we conclude that if $\delta<0$, then $\left(x_{1}, \ldots, x_{e}\right)$ cannot be a smooth point of $\Sigma_{e}\left(\pi ; q_{1}^{\prime}, \ldots, q_{e}^{\prime}\right)$. Of course, this does not imply that $\Sigma_{e}\left(\pi ; q_{1}^{\prime}, \ldots, q_{e}^{\prime}\right)$ is empty.

11. Dimension of the nonsmooth locus. We will keep the assumptions of §9. In particular $Z \subset \mathrm{P}^{N}$ and $\Phi: V \times Z \rightarrow \mathrm{P}^{m} \times Z$ are as in Definition 8.1. As we have noted before, $\Sigma_{d}\left(\Phi_{z}\right) \times\{z\}=\Sigma_{d}(\Phi) \cap(V \times \cdots \times V \times\{z\})$. Since (*) implies condition $(\alpha)$ of $\S 7$, Theorem 7.6 implies that we may replace $Z$ with a dense open subset and assume

(A) If $0 \leqslant d \leqslant d_{*}$, then $\Sigma_{d}(\Phi)$ is smooth over $Z$ and of pure relative dimension $d r-(d-1) m$. (We regard $\Sigma_{d}(\Phi)$ as a scheme over $Z$, with $p: \Sigma_{d}(\Phi) \rightarrow$ $Z$ induced by the projection $V \times \cdots \times V \times Z \rightarrow Z$.)

Proof of Lemma 10.3. We fix $d \leqslant d_{*}$ and $q_{j}, j=1, \ldots, d$, such that $0 \leqslant q_{j} \leqslant q_{*}-1$. Let $\epsilon=\operatorname{card}\left(\left\{j \mid q_{j}>0\right\}\right)$. If $\epsilon=0$, then $q_{1}=\cdots=q_{d}=0$, and smoothness follows directly from (A). Thus, we may assume that $q_{1}>0$. If $d \geqslant 2$, we set $W_{0}=\Sigma_{d}\left(\Phi ; 0, q_{2}, \ldots, q_{d}\right)$ and $Q_{0}=Q \cap\{2, \ldots, d\}$. tion on $\epsilon$ implies

$$
\operatorname{dim}\left(N S\left(W_{0} / Z\right)\right) \leqslant \operatorname{dim}(Z)+\operatorname{card}\left(Q_{0}\right)-1 \leqslant \operatorname{dim}(Z)+\operatorname{card}(Q)-1
$$

Therefore, we may ignore irreducible components of $N S(W / Z)$ which are contained in $N S\left(W_{0} / Z\right)$. 
We now fix a closed point $\left(x_{1}, \ldots, x_{d}, z_{0}\right) \in W$ such that

(1) $W_{0}$ is smooth over $Z$ at $\left(x_{1}, \ldots, x_{d}, z_{0}\right)$. (Thus, $\left(x_{1}, \ldots, x_{d}\right)$ is a smooth point of $\Sigma_{d}\left(\Phi_{z_{0}} ; 0, q_{2}, \ldots, q_{d}\right)$.)

(If $d=1$, we simply fix a point $\left(x, z_{0}\right) \in S_{1}^{(q)}(\Phi)$.) Let $Q^{\prime}=\left\{j \mid\left(x_{j}, z_{0}\right) \in\right.$ $\left.S_{1}^{\left(q_{j}+1\right)}(\Phi)\right\}$ and $Q^{\prime \prime}=Q-Q \cap Q^{\prime}$. Let $W_{1}=\Sigma_{d}\left(\Phi ; q_{1}^{*}, \ldots, q_{d}^{*}\right)$, where $q_{j}^{*}=$ $q_{j}+1$ (resp. $q_{j}^{*}=q_{j}$ ) if $j \in Q^{\prime}$ (resp. $j \notin Q^{\prime}$ ), and let $Y=W_{1} \cap N S(W / Z)$.

I claim that $\operatorname{dim}(Y) \leqslant \operatorname{dim}(Z)+\operatorname{card}\left(Q^{\prime \prime}\right)-(m-r) \operatorname{card}\left(Q^{\prime}\right)-1$, or equivalently that $\operatorname{dim}(Y) \leqslant \operatorname{dim}\left(W_{1}\right)-\delta-1$, where

$$
\delta=r-(d-1)(m-r)-\sum_{j=1}^{d} q_{j}(m-r+1)-\operatorname{card}\left(Q^{\prime}\right)-\operatorname{card}\left(Q^{\prime \prime}\right) .
$$

This will imply the desired conclusion because $Q^{\prime \prime} \subset Q$. We may assume that $\delta \geqslant 0$; there is nothing to prove otherwise. Finally, a descending induction on $\operatorname{card}\left(Q^{\prime}\right)$ shows that we only need to study irreducible components of $Y$ which contain $\left(x_{1}, \ldots, x_{d}, z_{0}\right)$.

We will establish the claim by finding a linear subspace $\Lambda \subset \mathrm{P}^{N}$ such that the following statements hold with $Z^{\prime}=Z \cap \Lambda$.

(P1) $z_{0} \in Z^{\prime}$, and $\left(x_{1}, \ldots, x_{d}\right) \times Z^{\prime} \subset W_{1}$.

(P2) $\operatorname{dim}\left(\left(\left(x_{1}, \ldots, x_{d}\right) \times Z^{\prime}\right) \cap N S(W / Z)\right) \leqslant \operatorname{dim}(\Lambda)-\delta-1$.

[Since $W_{1}$ is smooth over $k,(\mathrm{P} 1)$ and $(\mathrm{P} 2)$ will in fact imply that $Y$ has codimension $\geqslant \delta+1$ in $W_{1}$.] Let $y=\Phi_{z_{0}}\left(x_{1}\right)=\cdots=\Phi_{z_{0}}\left(x_{d}\right) \in \mathrm{P}^{m}$. In constructing $\Lambda$ we will use homogeneous coordinate systems $\left\{T_{0}, \ldots, T_{n}\right\}$ on $\mathbf{P}^{n}$ and $\left\{S_{0}, \ldots, S_{m}\right\}$ on $\mathbf{P}^{m}$ which satisfy (2) through (6) below.

(2) $x_{1}=(1,0, \ldots, 0) \in \mathbf{P}^{n}$, and $T_{0}\left(x_{j}\right) \neq 0, j=1, \ldots, d$.

(3) $L_{z_{0}} \subset \mathbf{P}^{n}$ is the subspace $T_{0}=\cdots=T_{m}=0$, and $\Phi_{z_{0}}^{*}: \Gamma\left(\mathbf{P}^{m}, O(1)\right)$ $\rightarrow \Gamma\left(P^{n}, O(1)\right)$ satisfies $\Phi_{z_{0}}^{*}\left(S_{i}\right)=T_{i}, i=0, \ldots, m$.

Thus, $y=(1, \ldots, 0) \in \mathrm{P}^{m}$. Henceforth, we use affine coordinates $t_{1}$, $\ldots, t_{n}$ (resp. $s_{1}, \ldots, s_{m}$ ) on an affine open subset of $\mathbf{P}^{n}$ (resp. $\mathbf{P}^{m}$ ). Since $x_{1} \in S_{1}\left(\Phi_{z_{0}}\right)-S_{2}\left(\Phi_{z_{0}}\right)$, we may assume

(4) $t_{1}, \ldots, t_{r-1}, t_{m+1}$ generate the maximal ideal $m_{x_{1}} \subset 0_{x_{1}}=O_{V, x_{1}}$, and $t_{\nu} \in \mathrm{m}_{x_{1}}^{2}, v=r, \ldots, m$.

If $x \in X$ with $\left(O_{x}, \mathrm{~m}_{x}\right)=O_{X, x}$, we set $T(X)_{x}^{*}=\mathrm{m}_{x} / \mathrm{m}_{x}^{2}$, i.e., the cotangent space. $\Phi_{z_{0}}: V \rightarrow \mathrm{P}^{m}$ induces linear maps $\lambda_{z_{0}}: T\left(\mathrm{P}^{m}\right)_{y}^{*} \rightarrow T(V)_{x_{1}}^{*}$ and (if $d \geqslant$ 2) $\rho_{j, z_{0}}: T\left(\mathrm{P}^{m}\right)_{y}^{*} \rightarrow T\left(S_{1}^{\left(q_{j}\right)}\left(\Phi_{z_{0}}\right)\right)_{x_{i}}^{*}, j=2, \ldots, d$. Let $N_{1}^{0}=\operatorname{Ker}\left(\lambda_{z_{0}}\right)$ and $N_{j}=\operatorname{Ker}\left(\rho_{j, z_{0}}\right), j=2, \ldots, d$. By (4), $\left\{\bar{s}_{r}, \ldots, \bar{s}_{m}\right\}$ is a basis of $N_{1}^{0}$, where $\overline{s_{i}} \in \mathrm{m}_{y} / \mathrm{m}_{y}^{2}=T\left(\mathrm{P}^{m}\right)_{y}^{*}$ is the residue class of $s_{i}$. In the case $d \geqslant 2$, Proposition 5.4 implies that we may assume

(5) There are disjoint subsets $E_{2}, \ldots, E_{d} \subset\{1, \ldots, r-1\}$ such that $\left\{\overline{s_{i}} \mid i \in E_{j}\right\}$ is a basis of $N_{j}, j=2, \ldots, d$.

Finally, by assumption (*) of $\S 9$ we may assume 
(6) If $1 \leqslant l \leqslant q_{1}$, and $1 \leqslant \mu \leqslant r-1$, then there is an integer $\gamma(l, \mu)$ such that (a) $t_{\gamma(l, \mu)} \equiv t_{\mu} t_{m+1}^{l}\left(\bmod \mathrm{m}_{x_{1}}^{q_{1}+2}\right)$, and (b) $t_{\gamma(l, \mu)} \in \mathrm{m}_{x_{j}}^{q_{j}+2}$ if $2 \leqslant j \leqslant d$. Having obtained good coordinate systems on $\mathbf{P}^{n}$ and $\mathbf{P}^{m}$, we now fix a homogeneous coordinate system $\left\{\sigma_{i v} \mid 0 \leqslant i \leqslant m\right.$ and $\left.0 \leqslant \nu \leqslant n\right\}$ on $\mathrm{P}^{N}$ which satisfies the conclusions of Lemma 8.3. If $d=1$, we set $N=(0) \subset T\left(\mathrm{P}^{m}\right)_{y}^{*}$ and $c=0$. If $d \geqslant 2$, we set $N=N_{2}+\cdots+N_{d}$ and $c=\operatorname{dim}\left(N_{2}\right)+\cdots+\operatorname{dim}\left(N_{d}\right)$; in this case we may assume that $E_{2} \cup \cdots \cup E_{d}=\{r-c, \ldots, r-1\}$. We take $\Lambda \subset \mathrm{P}^{N}$ to be the linear subspace given by the vanishing of all $\sigma_{i \nu}-\delta_{i \nu} \sigma_{00}$ such that $(i, v) \notin \Delta$, where

$$
\begin{aligned}
& \Delta=\{(i, \nu) \mid r \leqslant i \leqslant m \text { and } \nu=\gamma(l, \mu) \text { for some }(l, \mu) \\
& \text { such that } \left.1 \leqslant l \leqslant q_{1} \text { and } 1 \leqslant \mu \leqslant r-c-1\right\} .
\end{aligned}
$$

An argument similar to the proof of Lemma 9.2 shows that $Z^{\prime}=Z \cap \Lambda$ satisfies (P1); moreover

(7) If $z \in Z^{\prime}$, then $\left(x_{j}, z\right) \in S_{1}^{\left(q_{j}+1\right)}(\Phi)$ if and only if $j \in Q^{\prime}$. For $z \in Z^{\prime}$, $\Phi_{z}: V \rightarrow \mathrm{P}^{m}$ induces linear maps $\lambda_{z}: T\left(\mathrm{P}^{m}\right)_{y}^{*} \rightarrow T(V)_{x_{1}}^{*}$ and $\rho_{j, z}: T\left(\mathrm{P}^{m}\right)_{y}^{*} \rightarrow$ $T\left(S_{1}^{\left(q_{j}\right)}\left(\Phi_{z}\right)\right)_{x_{j}}^{*}, j=2, \ldots, d$. Lemma 11.1 below implies that $\left\{\bar{s}_{i} \mid i \in E_{j}\right\}$ is a basis of $\operatorname{Ker}\left(\rho_{j, z}\right)$. Thus, Corollary 5.6 implies

(8) $\left(x_{1}, \ldots, x_{d}\right)$ is a smooth point of $\Sigma_{d}\left(\Phi_{z} ; q_{1}, \ldots, q_{d}\right)$ if and only if there exist elements $b_{i} \in I_{1}, i=1, \ldots, q_{1}(m-r+1)$, where $I_{1} \subset O_{x_{1}}=$ $O_{V, x_{1}}$ is the ideal defining $S_{1}^{\left(q_{1}\right)}\left(\Phi_{z}\right) \subset V$, such that the elements $\vec{b}_{i} \in \mathrm{m}_{x_{1}} / \mathrm{m}_{x_{1}}^{2}$, $i=1, \ldots, q_{1}(m-r+1)$, are linearly independent modulo the subspace of $\mathrm{m}_{x_{1}} / \mathrm{m}_{x_{1}}^{2}$ spanned by $\left\{\bar{t}_{i} \mid r-c \leqslant i \leqslant r-1\right\}$.

Specifically, Lemma 3.8 implies that $I_{1}$ is generated by the elements $D^{(\lambda)} \Phi_{z}^{*}\left(s_{i}\right), r \leqslant i \leqslant m$ and $1 \leqslant \lambda \leqslant q_{1}$, where the $D^{(\lambda)}: 0_{x_{1}} \rightarrow 0_{x_{1}}$ are $k$-linear differential operators such that

(9) If $b \in O_{x_{1}}$ and $b \equiv u t_{m+1}^{l}\left(\bmod m_{x_{1}}^{q_{1}+2}\right)$ where $u$ is a monomial in $t_{1}, \ldots, t_{r-1}$, then $D^{(\lambda)} b \equiv\left(\begin{array}{l}l \\ \lambda\end{array}\right) u t_{m+1}^{l-\lambda}\left(\bmod m_{x_{1}}^{2}\right)$.

Let $\Lambda_{0} \subset \Lambda$ be the complement of the hyperplane $\sigma_{00}=0$. We set $\sigma_{00}=$ 1 to obtain a set of linear coordinate functions on $\Lambda_{0}$, which we denote $\left\{\sigma_{i \nu} \mid(i, \nu)\right.$ $\in \Delta\}$. If $z \in Z^{\prime}$ and $\Phi_{z}^{*}: O_{y} \rightarrow O_{x_{1}}$ is induced by $\Phi_{z}$, then

$$
\begin{aligned}
& \Phi_{z}^{*}\left(s_{i}\right)=t_{i}, \quad i=1, \ldots, r-1, \\
& \Phi_{z}^{*}\left(s_{i}\right)=t_{i}+\sum_{\nu} \sigma_{i \nu}(z) t_{\nu}, \quad i=r, \ldots, m,
\end{aligned}
$$

where $\nu$ takes the values $\nu=\gamma(l, \mu)$, with $1 \leqslant \mu \leqslant r-c-1$ and $1 \leqslant l \leqslant q_{1}$. By (6), we may therefore assume that the following congruences hold modulo $\mathrm{m}_{x_{1}}^{q_{1}}+2$ :

$$
\Phi_{2}^{*}\left(s_{r}\right) \equiv t_{m+1}^{q_{1}+1}+\sum_{\mu=1}^{r-c-1} \sum_{l=1}^{q_{1}} \sigma_{\mu r l}^{\prime}(z) t_{\mu} t_{m+1}^{l}+\xi_{r} \quad \text { if } 1 \notin Q^{\prime} ;
$$




$$
\begin{gathered}
\Phi_{z}^{*}\left(s_{r}\right) \equiv \sum_{\mu=1}^{r-c-1} \sum_{l=1}^{q_{1}} \sigma_{\mu r l}^{\prime}(z) t_{\mu} t_{m+1}^{l}+\xi_{r} \quad \text { if } 1 \in Q^{\prime} ; \\
\Phi_{z}^{*}\left(s_{i}\right) \equiv \sum_{\mu=1}^{r-c-1} \sum_{l=1}^{q_{1}} \sigma_{\mu i l}^{\prime}(z) t_{\mu} t_{m+1}^{l}+\xi_{i}, \quad i=r+1, \ldots, m,
\end{gathered}
$$

where $\sigma_{\mu i l}^{\prime}=\sigma_{i, \gamma(l, \mu)}+a_{\mu i l}$ for some $a_{\mu i l} \in k$ [similarly for $\sigma_{\mu r l}^{\prime}$ ], and $\xi_{r}, \ldots$, $\xi_{m}$ are polynomials in $t_{1}, \ldots, t_{r-1}, t_{m+1}$ which do not involve the monomials $t_{\mu} t_{m+1}^{l}, 1 \leqslant \mu \leqslant r-c-1$, or powers of $t_{m+1}$. These congruences, together with (9), imply that the following hold modulo $m_{x_{1}}^{2}$ :

$$
\begin{aligned}
& \text { (10a) } D^{\left(q_{1}\right)} \Phi_{z}^{*}\left(s_{r}\right) \equiv\left(q_{1}+1\right) t_{m+1}+\sum_{\mu=1}^{r-c-1} \sigma_{\mu r q_{1}}^{\prime}(z) t_{\mu}+D^{\left(q_{1}\right)} \xi_{r} \quad \text { if } 1 \notin Q^{\prime} ; \\
& \text { (10b) } D^{\left(q_{1}\right)} \Phi_{z}^{*}\left(s_{r}\right) \equiv \sum_{\mu=1}^{r-c-1} \sigma_{\mu r q_{1}}^{\prime}(z) t_{\mu}+D^{\left(q_{1}\right)} \xi_{r} \quad \text { if } 1 \in Q^{\prime} ; \\
& \text { (11) } D^{(\lambda)} \Phi_{z}^{*}\left(s_{i}\right) \equiv \sum_{\mu=1}^{r-c-1} \sigma_{\mu i \lambda}^{\prime}(z) t_{\mu}+D^{(\lambda)} \xi_{i} \text { if }(i, \lambda) \neq\left(r, q_{1}\right) .
\end{aligned}
$$

The linear term of $D^{(\lambda)} \xi_{i}$ involves $t_{r-c-1}, \ldots, t_{r-1}$. By (8) it does not matter.

We now consider a matrix $M$, whose entries are to be the $\sigma_{\mu i \lambda}^{\prime}$ (distinct indeterminates, lying in the coordinate ring of $\left.\Lambda_{0}\right)$. $M$ will have $r-c-1$ columns, indexed by the integers $\mu=1, \ldots, r-c-1$. If $1 \notin Q^{\prime} \cup Q^{\prime \prime}$ (i.e., if $x_{1} \notin$ $S_{1}^{\left(q_{1}+1\right)}$ and $\left.\operatorname{char}(k) \nmid\left(q_{1}+1\right)\right)$, we take the rows of $M$ to be indexed by $\{(i, \lambda) \mid$ $r \leqslant i \leqslant m, 1 \leqslant \lambda \leqslant q_{1}$, and $(i, \lambda) \neq\left(r, q_{1}\right)$ \}. [Since $t_{m+1}$ occurs nontrivially in the linear form of $D^{\left(q_{1}\right)} \Phi_{z}^{*}\left(s_{r}\right)$, we do not need to consider the coefficients of $t_{1}, \ldots, t_{r-c-1}$ in (10a).] If $1 \in Q^{\prime}$ or if $\operatorname{char}(k) \mid\left(q_{1}+1\right), M$ will have one more row-we do not exclude $\left(r, q_{1}\right)$ from the indexing set. It follows from (8) that $\left(x_{1}, \ldots, x_{d}, z\right) \in N S(W / Z)$ if and only if $M$ has rank <\#(rows). If $d=1$, then $M$ has $r-1$ columns. If $d \geqslant 2$, then Lemma 11.2, below, and the fact that $c=$ $\operatorname{dim}\left(N_{2}\right)+\cdots+\operatorname{dim}\left(N_{d}\right)$ imply

\#(columns) $+1=r-(d-1)(m-r)-\sum_{j=2}^{d} q_{j}(m-r+1)-\operatorname{card}\left(Q_{1}^{\prime}\right)-\operatorname{card}\left(Q_{1}^{\prime \prime}\right)$, where $Q_{1}^{\prime}=Q^{\prime} \cap\{2, \ldots, d\}$ and $Q_{1}^{\prime \prime}=Q^{\prime \prime} \cap\{2, \ldots, d\}$. Therefore

$$
\# \text { (columns) }-\#(\text { rows })+1=\delta+1 \text {. }
$$

It follows that the subvariety of $\Lambda_{0}$ determined by the condition $\operatorname{rank}(M)<\#$ (rows) has codimension $\delta+1$. (See [10, Propositions 1 and 2] and [2, Theorem 3].) This implies (P2). Q.E.D.

LEMMA 11.1. With the notation of the proof of Lemma 10.3, let $z$ be an 
arbitrary point of $Z^{\prime}$. Then $T\left(S_{1}^{\left(q_{j}\right)}\left(\Phi_{z}\right)\right)_{x_{j}}$ and $T\left(S_{1}^{\left(q_{j}\right)}\left(\Phi_{z_{0}}\right)\right)_{x_{j}}$ are identical subspaces of $T(V)_{x_{j}}$, for $j=2, \ldots, d$.

PROOF. We set $x=x_{j}$ to simplify notation. $\Phi_{z}^{*}$ and $\Phi_{z_{0}}^{*}$ induce $O_{y}$-algebra structures on $O_{x}$. In each case, we get a surjective $O_{x}$-algebra homomorphism $u: P_{Q_{x} / k} \rightarrow P_{0_{x} / 0_{y}}^{q}$, where $q=q_{j}$. The two kernels are generated by $\left\{d^{q} \Phi_{z}^{*}(a) \mid\right.$ $\left.a \in \mathcal{O}_{y}\right\}$ and $\left\{d^{q} \Phi_{z_{0}}^{*}(a) \mid a \in O_{y}\right\}$ respectively. Since $\Phi_{z}(a) \equiv \Phi_{z_{0}}(a)\left(\bmod \mathrm{m}_{x}^{q+2}\right)$ and $d^{q}$ is a differential operator of order $q$, we conclude that $d^{q} \Phi_{z_{0}}^{*}(a) \equiv$ $d^{q} \Phi_{z}^{*}(a)\left(\bmod \mathrm{m}_{x}^{2} P q_{x} / k\right)$. Using a free basis of $P q_{x} / k$ to compute the two Fitting ideals, we see that corresponding generators of the Fitting ideals are congruent modulo $\mathrm{m}_{x}^{2}$. Q.E.D.

LEMMA 11.2. Let $f: X^{r} \rightarrow Y^{m}$ be a morphism of nonsingular varieties, where $r \leqslant m$. Let $q \geqslant 0$, let $x$ be a closed point of $S=S_{1}^{(q)}(f)$, and let $g=\left.f\right|_{S}$. Assume that $S$ is smooth at $x$ and locally of codimension $q(m-r+1)$ in $X$. Then the linear map $(d g)_{x}: T(S)_{x} \rightarrow T(Y)_{f(x)}$ satisfies

$$
\begin{aligned}
& \operatorname{rank}\left((d g)_{x}\right)=r-q(m-r+1) \quad \text { if } x \notin S_{1}^{(q+1)}(f) \text { and } \operatorname{char}(k) \nmid(q+1), \\
& \operatorname{rank}\left((d g)_{x}\right)=r-q(m-r+1)-1 \text { otherwise. }
\end{aligned}
$$

Proof. Let $\left(A, \mathrm{~m}_{A}\right)=0_{Y, f(x)}$ and $\left(B, \mathrm{~m}_{B}\right)=0_{X, x}$, and let $\rho=f^{*}: A$ $\rightarrow B$ be induced by $f$. Let $\left\{s_{1}, \ldots, s_{m}\right\} \subset \mathrm{m}_{A},\left\{t_{1}, \ldots, t_{r}\right\} \subset \mathrm{m}_{B}$ and the differential operators $D^{(1)}, \ldots, D^{(q)}$ be as in Lemma 3.8. The cokernel of the linear map $(d f)_{x}^{*}: T(Y)_{f(x)}^{*} \rightarrow T(X)_{x}^{*}$ is just $\mathrm{m}_{B} /\left(\mathrm{m}_{A} B+\mathrm{m}_{B}^{2}\right)$. Therefore, we must show that it is possible to find a pair $(i, l)$ such that $D^{(i)} \rho\left(u_{l}\right) \notin \mathrm{m}_{A} B+$ $\mathrm{m}_{B}^{2}$ if and only if (i) $x \notin S_{1}^{(q+1)}(f)$ and (ii) $\operatorname{char}(k) \nmid(q+1)$.

Let $\rho\left(u_{r}\right), \ldots, \rho\left(u_{m}\right)$ be expanded (modulo $m_{B}^{q+2}$ ) as power series in $t_{1}, \ldots, t_{r}$. Then $x \notin S_{1}^{(q+1)}(f)$ if and only if the monomial $t_{r}^{q+1}$ occurs in one of these expansions. By Lemma 3.7, $D_{1}^{(q)}\left(t_{r}^{q+1}\right) \equiv(q+1) t_{r}\left(\bmod \mathrm{m}_{B}^{2}\right)$, while $D^{(i)} t_{r}^{\nu} \in \mathrm{m}_{B}^{2}$ if $i \leqslant q$ and $\nu>q+1$. Therefore, $D^{(i)}\left(\rho\left(u_{l}\right)\right) \notin \mathrm{m}_{A} B+\mathrm{m}_{B}^{2}$ for some $(i, l)$ if and only if (i) and (ii) hold. [Observe that $\mathrm{m}_{B} /\left(\mathrm{m}_{A} B+\mathrm{m}_{B}^{2}\right)$ is spanned by the residue class of $t_{r}$.]

12. Further consequences of smoothness. The main result of this section is Theorem 12.1. We will prove that Theorem B is a direct consequence of Theorem 12.1.

Consider a finite morphism $f: X^{r} \rightarrow Y^{m}$ of nonsingular varieties over $k(m \geqslant r)$ and distinct closed points $x_{1}, \ldots, x_{d} \in X$ such that $f\left(x_{1}\right)=\cdots=f\left(x_{d}\right)=y \in Y$. We fix nonnegative integers $q_{1}, \ldots, q_{d}$ and assume

$$
\begin{aligned}
& \text { (1) } x_{j} \in S_{1}^{\left(q_{j}\right)}(f)-S_{1}^{\left(q_{j}+1\right)}(f), j=1, \ldots, d \text {; } \\
& \text { (2) } S_{1}^{\left(q_{j}\right)}(f) \text { is smooth and of dimension } r-q_{i}(m-r+1) \text { near } x_{j} \text {; } \\
& \text { (3) } S_{1}^{\left(q_{1}\right)}(f) \times_{Y} \cdots \times_{Y} S_{1}^{\left(q_{d}\right)}(f) \text { is smooth and of dimension } d r-(d-1) m \\
& -\Sigma_{j=1}^{d} q_{j}(m-r+1) \text { near }\left(x_{1}, \ldots, x_{d}\right) \text {. }
\end{aligned}
$$


(Of course, (3) implies (2); we list (2) only to isolate its use more clearly.) We have homomorphisms $f_{j}^{*}: \hat{O}_{Y, y} \rightarrow \hat{O}_{X, x_{j}}, j=1, \ldots, d$, induced by $f$. Let $f^{*}: \hat{0}_{Y, y} \rightarrow \Pi_{j=1}^{d} \hat{\delta}_{X, x_{j}}$, where $f^{*}(a)=\left(f_{1}^{*}(a), \ldots, f_{d}^{*}(a)\right)$. We want to identify the various complete local rings with formal power series rings in a way that will give a canonical form for $f^{*}$.

In this paragraph, we fix a value of $j$ and set $q=q_{j}$. Let $\left(A, \mathrm{~m}_{A}\right)=$ $k\left[\left[u_{1}, \ldots, u_{m}\right]\right]$, and let $\left(B_{j}, \mathrm{~m}_{j}\right)$ be a copy of $k\left[\left[t_{1}, \ldots, t_{r}\right]\right]$. By Lemma 4.5, there are isomorphisms $\phi: \hat{\mathcal{O}}_{Y, y} \stackrel{\approx}{\longrightarrow} A$ and $\psi_{j}: \hat{\mathcal{O}}_{X, x_{j}} \stackrel{\approx}{\longrightarrow} B_{j}, j=1, \ldots$, $d$, such that $g_{j}=\psi_{j} \circ f_{j}^{*} \circ \phi^{-1}: A \rightarrow B_{j}$ satisfies

(4) $g_{j}\left(u_{i}\right)=t_{i}, i=1, \ldots, r-1$;

$$
\begin{aligned}
g_{j}\left(u_{r}\right) & =t_{r}^{q+1}+\sum_{\nu=1}^{q-1} t_{q(m-r)+\nu} t_{r}^{\nu} \quad \text { if } \operatorname{char}(k) \nmid(q+1), \\
& =t_{r}^{q+1}+\sum_{\nu=1}^{q} t_{q(m-r)+\nu} t_{r}^{\nu} \quad \text { if } \operatorname{char}(k) \mid(q+1)
\end{aligned}
$$

(6) $g_{j}\left(u_{r+i}\right)=\Sigma_{\nu=1}^{q} t_{q(i-1)+\nu} t_{r}^{\nu}, i=1, \ldots, m-r$.

(If $q=0$, we are to take $g_{j}\left(u_{r}\right)=t_{r}$ and $g_{j}\left(u_{r+i}\right)=0, i=1, \ldots, m-r$.) Let $I_{j}=\Delta_{q}\left(P q_{j} / A\right)$ [with $q=q_{j}$ ] and $N_{j}=\operatorname{Ker}\left(\mathrm{m}_{A} / \mathrm{m}_{A}^{2} \rightarrow \mathrm{m}_{j} /\left(\mathrm{m}_{j}^{2}+I_{j}\right)\right)$.

Since each $I_{j}$ is the "extension to $B$ " of the ideal defining $S_{1}^{\left(q_{j}\right)}(f)$ near $x_{j}$, assumption (3) implies

(7) $\operatorname{dim}\left(N_{1}+\cdots+N_{d}\right)=\operatorname{dim}\left(N_{1}\right)+\cdots+\operatorname{dim}\left(N_{d}\right)$.

(See Proposition 5.4.) For $j=1, \ldots, d$, there are generating sets $\left\{t_{j 1}, \ldots, t_{j r}\right\}$ $\subset \mathrm{m}_{j}$ and $\left\{s_{j 1}, \ldots, s_{j m}\right\} \subset \mathrm{m}_{A}$ such that (4), (5), and (6) hold with $u_{i}=s_{j i}$, $t_{\nu}=t_{j \nu}$ and $q=q_{j}$. By Proposition 4.10, there is a subset $E_{j} \subset\left\{s_{j 1}, \ldots, s_{j m}\right\}$ such that $\left\{\bar{s}_{j i} \mid s_{j i} \in E_{j}\right\}$ is a basis of $N_{j} \subset \mathrm{m}_{A} / \mathrm{m}_{A}^{2}$. Specifically

$$
\begin{aligned}
& E_{j}=\left\{s_{j 1}, \ldots, s_{j, q(m-r+1)-1}, s_{j r}, \ldots, s_{j m}\right\} \quad \text { if } \operatorname{char}(k) \nmid\left(q_{j}+1\right), \\
& E_{j}=\left\{s_{j 1}, \ldots, s_{j, q(m-r+1)}, s_{j r}, \ldots, s_{j m}\right\} \quad \text { if } \operatorname{char}(k) \mid\left(q_{j}+1\right) .
\end{aligned}
$$

By (7), $E_{*}=E_{1} \cup \cdots \cup E_{d}$ is a subset of a minimal generating set $E=$ $\left\{s_{1}, \ldots, s_{m}\right\}$ of $m_{A}$.

It is easily seen that $g_{j}\left(E-E_{j}\right) \subset \mathrm{m}_{j}$ is a set of elements whose residues are linearly independent modulo $\left(\mathrm{m}_{j}^{2}+I_{j}\right)$. For each value of $j$, we can change $\left\{t_{j 1}, \ldots, t_{j m}\right\}$ by replacing those $t_{\nu}=t_{j \nu}$ not appearing on the right-hand sides of (5) and (6) with suitable elements of $g_{j}\left(E-E_{j}\right)$. If this is done, then (4), (5), and (6) will hold for all $j$ if $u_{1}, \ldots, u_{m}$ is taken to be a rearrangement of $s_{1}$, $\ldots, s_{m}$. The following theorem summarizes our conclusions.

THEOREM 12.1. Let $f: X^{r} \rightarrow Y^{m}$ be as above, and assume that (1), (2), and (3) hold. Then there exist

(a) isomorphisms $\psi: \hat{O}_{Y, y} \stackrel{\approx}{\longrightarrow} A=k\left[\left[s_{1}, \ldots, s_{m}\right]\right]$ and $\phi_{j}: \hat{O}_{X, x_{j}} \stackrel{\approx}{\longrightarrow}$ $B=k\left[\left[t_{1}, \ldots, t_{r}\right]\right], j=1, \ldots, d$, 
(b) permutations $\sigma_{1}, \ldots, \sigma_{d}$ of $\{1, \ldots, m\}$, and

(c) disjoint subsets $E_{1}, \ldots, E_{d}$ of $\left\{s_{1}, \ldots, s_{m}\right\}$, having the properties:

(i) The homomorphisms $g_{j}=\phi_{j} \circ f_{j}^{*} \circ \psi^{-1}: A \rightarrow B, j=1, \ldots$, $d$, satisfy (4), (5), and (6) with $g=g_{j}, q=q_{j}$, and $u_{i}=s_{\sigma_{j}(i)}, i=1, \ldots, m$;

(ii) the subsets $E_{j}, j=1, \ldots, d$, satisfy (8) with $s_{j i}=s_{\sigma_{j}(i)}$.

Proof OF THEOREM B. By Theorem 12.1 there are isomorphisms $\psi^{\prime}$ :

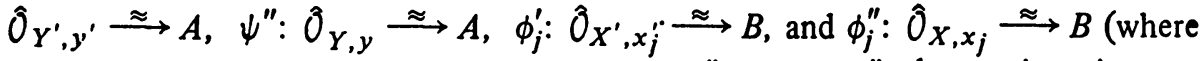
$1 \leqslant j \leqslant d$ ) such that the homomorphisms $g_{j}=\phi_{j}^{\prime \prime} \circ f_{j}^{*} \circ\left(\psi^{\prime \prime}\right)^{-1}$ and $g_{j}^{\prime}=\phi_{j}^{\prime}$ $\circ f_{j}^{*} \circ\left(\psi^{\prime}\right)^{-1}$ both satisfy (4), (5), and (6). Changing $\psi^{\prime}$ by an automorphism which permutes the variables, we may assume that $g_{j}=g_{j}^{\prime}$ for all $j$. Let $\psi=$ $\left(\psi^{\prime}\right)^{-1} \circ \psi^{\prime \prime}$ and $\phi_{j}=\left(\phi_{j}^{\prime}\right)^{-1} \circ \phi^{\prime \prime}$. Then $f_{j}^{\prime *}=\phi_{j}^{\circ} f_{j}^{*} \circ \psi^{-1}, j=1, \ldots, d$. Q.E.D.

13. Application to generic projections. Let $m \geqslant r+1$, and let $\pi: V^{r} \rightarrow$ $\mathrm{P}^{m}$ be a generic projection, starting from a projective embedding of $V$ which satisfies assumption (*) of $\S 9$. (We will regard as generic those projections which satisfy the conclusions of Theorem A and Proposition 7.4 and have the property that $\pi: V \rightarrow V^{\prime}=\pi(V) \subset \mathrm{P}^{m}$ is finite and birational.)

Let $y \in V^{\prime}$ and $\pi^{-1}(y)=\left\{x_{1}, \ldots, x_{d}\right\} \subset V$. Then

$$
\hat{0}_{V^{\prime}, y} \cong \hat{0}_{\mathrm{P}^{m}, y} / \operatorname{Ker}\left(\pi^{*}\right) \cong \operatorname{Im}\left(\pi^{*}\right) \subset \prod_{j=1}^{d} \hat{O}_{V, x_{j}},
$$

where $\pi^{*}: \hat{0}_{\mathrm{p} m, y} \rightarrow \Pi_{j=1}^{d} \hat{0}_{V, x_{j}}$ is induced by $\pi$. Assume now that

(a) $S_{2}(\pi) \cap \pi^{-1}(y)=\varnothing$;

(b) $x_{j} \in S_{1}^{\left(q_{j}\right)}(\pi)-S_{1}^{\left(q_{j}+1\right)}(\pi)$ for $j=1, \ldots, d$;

(c) $\left(x_{1}, \ldots, x_{d}\right)$ is a smooth point of $\Sigma_{d}\left(\pi ; q_{1}, \ldots, q_{d}\right)$. [If $\operatorname{char}(k) \nmid\left(q_{j}+1\right), j=1, \ldots, d$, then smoothness is automatic in (c) since $\pi$ is generic.] The hypotheses of Theorem 12.1 are thus satisfied with $X=V$ and $Y=\mathbf{P}^{m}$. It follows that $\operatorname{Im}\left(\pi^{*}\right)$ can be explicitly described as being isomorphic to a certain subring of the direct product of $d$ copies of $k\left[\left[t_{1}, \ldots, t_{r}\right]\right]$.

(13.1) The case $m=r+1$. Instead of using the general approach outlined above, we can consider the ring homomorphisms $\pi_{j}^{*}: \hat{O}_{p^{m}, y} \rightarrow \hat{O}_{V, x_{j}}$ induced by $\pi$. Let $P_{j}=\operatorname{Ker}\left(\pi_{j}^{*}\right) \subset \hat{O}_{\mathrm{p} m, y} \cong k\left[\left[X_{1}, \ldots, X_{r+1}\right]\right]$. Then $P_{1}, \ldots, P_{d}$ are principal prime ideals, and $\operatorname{Ker}\left(\pi^{*}\right)=P_{1} \cap \cdots \cap P_{d}$. Since $\hat{O}_{V^{\prime}, y}=$ $\hat{O}_{\mathrm{p}^{m}, y} / \operatorname{Ker}\left(\pi^{*}\right)$, we conclude that $\hat{O}_{V^{\prime}, y} \cong k\left[\left[X_{1}, \ldots, X_{r+1}\right]\right] /\left(f_{1} \cdots f_{d}\right)$, where $f_{1}, \ldots, f_{d}$ are (distinct) irreducible power series.

(13.2) The case $m=r+1, r \leqslant 5$. If $m=r+1$, then $S_{2}(\pi)$ has pure dimension $r-6$. Hence $S_{2}(\pi)=\varnothing$ when $r \leqslant 5$, so that (a) holds automatically. We will now list the various possibilites for $\pi^{-1}(y)$ and the corresponding structure of $\delta_{V^{\prime}, y}$.

(0) $\pi^{-1}(y)$ consists of $d$ points of $V-S_{1}$, where $d \leqslant r+1$. This can occur 
along a subset of dimension $r-d+1$, and $\hat{\delta}_{V^{\prime}, y} \cong k\left[\left[X_{1}, \ldots, X_{r+1}\right]\right] /\left(X_{1} \cdots X_{d}\right)$.

(1a) $\pi^{-1}(y)$ consists of one point of $S_{1}-S_{1}^{(2)}$. This can occur when $r \geqslant 2$, along a subset of dimension $r-2$. If $\operatorname{char}(k) \neq 2$, then

$$
\begin{aligned}
\hat{0}_{V^{\prime}, y} & \cong k\left[\left[t_{1}, \ldots, t_{r-1}, t_{1} t_{r}, t_{r}^{2}\right]\right] \subset k\left[\left[t_{1}, \ldots, t_{r}\right]\right] \\
& \cong k\left[\left[X_{1}, \ldots, X_{r+1}\right]\right] /\left(X_{r}^{2}-X_{1}^{2} X_{r+1}\right) .
\end{aligned}
$$

If $\operatorname{char}(k)=2$ and $x$ is a smooth point of $S_{1}$, then

$$
\begin{aligned}
\hat{O}_{V^{\prime}, y} & \cong k\left[\left[t_{1}, \ldots, t_{r-1}, t_{1} t_{r}, t_{2} t_{r}+t_{r}^{2}\right]\right] \\
& \cong k\left[\left[X_{1}, \ldots, X_{r+1}\right]\right] /\left(X_{r}^{2}+X_{1}^{2} X_{r+1}+X_{1} X_{2} X_{r}\right) .
\end{aligned}
$$

REMARK. When $\operatorname{char}(k)=2$, smoothness of $S_{1}$ implies $r \geqslant 3$. Finitely many nonsmooth points of $S_{1}$ can, of course, occur. These have been studied only in the case $m=2 r-1$. (See Theorem 3 of [11].)

(1b) $\pi^{-1}(y)$ consists of one point of $S_{1}^{(2)}-S_{1}^{(3)}$. This can occur when $r \geqslant 4$, along a subset of dimension $r-4$. If $\operatorname{char}(k) \neq 3$, then

$$
\begin{aligned}
\hat{O}_{V^{\prime}, y} & \cong k\left[\left[t_{1}, \ldots, t_{r-1}, t_{1} t_{r}+t_{2} t_{r}^{2}, t_{3} t_{r}+t_{r}^{3}\right]\right] \\
& \cong k\left[\left[X_{1}, \ldots, X_{r+1}\right]\right] /\left(X_{r}^{3}+\Phi_{4}+\Phi_{5}\right),
\end{aligned}
$$

where

$$
\begin{aligned}
& \Phi_{4}=X_{1}^{2} X_{3} X_{r}-X_{1}^{3} X_{r+1}+2 X_{2} X_{3} X_{r}^{2}-3 X_{1} X_{2} X_{r} X_{r+1}, \\
& \Phi_{5}=X_{2}^{2} X_{3}^{2} X_{r}-X_{1} X_{2}^{2} X_{3} X_{r+1}-X_{2}^{3} X_{r+1}^{2}
\end{aligned}
$$

If $\operatorname{char}(k)=3$ and $x$ is a smooth point of $S_{1}^{(2)}$, then

$$
\hat{0}_{V^{\prime}, y} \cong k\left[\left[t_{1}, \ldots, t_{r-1}, t_{1} t_{r}+t_{2} t_{r}^{2}, t_{3} t_{r}+t_{4} t_{r}^{2}+t_{r}^{3}\right]\right] \text {. }
$$

(Observe that $r \geqslant 5$ when $\operatorname{char}(k)=3$ and $S_{1}^{(2)}$ is smooth.)

(2a) $\pi^{-1}(y)=\left\{x_{1}, x_{2}\right\}$, where $x_{1} \in S_{1}-S_{1}^{(2)}$ and $x_{2} \in V-S_{1}$. This can occur when $r \geqslant 3$, along a set of dimension $r-3$. If $\operatorname{char}(k) \neq 2$, then

$$
\partial_{V^{\prime}, y} \cong k\left[\left[X_{1}, \ldots, X_{r+1}\right]\right] /\left(X_{1}\left(X_{r}^{2}-X_{2}^{2} X_{r+1}\right)\right) \text {. }
$$

If $\operatorname{char}(k)=2$ and $\left(x_{1}, x_{2}\right)$ is a smooth point of $\Sigma_{2}(\pi ; 1,0)$, then

$$
\delta_{V^{\prime}, y} \cong k\left[\left[X_{1}, \ldots, X_{r+1}\right]\right] /\left(X_{1}\left(X_{r}^{2}+X_{2}^{2} X_{r+1}+X_{2} X_{3} X_{r}\right)\right) \text {. }
$$

Proof. We consider only the first isomorphism. The homomorphisms $\pi_{j}^{*}: \hat{O}_{\mathrm{P} m, y} \rightarrow \hat{O}_{V, x_{j}}, j=1,2$, can be identified with homomorphisms $g_{j}$ :

$$
\begin{aligned}
k\left[\left[X_{1}, \ldots, X_{r+1}\right]\right] \rightarrow & k\left[\left[t_{1}, \ldots, t_{r}\right]\right], j=1,2, \text { where } \\
& g_{1}\left(X_{i}\right)=t_{i}, \quad i=1, \ldots, r-1 ; \\
& g_{1}\left(X_{r}\right)=t_{2} t_{r} ; \quad g_{1}\left(X_{r+1}\right)=t_{r}^{2} ;
\end{aligned}
$$




$$
g_{2}\left(X_{i}\right)=t_{i-1}, \quad i=2, \ldots, r+1 ; \quad g_{2}\left(X_{1}\right)=0 .
$$

(In the notation of $\S 12, E_{1}=\left\{X_{2}, X_{r}, X_{r+1}\right\}$ and $E_{2}=\left\{X_{1}\right\}$; notice that these sets are disjoint.) Then $\operatorname{Ker}\left(g_{1}\right)=\left(X_{r}^{2}-X_{2}^{2} X_{r+1}\right)$ and $\operatorname{Ker}\left(g_{2}\right)=\left(X_{1}\right)$. Q.E.D.

(2b) $\pi^{-1}(y)=\left\{x_{1}, x_{2}\right\}$, where $x_{1} \in S_{1}^{(2)}-S_{1}^{(3)}$ and $x_{2} \in V-S_{1}$. This can occur when $r \geqslant 5$, along a set of dimension $r-5$. If $\operatorname{char}(k) \neq 3$, then

$$
\hat{\delta}_{V^{\prime}, y} \cong k\left[\left[X_{1}, \ldots, X_{r+1}\right]\right] /\left(X_{1}\left(X_{r}^{3}+\Psi_{4}+\Psi_{5}\right)\right) \text {, }
$$

where $\Psi_{\nu}=\Phi_{\nu}\left(X_{2}, X_{3}, X_{4}, X_{r}, X_{r+1}\right), \nu=4,5$.

(2c) $\pi^{-1}(y)$ consists of two points of $S_{1}-S_{1}^{(2)}$. This can occur when $r \geqslant$ 5 along a set of dimension $r-5$. If $\operatorname{char}(k) \neq 2$, then

$$
\hat{0}_{V^{\prime}, y} \cong k\left[\left[X_{1}, \ldots, X_{r+1}\right]\right] /\left(\left(X_{r}^{2}-X_{1}^{2} X_{r+1}\right)\left(X_{r-2}^{2}-X_{2}^{2} X_{r-1}\right)\right) \text {. }
$$

(3) $\pi^{-1}(y)=\left\{x_{1}, x_{2}, x_{3}\right\}$, where $x_{1} \in S_{1}-S_{1}^{(2)}$ and $x_{j} \in V-S_{1}, j=2$, 3. This can occur when $r \geqslant 4$ along a set of dimension $r-4$. If $\operatorname{char}(k) \neq 2$, then

$$
\hat{\delta}_{V^{\prime}, y} \cong k\left[\left[X_{1}, \ldots, X_{r+1}\right]\right] /\left(X_{1} X_{2}\left(X_{r}^{2}-X_{3}^{2} X_{r+1}\right)\right) .
$$

(4) $\pi^{-1}(y)=\left\{x_{1}, x_{2}, x_{3}, x_{4}\right\}$, where $x_{1} \in S_{1}-S_{1}^{(2)}$ and $x_{j} \in V-S_{1}$, $j=2,3,4$. This can occur when $j \geqslant 5$, along a set of dimension $r-5$. If $\operatorname{char}(k) \neq 2$, then

$$
\hat{0}_{V^{\prime}, y} \cong k\left[\left[X_{1}, \ldots, X_{r+1}\right]\right] /\left(X_{1} X_{2} X_{3}\left(X_{r}^{2}-X_{4}^{2} X_{r+1}\right)\right) .
$$

Proposition 13.3. The above list of fibres $\pi^{-1}(y)$ are the only types which occur when $m=r+1$ and $r \leqslant 5$.

Proof. Since $\Sigma_{d}(\pi)$ has pure dimension $r-d+1$, we have $\Sigma_{d}(\pi)=\varnothing$ when $d \geqslant r+2$. Thus we must have $d \leqslant r+1$ in (0). More generally, $\Sigma_{d}\left(\pi ; q_{1}, \ldots, q_{d}\right)$ has pure dimension $r-d+1-2\left(q_{1}+\cdots+q_{d}\right)$. Therefore, $\Sigma_{d}\left(\pi ; q_{1}, \ldots, q_{d}\right)$ can be nonempty only when $2\left(q_{1}+\cdots+q_{d}\right) \leqslant r-d+1$. The following table specifies which nontrivial fibres can occur.

$$
\begin{array}{cccc}
r & d & r-d+1 & \text { Values which can occur } \\
2 & 1 & 2 & q=1 \\
3 & 1 & 3 & q=1 \\
& 2 & 2 & \left(q_{1}, q_{2}\right)=(1,0) \\
4 & 1 & 4 & q=1,2 \\
& 2 & 3 & \left(q_{1}, q_{2}\right)=(1,0) \\
& 3 & 2 & \left(q_{1}, q_{2}, q_{3}\right)=(1,0,0) \\
5 & 1 & 5 & q=1,2 \\
& 2 & 4 & \left(q_{1}, q_{2}\right)=(1,0),(2,0) \text { or }(1,1) \\
3 & 3 & \left(q_{1}, q_{2}, q_{3}\right)=(1,0,0) \\
& 4 & 2 & \left(q_{1}, q_{2}, q_{3}, q_{4}\right)=(1,0,0,0)
\end{array}
$$




\section{REFERENCES}

1. J. M. Boardman, Singularities of differentiable maps, Inst. Hautes Études Sci. Publ. Math. No. 33 (1967), 21-57. MR 37 \#6945.

2. J. A. Eagon and D. G. Northcott, Ideals defined by matrices and a certain complex associated with them, Proc. Roy. Soc. London Ser. A 269 (1962), 188-204. MR 26 \#161.

3. H. Fitting, Die Determinantenideale eines Moduls, Jber. Deutsch. Math.-Verein. 46 (1936), 195-228.

4. A. Grothendieck, Éléments de géométrie algébrique. IV. Étude locale des schémas et des morphismes de schémas, $4^{\mathrm{e}}$ partie, Rédigés avec la collaboration de J. Dieudonné, Inst. Hautes Études Sci. Publ. Math. No. 32 (1967). MR 39 \#220. \#2560.

5. H. I. Levine, The singularities $S_{1}^{q}$, Illinois J. Math 8 (1964), 152-168. MR 28

6. J. Mather, Generic projections, Ann. of Math. (2) 98 (1973), 226-245.

7. B. Morin, Formes canoniques des singularités d'une application différentiable, C. $\mathbf{R}$. Acad. Sci. Paris 260 (1965), 5662-5665. MR 31 \#5212.

8. K. Mount and $O$. Villamayor, An algebraic construction of the generic singularities of Boardman-Thom, Inst. Hautes Études Sci. Publ. Math. No. 43 (1974), 205-244.

9. M. Nagata, Local rings, Interscience Tracts in Pure and Appl. Math., no. 13, Interscience, New York, 1962. MR 27 \#5790.

10. D. Northcott, Some remarks on the theory of ideals defined by matrices, Quart. J. Math. Oxford Ser. (2) 14 (1963), 193-204. MR 27 \#1467.

11. J. Roberts, Generic projections of algebraic varieties, Amer. J. Math. 93 (1971), 191-214. MR 43 \#3263.

12. The variation of singular cycles in an algebraic family of morphisms, Trans. Amer. Math. Soc. 168 (1972), 153-164: MR 46 \#5326.

13. - Singularity subschemes and generic projections, Bull. Amer. Math. Soc. 78 (1972), 706-708. MR 46 \#175.

14. - Generic coverings of $P^{r}$ when $\operatorname{char}(k)>0$, Notices Amer. Math. Soc. 20 (1973), A-383. Abstract \#704-A5.

15. S. Suzuki, Differentials of commutative rings, Queen's Papers in Pure and Appl. Math., no. 29, Queen's University, Kingston, Ont., 1971.

16. R. Thom, Les singularités des applications différentiables, Ann. Inst. Fourier (Grenoble) 6 (1955/56), 43-87. MR 19, 310.

17. H. Whitney, Singularities of mappings of Euclidean spaces, Sympos. Internac. de Topología Algebraica, Universidad Nacional Autónoma de Mexico and UNESCO, Mexico City, 1958, pp. 285-301. MR 20 \#4877.

DEPARTMENT OF MATHEMATICS, UNIVERSITY OF MINNESOTA, MINNEAPOLIS, MINNESOTA 55455 